\title{
WestVirginiaUniversity
}

THE RESEARCH REPOSITORY @ WVU

Graduate Theses, Dissertations, and Problem Reports

2019

\section{RoAdNet: Robust Adaptive Network for Information Diffusion in VANET}

Priyashraba Misra

prmisra@mix.wvu.edu

Follow this and additional works at: https://researchrepository.wvu.edu/etd

Part of the Digital Communications and Networking Commons, and the Navigation, Guidance, Control, and Dynamics Commons

\section{Recommended Citation}

Misra, Priyashraba, "RoAdNet: Robust Adaptive Network for Information Diffusion in VANET" (2019). Graduate Theses, Dissertations, and Problem Reports. 7476.

https://researchrepository.wvu.edu/etd/7476

This Dissertation is protected by copyright and/or related rights. It has been brought to you by the The Research Repository @ WVU with permission from the rights-holder(s). You are free to use this Dissertation in any way that is permitted by the copyright and related rights legislation that applies to your use. For other uses you must obtain permission from the rights-holder(s) directly, unless additional rights are indicated by a Creative Commons license in the record and/ or on the work itself. This Dissertation has been accepted for inclusion in WVU Graduate Theses, Dissertations, and Problem Reports collection by an authorized administrator of The Research Repository @ WVU.

For more information, please contact researchrepository@mail.wvu.edu. 


\title{
RoAdNet: Robust Adaptive Networks for Information Diffusion in VANETS
}

\author{
Priyashraba Misra \\ Dissertation submitted to the \\ Benjamin M. Statler College of Engineering and Mineral Resources \\ at West Virginia University \\ in partial fulfillment of the requirements \\ for the degree of \\ Doctor of Philosophy \\ in \\ Computer Engineering \\ Vinod K. Kulathumani, Ph.D., Chair \\ Matthew C. Valenti, Ph.D. \\ Yanfang Ye, Ph.D. \\ Andrew Nix, Ph.D. \\ Kakan Dey, Ph.D. \\ Lane Department of Computer Science and Electrical Engineering \\ Morgantown, West Virginia \\ 2019
}

Keywords: Vehicular Ad-Hoc Networks, V2X and V2V Communication, Vehicular Safety and Navigation

Copyright 2019 Priyashraba Misra 


\title{
Abstract \\ RoAdNet: Robust Adaptive Networks for Information Diffusion in VANETS
}

\author{
Priyashraba Misra
}

The automotive industry has changed more in the last one decade than ever before. Rapid advancements in autonomous driving have opened up opportunities for CAVs (Connected and Automated Vehicles). Vehicles today rely on a sensor-suite to map the surrounding and use that information for safety and navigation. The sensor's view is limited to its line of sight and this drawback can be tapered off by using Vehicle to Vehicle (V2V) and Vehicle to Infrastructure (V2I), generally referred to as Vehicle to Everything (V2X) communication. In this thesis, we specifically focus on utilizing V2V communication using on-board Dedicated Short Range Communication (DSRC) radios for exchanging vehicular information. Unlike V2I, this mode does not rely on the existence of densely deployed communication infrastructure but nevertheless can utilize those when available.

A significant amount of existing research in V2V using DSRC radios has focused on a single hop broadcast mode, i.e., utilize information that is within direct communication range of each other. While this is useful for safety applications like collision warnings/avoidance and lane change warnings, there are applications that benefit from transmitting information beyond a single hop communication range. For instance, V2V information can be used for optimizing vehicle routes, improving fuel efficiency and highway merging to name a few. However, transmitting information over such long distances need multi-hop communication protocols, which are challenging to design because now the available channel bandwidth is shared between single hop source transmission and forwarding of messages from multihop. As vehicular density increases, and information is disseminated over long distances, the available bandwidth for disseminating single hop messages diminishes thereby reducing the precision of information about nearby vehicles.

To address this problem, this dissertation presents RoAdNet, a multi-hop, multi-resolution protocol that draws inspiration from distance sensitive multi-hop forwarding techniques to better utilize the bandwidth and successfully send information over long distances. RoAdNet trades off information precision with distance and is thereby able to deliver information over larger distances without affecting the precision of single hop information. The protocol is evaluated using a simulation framework that emulates realistic traffic conditions. The quality of the disseminated data is evaluated as a function of distance under different traffic and communication network parameters. RoAdNet is hardware platform agnostic and seamlessly interfaces into any underlying radio technology. 


\section{Acknowledgements}

First, I want to thank my committee chair and advisor, Dr. Vinod K. Kulathumani, for guiding me in my research and providing me the opportunity to work with him and his other graduate students. This dissertation has been made possible with his constant support and guidance.

I also want to thank Dr. Matthew Valenti, Dr. Yanfang Ye, Dr. Andrew Nix and Dr. Kakan Day for being a part of my doctoral committee, and for their valuable time and advice.

I extend my gratitude towards the West Virginia University's EcoCAR3 and EcoCAR Mobility Challenge Teams and especially towards Dr. Andrew Nix for allowing me to lead the Advanced Driver Assistance Systems (ADAS) and Connected and Automated Vehicles (CAVs) sub teams. Working on this project has been a great learning experience.

I would like to thank my current and past co-workers for helping me out in understanding and troubleshooting multiple aspects of this work. They have been extremely helpful, supportive in building my understanding of the subject. I've learned from discussions with them.

I also extend my special thanks to my friends who kept me sane and motivated over the last few years.

Last but not least, I want to express my gratitude to my family. My parents have been very encouraging on my decision to go to grad school. Their support has been relentless and a constant motivation to my desire of pursuing Ph.D. in Computer Engineering at WVU. 


\section{Contents}

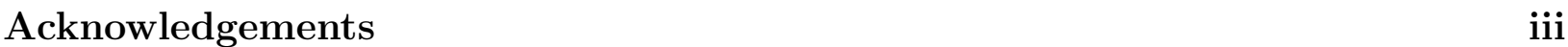

List of Figures $\quad$ vii

List of Tables $\quad$ ix

1 Introduction $\quad 1$

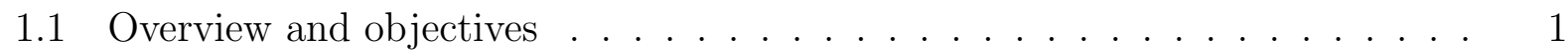

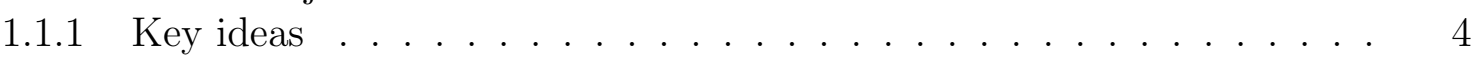

1.1.2 Performance metrics . . . . . . . . . . . . . . . 6

1.1.3 Summary of contributions . . . . . . . . . . . . . . 8

1.1.4 Outline of the Thesis . . . . . . . . . . . . . . . . 8

2 Background work and existing literature $\quad 10$

2.1 Structure-based Protocols . . . . . . . . . . . . . . . . . . . . 11

2.1.1 Directed Diffusion . . . . . . . . . . . . . . . . . . . . . . . . . . . . . . 11

2.1 .2 Tiny AGgregation . . . . . . . . . . . . . . . . 11

2.1.3 Collection Tree Protocol - CTP . . . . . . . . . . . . . . . . . 12

2.2 Beaconing . . . . . . . . . . . . . . . . . . . . 13

2.3 Structureless Protocols . . . . . . . . . . . . . . . . . . . . . . 14

2.3.1 Flooding and Gossiping . . . . . . . . . . . . . . . . . . . . 14

2.3 .2 Random Walks . . . . . . . . . . . . . . . . . . . . 14

2.4 Vehicular Communication and Applications . . . . . . . . . . . . 15

2.4.1 DSRC Based Protocols for Vehicular Communication . . . . . . . . . 16

2.4.2 Cellular Based Vehicular Communication . . . . . . . . . . . . . . 17

2.4.3 Applications of V2V Communication . . . . . . . . . . . . . . . . . 17

2.5 Adaptive Cruise Control \& Car Following Models . . . . . . . . . . . . . . . 19

3 RoAdNet Protocol Design $\quad 21$

3.1 Transmit Engine . . . . . . . . . . . . . . . . . . . . . 21

3.2 Receive Engine . . . . . . . . . . . . . . . . . . . . . . . 23

3.3 Forward Engine . . . . . . . . . . . . . . . . . . . 25

3.3.1 Traditional Forwarding Engine . . . . . . . . . . . . . . . . 25

3.3.2 Distance Sensitive Forward Engine . . . . . . . . . . . . . . . 26

3.4 Aggregate and Adapt Engine . . . . . . . . . . . . . . . . . 27 
3.5 Division of Cells for Aggregation . . . . . . . . . . . . . . . . . . . . . . . 29

3.6 Handling Packet Collision and Network Throughput . . . . . . . . . . . . . 30

3.6.1 Randomized Transmission Schedules . . . . . . . . . . . . . . . . 30

3.6.2 Multiple Packet Suppression . . . . . . . . . . . . . . . . . . . . 30

3.6.3 Distance Sensitive Forwarding . . . . . . . . . . . . . . . . . . . 31

3.7 RoAdNet Algorithm . . . . . . . . . . . . . . . . . . . . 31

4 Experimental Setup in VENTOS 33

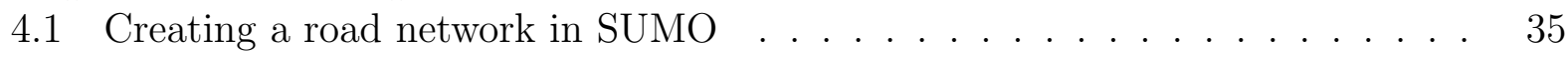

4.1 .1 Creating network file . . . . . . . . . . . . . . 35

4.1.2 Generating traffic demand . . . . . . . . . . . . . . 36

4.2 V2V Communication in VENTOS . . . . . . . . . . . . . . . . . . 38

4.3 Scenario Design for Testing RoAdNet . . . . . . . . . . . . . . . . . . 39

5 Results 41

5.1 Distance Sensitive vs Non Distance Sensitive Forwarding . . . . . . . . . . . 42

5.1 .1 Effect on Staleness . . . . . . . . . . . . . . . . . . 42

5.1 .2 Effect on Distance Estimation Error . . . . . . . . . . . . . . . 44

5.1.3 Effect on Packet Delivery Ratio . . . . . . . . . . . . . . . . . . . 44

5.1 .4 Discussion . . . . . . . . . . . . . . . . . . . 45

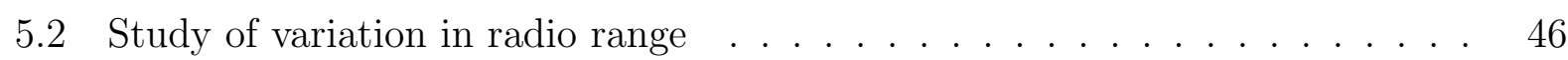

5.2 .1 Effect on Staleness . . . . . . . . . . . . . . . . . . . . . . 47

5.2.2 Effect on distance error estimation . . . . . . . . . . . . . . . . . . . . . . 48

5.2 .3 Effect on packet delivery ratio . . . . . . . . . . . . . . . 48

5.2.4 Effect on number of packets delivered . . . . . . . . . . . . . . . . . 48

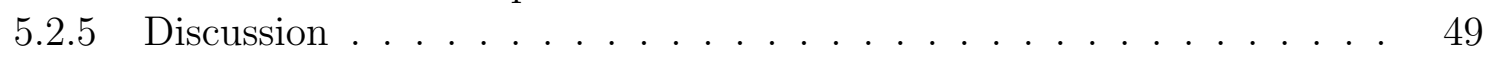

5.3 Parameter optimization in RoAdNet . . . . . . . . . . . . . . 50

5.3.1 Suppression Threshold . . . . . . . . . . . . . . . 50

5.3.2 Transmission Queue Length . . . . . . . . . . . . . . . . . 51

5.4 Effect of multi-resolution transmission . . . . . . . . . . . . . . 53

5.5 RoAdNet Overall Performance Evaluation . . . . . . . . . . . . . . . . . 55

5.5.1 Effect of Distance Sensitive Forwarding . . . . . . . . . . . . . . . 55

5.5.2 Effect of Aggregation and Forward Packets on Base Transmission . . 56

5.5.3 Latency in Aggregate Packets . . . . . . . . . . . . . . . . . . 57

5.6 A real-world test case for improving efficiency by using prior knowledge of upcoming driving scenarios . . . . . . . . . . . . . . . . . . . . . . . . . . . 58

5.6 .1 Experiment Setup . . . . . . . . . . . . . . . . . . 59

5.6 .2 Test Cases . . . . . . . . . . . . . . . . . . 61

5.6.3 Results: Regenerative Energy Captured for Different Scenarios . . . . 63

5.6 .4 Better fuel efficiency with RoAdNet . . . . . . . . . . . . . . . 65

6 Conclusion and Future Work $\quad 66$

6.1 Conclusion . . . . . . . . . . . . . . . . . . 66

6.2 Future Work . . . . . . . . . . . . . . . . . . . . 67 
$\begin{array}{ll}\text { A List of Abbreviations } & 68\end{array}$

$\begin{array}{ll}\text { B List of Symbols } & 69\end{array}$

$\begin{array}{ll}\text { References } & 70\end{array}$ 


\section{List of Figures}

1.1 RoAdNet Multi-Resolution transmissions . . . . . . . . . . . . . . . . . 5

3.1 Modules in RoAdNet . . . . . . . . . . . . . . . . . . . . . . . . 22

3.2 Position and radio range of vehicles . . . . . . . . . . . . . . . . 27

3.3 Transmission sequence of packets in traditional forwarding . . . . . . . . . . 28

3.4 Transmission sequence of packets in Distance Sensitive Forwarding . . . . . . 28

3.5 Cell design for RoAdNet Evaluation . . . . . . . . . . . . . . . . . . 31

4.1 VENTOS Architecture[1] . . . . . . . . . . . . . . . . . . . 34

4.2 Methods of generating SUMO network file . . . . . . . . . . . . . . . . . . . . . . . . . .

4.3 Four-way junctions designed in SUMO . . . . . . . . . . . . . . . . . . . . . . . . . . . . . . .

4.4 A junction with two vehicle flows . . . . . . . . . . . . . 38

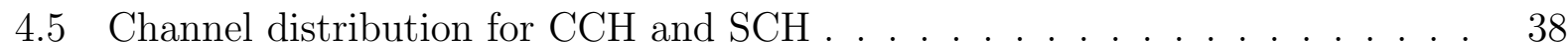

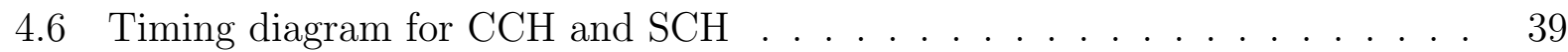

5.1 Effect of distance sensitivity on staleness for different densities . . . . . . . . 44

5.2 Effect of distance sensitivity on Distance Estimation Error for different densities 46

5.3 Effect of distance sensitivity on Packet Delivery Ratio . . . . . . . . . . . . . 47

5.4 Effect of radio range on staleness . . . . . . . . . . . . . . . . . . . . . . . . . . . . . . . . . . . .

5.5 Effect of radio range on distance estimation error . . . . . . . . . . . . . . . 49

5.6 Effect of radio range on packet delivery ratio . . . . . . . . . . . . . . 50

5.7 Effect of radio range on packets delivered per bin . . . . . . . . . . . . . 51

5.8 Effect of suppression threshold on packet delivery ratio . . . . . . . . . . . 52

5.9 Effect of suppression threshold on staleness . . . . . . . . . . . . . . . . . . . . . . . . . . . 52

5.10 Effect of queue length on packet delivery ratio . . . . . . . . . . . . . 53

5.11 Effect of queue length on staleness . . . . . . . . . . . . . . . . . 54

5.12 Packets delivery count at long distances . . . . . . . . . . . . . . . . . 54

5.13 Effect of multi-resolution forwarding on distance estimation error . . . . . . 55

5.14 Effect of distance sensitive forwarding on staleness of messages within single

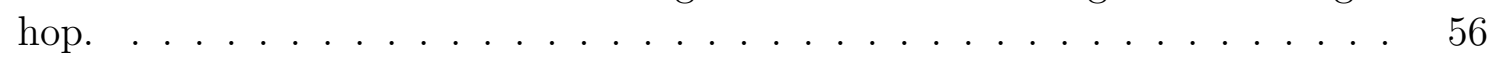

5.15 Effect of distance sensitive forwarding on staleness of messages within single

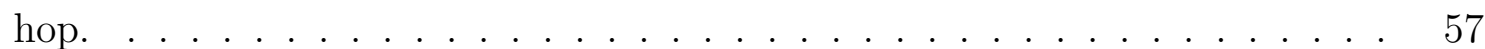

5.16 Latency of long range messages in RoAdNet . . . . . . . . . . . . . . . . 58

5.17 EcoCAR Hardware Architecture . . . . . . . . . . . . . . . . . . . 60

5.18 EcoCAR Software Architecture . . . . . . . . . . . . . . . . . . 61 


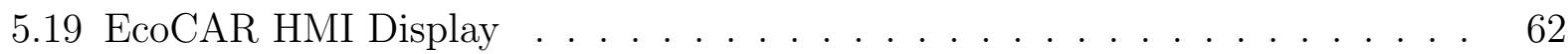

5.20 EcoCAR HMI mounted in the car . . . . . . . . . . . . . . . . . 63

5.21 The band practice field / Test site . . . . . . . . . . . . . . . 63

5.22 Energy captured using mechanical brakes only . . . . . . . . . . . . . . . 64

5.23 Energy captured using generative braking and driver feedback on HMI . . . 64 


\section{List of Tables}

3.1 Contents of data packet . . . . . . . . . . . . . . . . . . 23

3.2 Contents of Aggrecation/Cell State packet . . . . . . . . . . . . . . . . . 29

5.1 Network performance for different thresholds . . . . . . . . . . . . . . . . 50

5.2 Average energy captured . . . . . . . . . . . . . . . . . . 65 


\section{Chapter 1}

\section{Introduction}

\section{$1.1 \quad$ Overview and objectives}

Since the early $20^{\text {th }}$ century, automobiles have been commercially available and have been a big part of a country's economy and people's comfort. Over the decades automobiles have evolved from being slower and less reliable to being faster, more exciting and technology savvy. While most of the research, development and evolution of automotive technology in the $20^{\text {th }}$ century was about improving engine efficiency, and adding subtle features like electric windows, CD players, digital displays etc., the $21^{\text {st }}$ century has thus far seen significant progress towards autonomous driving. The Society of Automotive Engineers (SAE) has defined six levels of driving automation, Level 0 being no automation to Level 5 being full automation. Features like Automated Braking System (ABS), Adaptive Cruise Control (ACC) and Lane Keep Assist (LKS) are deployed in a wide variety of vehicles targeted towards Level 2 and Level 3 autonomy.

The increase in autonomous driving features, also known as Advanced Driver Assistance Systems or ADAS, have opened opportunities for research in Connected and Automated Vehicles (CAVs). While automated vehicle technology has long been researched, Connected Vehicles is still in its early stages. Connected Vehicles refers to the ability of a vehicle to communicate information to other vehicles and infrastructure with the help of Dedicated Short-Range Communication (DSRC) radios. A cluster of such vehicles with DSRC radios is referred to as Vehicular Ad-hoc Network or VANET. 
VANETS are a special category of Mobile Ad-Hoc Networks (MANETs), where the nodes under consideration are vehicles. The nodes in a MANET are capable of communicating with all nodes within their individual radio communication range, which may be different for each node. The movement of nodes in MANETs are guided by an underlying mobility model. In the case of VANETs the mobility model is much more constrained, as vehicles tend to follow a certain network of roads and have well-defined control mechanisms. Wireless communication in VANETs is commonly categorized into Vehicle to Vehicle(V2V) and Vehicle to Infrastructure(V2I) mode. This dissertation focuses specifically on V2V communication. $\mathrm{V} 2 \mathrm{~V}$ communication is used to complement the increasingly rich sensor features being deployed off late in vehicles such as cameras, radars, and LIDARs. While these sensors are used to capture information directly adjacent to a vehicle, $\mathrm{V} 2 \mathrm{~V}$ communication can be used to disseminate this sensed information to a wider neighborhood which can then be consumed by different applications.

Applications of V2V communication can be broadly classified into three categories: safety, navigation efficiency, and environmental efficiency. The type and frequency of information needed for each of these applications are different. Safety applications include forward collision warnings, adaptive cruise control, blind spot warning, and lane change warnings. These applications typically require information at higher precision and high frequency. The information can be combined with the information from on-vehicle sensors, such as radars and cameras to enhance safety and driving assistance features. Navigational efficiency applications are focused on dynamic re-routing in the presence of traffic incidents. Environmental efficiency applications are typically deployed in semi-automatic scenarios such as adaptive cruise control and autonomous vehicles. They seek to utilize traffic information and information about upcoming traffic signals to optimize fuel consumption by intelligently choosing braking and acceleration parameters.

Fueled by these application scenarios, there has been significant research on V2V communication in the past decade that have resulted in numerous information routing and dissemination protocols. A detailed survey of various communication protocols and their applications can be found in Section 2. However, most of these protocols tend to focus on specific application scenarios and hence tend to be isolated and limited in their use case. As 
a result, the protocols have difficulty in co-existing when different application scenarios have different information requirements. Some examples of such scenarios are listed below.

1. Unfortunately, the scenario in Case 1 is limited in that vehicles are warned about other vehicles only when they are too close to each other, which may fail to avoid many traffic incidents or provide a timely re-route guidance in the presence of those incidents. Therefore, there is also a concurrent need for disseminating vehicular information at larger distances than a single hop range of 300-500m. This information is important for avoiding pile-up crashes, lane merges in highways, managing fuel efficiency and dynamic ridesharing. As an example consider adaptive cruise control or semi-autonomous driving. In adaptive cruise control (ACC), car following models are used to determine how vehicles follow each other on the road. The fundamental idea of a car following model is to maintain a safe distance to the leading vehicle. Today's algorithms use information of just one leading vehicle to predict speed and acceleration for the ego vehicle (the vehicle under consideration). Although they work well in freeflowing traffic, a sudden change in driving conditions is not handled very well. The control is handed back to the driver in most cases. The fact that information about vehicles down the road is not used makes the vehicle vulnerable to high-speed crash and pile-ups on highways. Information about vehicles further down the road requires multi-hop information dissemination. But once multi-hopping is introduced, the network bandwidth is now being shared for forwarding information about other vehicles along with the source broadcast defined in Case 1. If this forwarding is not managed efficiently, then it starts adversely affecting the throughput of single hop information broadcast. Thus, there is a need for maintaining high precision in a single hop range, while at the same time supporting multi-hop communication over larger distances.

2. Finally, some applications require information about very larger distances such as 8000-10000m. Examples include dynamic rerouting. Modern Routing applications like Google Maps and Waze are dependent on cellular networks for getting real-time information about streets and finding efficient routes. Using cellular networks has its own constraints. Performance of such systems decreases rapidly in regions of limited 
or no cellular service, like in tunnels and remote areas. Also, the estimation of vehicle traffic depends on the number of people using handheld devices (mobile phones or tablets) while driving, with Global Positioning System(GPS), enabled. The information travels from the device to a centralized server, where information is processed and updates sent back to the device. Dependency on users, devices and cellular network conditions can induce latency in information. By the time one is made aware of traffic congestion, it may already be too late to adjust the route. So there is a need for realtime communication of such traffic information over large distances. But the question is how does it co-exist with meeting the information needs of case 1 and case 2 ? When information is communicated over such large distances, it will consume the network bandwidth and further reduce the throughput for safety applications.

Problem statement: The goal of this dissertation is to investigate techniques that are able to support information dissemination over large distances while maintaining high data delivery rates at closer distances for safety applications.

\subsubsection{Key ideas}

\section{Multi-Resolution Transmission Model}

To address the above problem, the key idea in this dissertation is to use a multiresolution approach in information dissemination, which we call RoAdNet (RObust ADaptive NETworks)[2]. A multi-resolution transmission model works by prioritizing transmission based on how critical the information is. The quality of the information in the network is determined by the application it is being used for. To better understand how the quality of information is related to application we organize $\mathrm{V} 2 \mathrm{~V}$ and $\mathrm{V} 2 \mathrm{X}$ application in to three distinct categories based on distance and information quality.

Firstly, within a one-hop communication range, we utilize a high broadcast rate. Using a high broadcast rate ensures that information about vehicles in the vicinity are updated more frequently and hence high quality information about is available about individual vehicles. This is important because most applications within single hop range are safety critical application, like collision avoidance and lane keep assist.

Secondly, beyond this single hop range and up to a $1500 \mathrm{~m}$, we use a distanced based 
reduction in broadcast rates - this allows us to minimally impact the source broadcast rate while at the same time conveying individual vehicle information up to a $1500 \mathrm{~m}$. The quality of the information is lower as compared to the first case. This is reasonable because vehicles at larger distances have progressively more reaction time to adjust their trajectory and speed. Applications that can use such messages are highway merging, safe navigation around blind curves and avoiding high speed pile-up crashes involving multiple vehicles.

Lastly, Beyond a distance of $1.5 \mathrm{kms}$ and up to a distance of $10 \mathrm{kms}$, we provide aggregated information about cells within the region. A cell can be a street, a group of streets or a geographic region. The Aggregate information could be average estimated speed or specific traffic alerts such as the presence of accident or high congestion. This can be used by vehicles at larger distances to adjust their trajectory and avoid the region.

As seen in Fig.1.1, short range safety critical messages are transmitted at high data rates to maintain high precision in information quality. Medium range information is staggered using a distance sensitive approach, where messages from farther distances are forwarded less frequently. Long range aggregation messages are sparsely transmitted unless an event like an accident or congestion is detected.

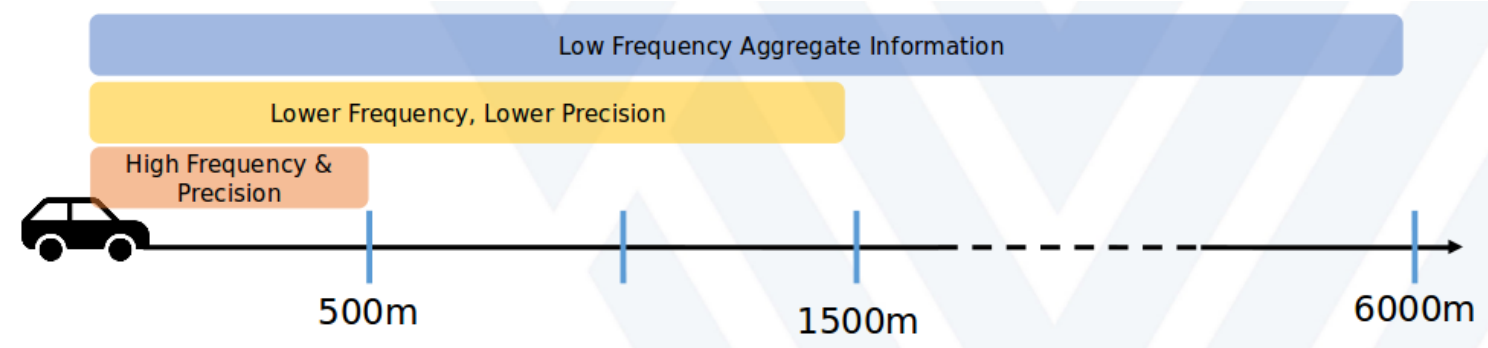

Figure 1.1: RoAdNet Multi-Resolution transmissions

This multi-resolution approach leads us to several interesting questions:

1. How to dynamically adjust the transmission range that constitutes a single hop?

2. how to seamlessly aggregate information about vehicles in a cell?

3. How does distance based reduction in forwarding rates impact accuracy?

4. What is the appropriate source broadcast rate to choose? 
5. What is the appropriate function for progressively reducing the forwarding rates based on distance?

6. How much reaction time does the aggregation strategy allow for vehicles to re-route?

This dissertation seeks to address these questions through simulations in VENTOS (VEhicular NeTwork Open Simulator), which is an integrated simulator for studying network protocols built using OMNeT++ (Objective Modular Network Test-bed ), under various traffic conditions simulated using realistic driving patterns injected via SUMO (Simulation of Urban Mobility).

\subsubsection{Performance metrics}

We measure the performance of RoAdNet based on the parameters defined below. It must be noted that the entire simulation region was divided into buckets of 100 meters each. All the information coming from a bucket was averaged to give an aggregate which would help measure the network characteristics better as compared to individual vehicle information.

\section{Staleness}

Staleness of a packet from vehicle $i$ at vehicle $j$, denoted as $S(i, j)$ is the measure of the quality of information about vehicle $j$. It tells how old the current information about a particular vehicle is or the time since a packet has been received from a particular vehicle. Staleness, as shown in equation 1.1, is calculated as the difference between the current simulation time $(t)$ and the last times when a message was heard from a vehicle $i\left(t_{i}\right)$.

$$
S(i, j)=t-t_{i}
$$

\section{Packet Deliver Ratio}

Packet delivery ratio is the ratio of the total number of packets that were received in the network to the total number of packets that were transmitted.

$$
\widehat{P D R}=\frac{n_{r x}}{n_{t x}}
$$


Equation 1.2 shows the packet delivery ratio as the ratio of total number of packets received by all the vehicles $n_{r x}$ to the total number of packets transmitted by all the vehicles $n_{t x}$.

The PDR is further normalized to better understand the network characteristics within the communication range of a single vehicle. The normalized packet delivery ratio is represented as PDR. For a given density of vehicles $(\rho)$ within the communication range the PDR is given by euation.1.3

$$
P D R=\frac{\widehat{P D R}}{\rho}
$$

\section{Distance Estimation Error Rate}

The error rate is the ratio of the error in estimated distance divided by actual distance. The receiving vehicle uses the information about the sending vehicle in the message to estimate the distance to the sending vehicle.

$$
D E E R=\frac{\sum_{i=1}^{n} \Delta}{n}
$$

The DEER for $n$ vehicles, as shown in equation 1.4 is the ratio of total error in distance estimation $(\delta)$ of all vehicles to the number of vehicles.

$$
\Delta=\frac{d-\hat{d}}{d}
$$

The error ratio in distance estimation is given by the ratio of error in distance estimation to the actual distance. In equation $1.5, d$ is the actual distance and $\hat{d}$ is the estimated distance to the vehicle.

\section{Latency}

Latency of a packet from vehicle $i$ at vehicle $j$ is defined at the delay between origin of packet at vehicle $i$ and delivery of packet at vehicle $j$. Latency will be used to characterize the performance of the Aggregate Engine. Latency will show how long it takes for a vehicle at a certain distance to know about an event. 


$$
L(i, j)=t_{r x_{-} j}-t_{t x_{-} i}
$$

Equation 1.6 shows the latency of a packet from vehicle $i$ at vehicle $j$. It is the difference between the time a packet was received at vehicle $j\left(t_{r x_{-} j}\right)$ and the time the packet was transmitted from vehicle $i\left(t_{t x_{-} i}\right)$.

\subsubsection{Summary of contributions}

- In this thesis, we describe a protocol that integrates single hop broadcast, multi-hop distance based broadcast and long distance aggregate broadcast seamless into a single multi-resolution dissemination strategy and investigates the impact of numerous parameter choices on the performance.

- The protocol parameters shall be designed for adaptation to the specific transportation application requirements and dynamic roadway traffic conditions in terms of vehicular density and speed to maximize the information throughput and robustness of the system.

- We implement the protocol in the VEhiculat NeTwork Open Simulator(VENTOS), which allows wireless protocols modeled in OmNet ++ to interact with mobility models generated by Simulation of Urban Mobility(SUMO).

- We study the impact of radio range, base transmission frequency and distance sensitive forwarding on Staleness, Packet Delivery Ratio, and Distance Estimation Error Ratio.

- The protocol developed in this thesis is expected to be radio platform agnostic, thus permitting their seamless interface into any underlying V2V radio technology such as Wi-Fi, DSRC, or C-V2X

\subsubsection{Outline of the Thesis}

The remaining part of this document is arranged as follows. This report contains six chapters. Chapter 2 covers the background and related work on V2V communication and 
some of its application. The RoAdNet protocol is explained in Chapter 3. Chapter 4, gives an overview of the tools used to set up the experiment. The 5th chapter summarizes the results and discussions. Chapter 6 has the conclusion. 


\section{Chapter 2}

\section{Background work and existing literature}

In this chapter, we discuss existing literature for data dissemination and aggregation using structured and structureless protocols over wireless networks. We also take a look at recent advancements in $\mathrm{V} 2 \mathrm{~V}$ technology in terms of protocols, use cases and applications.

Improvement in hardware technology has enabled the availability of low power wireless devices, which have high computing power, more memory, and enhanced wireless communication systems. This has resulted in an extensive study of wireless protocols over the last decade. The research aims at designing protocols that can utilize the computing power of wireless nodes to have high data throughput and at the same time be more energy efficiency.

Wireless protocols used for data dissemination or aggregation are broadly categorized into two different types, (i) Structured and (ii) Structure-less. Structured protocols work by virtually re-arranging the nodes into structures like trees and graphs. They may choose to either maintain the tree or periodically refresh it. Also, the nodes can continuously send data or when they receive data requests. Structured protocols are shown to perform very well for static networks, i.e. networks were nodes are not mobile. Structureless approaches on the other have a distinct advantage over structured protocols when used in mobile networks. Structureless protocols rely on techniques such as flooding and gossiping as mobile nodes result in a rapidly changing network topology and maintaining a tree or a graph can have significant data overheads [3]. Next, we will discuss some structure-based approaches. 


\subsection{Structure-based Protocols}

As said earlier, structure-based approaches are used in static networks. Their applications include data aggregation, targeted data collection etc. Studies have shown that for information aggregation, the use of spanning trees is an efficient solution $[4,5,6,7]$. General working of such protocols depend on two types of messages. Firstly, the data packet, which carries the information that is being collected. Secondly, a control packet, which contains information to enable nodes to organize themselves into trees and graphs.

\subsubsection{Directed Diffusion}

Directed Diffusion [7] is a structured protocol which depends on a data request from the nodes to initiate data transfer. The requests are made in form of interests for named data. The node receiving the request finds the matching data and routes it towards the requesting node. Tasks are named using Attribute-value pairs. Data is transmitted only one hop at a time. Directed diffusion adopts some important concepts like gradients, datagrams, interests, and reinforcement. Reinforcement is the process of flooding a query from a node for the rest of the network. This helps to initialize the network gradients. The routing algorithm uses the gradients to find a path to the node that sent a request.

Nodes in the network maintain a cache where they store information about the query and their one-hop neighbor, along with a gradient or link effectiveness value. This also helps the node to drop duplicate data requests.

When a node needs information, it starts reinforcement with its first hop neighbors, who in turn reinforce their neighbors on the next level. Reinforcement stops when the desired node receives the data request message.

\subsubsection{Tiny AGgregation}

Tiny Aggregation Service for Ad-Hoc Network Service[8] (TAG) was proposed by Madden et. al. TAG is a declarative interface for data collection and aggregation. Its main focus is on achieving power efficiency by distributing and executing queries. It also uses a tree-like structure to rout data back to the base station. The data is aggregated on its way to the base 
station depending on the aggregation function. This helps reduce the number of messages in the network. TAG nodes advertise them self with a control message. The neighbors, on hearing them can decide if they want to choose the node as a parent node. A broken link can be caused when a node is removed from the network, if a node dies due to running out of battery power or if environmental conditions cause degradation of link quality. In such a case, TAG can detect a broken link if a node does not hear from its parent for a long time and start a repair process. How fast the link is repaired depends on the rate of transmission of the control signal.

Let us now take a deep dive into another commonly used protocol to, CTP (Collection Tree Protocol)[6] get an in-depth understanding of how such a protocol works.

\subsubsection{Collection Tree Protocol - CTP}

$\mathrm{CTP}[6]$ is a best effort datagram communication protocol. It was designed for low to moderate data traffic. Any node in the network can be designated as the base station (BS) or root. BS advertise themselves. Remaining nodes in the network try to form the best path to the base station. A network can have multiple base stations and data may be sent to any of the base stations. Depending on which base station the data is targeted to, the transmitting node may select an appropriate next hop neighbor. CTP maintains a tree once it is formed.

The routing gradient used is Expected Transmissions or ETX. Base stations normally have a Zero ETX. ETX for Intermediate nodes are real numbers. the ETX of a node is defined as:

\section{Node ETX $=E T X$ of parent $+E T X$ of its link to parent}

Nodes boot up with an infinite ETX value, except the root. Control packets are periodically transmitted by all nodes. Other nodes receive the control packet and update their ETX. The base ETX gradient increases by a factor of 10 on every hop. Link factor is used to fine tune the ETX. Re-transmissions are commonly used to account for dropped packets. A higher number of re-transmissions implies a lower link factor, which in turn implies a lower ETX. If a node has multiple neighbors, data is generally transmitted via the neighbor with 
higher ETX.

Packet drops and routing loops are a major concern with wireless protocols. ETX can be used to fix such undesirable behaviors. Fixing broken links need higher beaconing rate whereas a stable network can pull down on the beaconing rate. A trade-off on the beaconing strategy is achieved by using Adaptive Beaconing. A Beaconing strategy similar to Trickle[9] is used. It helps in faster network recovery while consuming low bandwidth.

Data is transmitted as datagrams. The routing and forwarding engine takes care of routing the data to the root. It randomly picks up a transmit time within a window for the next hop. An acknowledgment (ACK) is expected from the receiver for each transmitted packet, within a predefined time interval. In case, the transmitted packet or the ACK packet is lost the node re-transmits packets up to 32 times before it gives up and drops the packet. ACKs play an important role in suppressing duplicate packets. A duplicate packet can multiply exponentially in the subsequent hops. The nodes store received and transmitted packets in buffers to ensure hassle-free data flow. Cache size is chosen considering a tradeoff between network reliability and available memory. CTP has been widely used for data collection. Here at West Virginia University, CTP was used to implement a data collection mechanism from smart bricks with wireless sensors to estimate the health of a slagging gasifier.

\subsection{Beaconing}

Managing the beaconing rate in a wireless networks is critical to maximizing the throughput of the network. In a all-to-all network with $\mathrm{N}$ nodes the number of messages in the network increase at a rate of $N^{2}$. This can quickly consume the entire bandwidth resulting in collisions and dropped messages. Beaconing strategies like Trickle[9] help in optimizing the bandwidth usage in static networks. Trickle is a adaptive beaconing strategy where nodes change the beacon rate depending on the state of the network. Trickle slows down the beaconing rate when network links are stable. On detection of a broken link the beaconing rate resets to maximum and slowly reduces the rate as the network becomes stable.

Abramson, proposed ALOHA[10], a broadcasting strategy for radio communication which 
was as simple as transmitting a data packet when it was ready to be transmitted. It was not a very effective method of transmission as data was frequently as ALOHA does not check to see if the channel is free. Slotted ALOHA[11], is an improved version of ALOHA, where messages are transmitted in discrete time slots, by randomly choosing a time slot. This significantly improves the throughput of the network.

\subsection{Structureless Protocols}

Structureless protocols are relatively less researched. They are very useful in scenarios where nodes are mobile. The fact that they do not have to maintain the tree significantly reduces message overheads. Flooding and Gossiping[12] based approaches have been used to design such protocols. Random walk based ideas are seen as a possible alternative to implement such protocols

\subsubsection{Flooding and Gossiping}

Flooding data can be expensive in terms of the number of messages and the cost can be of an order of $O\left(N^{2}\right)$. Multiple local gossips can be used to get local consensus and this can be repeated till convergence[13]. This has been shown to have high communication costs and long convergence time. Spatial gossip techniques such as [14] and [15] can be used to choose neighbors randomly with a certain probability. All nodes learn about the state of the network after $O\left(\log ^{1+\epsilon} N\right)$ repetitions, which includes $O(N \cdot \operatorname{polylog}(N))$ messages.

\subsubsection{Random Walks}

Random walks are a method of exploring a set of nodes without following a pre-defined path. The nodes can generate tokens and pass it to all or some neighbors chosen at random. Over a period of time, the token will eventually cover the entire network and contain the aggregate network information. Multiple random walks can be used to cover a single network. Studies in $[16,17,18]$ have shown that by using $k$ independent random walks the cover time can be reduced by a factor of $k$. 
Work has been done in West Virginia University's (WVU) Wireless Networks Lab(WNL) on push-pull based random walk protocols aimed towards efficiency in data aggregation in mobile networks[19]. This protocol starts by generating one or more tokens at randomly selected nodes. The nodes forward the token to a randomly selected neighbor. When a node sees a token for the first time it adds its own data to the token. On subsequent arrival of the same token, it is just passed ahead. The fact that a node can be visited multiple times results in a typically high cover time (time to visit all nodes). Partially guided random walks help improve cover time in a network. Here the nodes pass the token to one of its randomly chosen un-visited nodes. This biasing has shown to improve the cove time to $O(N . \log (N))$. In either case, a slow down is noticed after a significant number of nodes have been visited, resulting in a long tail. This is caused by the token getting trapped in regions that have already been explored.

\subsection{Vehicular Communication and Applications}

In recent years, there has been an increased interest in the development and evaluation of Vehicle to Vehicle(V2V) and Vehicle to Infrastructure (V2I) protocols. With the increase in automation and safety concerns, $\mathrm{V} 2 \mathrm{~V}$ is expected to play a major role in making driving more reliable and safer. The United States Department of Transportation (USDOT) estimates that more than 17,000 fatalities in the first half of 2018[20], which included over 1.6 Million crashes. It also estimates that over 0.6 Million of these could be averted by use of V2V and V2I technology.

V2V scenarios can be a harsh environment for radio communication. Considering high mobility, an uncontrolled and dynamic environment and multipath effects, a modified version of Wireless LAN was proposed by IEEE called the IEEE 802.11p, more commonly known as DSRC(Dedicated Short Range Communication), for V2X applications. The US Federal Communication Commission(FCC) allocated a dedicated $75 \mathrm{MHz}$ bandwidth in the 5.850 to $5.725 \mathrm{Ghz}$ band.

In this section, we will discuss existing research on various aspects of V2X communication. These include communication protocols that have been proposed based on DSRC 
and cellular networks, Advanced Driver Assistance Systems (ADAS), adaptive cruise control(ACC) mechanism and impact of vehicular communication on navigation, emissions, and environment.

\subsubsection{DSRC Based Protocols for Vehicular Communication}

As discussed earlier, VANETS are a special category of MANETS with constraints on mobility and well-defined movement patterns. Hence numerous VANET protocols have been proposed based on classical MANET protocols like Ad-Hoc On-Demand Distance Vector Routing(AODV)[21, 22] and Dynamic Source Routing(DSR)[23]. The efficiency of such protocols for VANETS can be further improved by including position or localization information from GPS or Map-based systems.

Tee and Lee proposed Junction-based Adaptive Reactive Routing (JARR) for VANET in city environments [24]. JARR is designed for forwarding packets between junctions. Nodes announce their position and speed. Depending on an estimated density of nodes, JARR selects a path to the next junction. Scalability of JARR is limited as the distance between the junctions have to be calculated and stored prior to using the algorithm. Greedy Perimeter Stateless Routing(GPSR)[25] used control beacons to intimate each node about the location of all other nodes in the network. When a node $x$ received a packet that is destined for $y$, it forwards it to a node $z$ whose distance to $y$ is less than distance between $x$ and $y$. GPSR can dynamically switch to perimeter mode if it does not find any qualified neighbor. The fact that all nodes need to know about their neighborhood makes it vulnerable when mobility is very high.

Zhao and Cao propose various Vehicle-Assisted Data Delivery (VADD) [26] protocols. It is based on the idea of carry and forward. VADD adjusts well in sparse density scenarios. If a wireless route is not available between source and destination, the packet can be carried forward for some time by a vehicle and then dispersed into the network when density conditions are ideal. The possibility of effectively selecting sections of road intersections to maximize the chance of a packet being delivered to an internet gateway can be seen in Saleet et al.'s work. [27]. 
Beaconing strategies play a crucial role in the success of communication protocols. In high-density roads, it is essential to have a robust beaconing policy to avoid packet drops and low throughout [28]. Allouche and Segal propose a cluster-based beaconing for VANETS [29]. It divides the communication into intra-cluster and inter-cluster protocols. While inter-cluster is very similar to any other wireless data dissemination protocol, the intracluster protocol is a one-hop aggregation protocol and has a vehicle proximity map. This significantly reduces the number of data packets, resulting in high throughput.

\subsubsection{Cellular Based Vehicular Communication}

Cellular-based V2X communication or C-V2X relies on the availability of cellular networks to share information within vehicles. Although direct communication between vehicles is still an active field of research, cellular-based techniques have long been used for data aggregation and information procession a centralized server. Applications such as Google maps and Waze rely on crowd-sourcing data from mobile smartphone users to predict traffic flows[30].

A major challenge with $\mathrm{C}-\mathrm{V} 2 \mathrm{X}$ is that they are dependent on cellular infrastructure. Their performance will degrade in areas of low or no coverage. Also, setting up cellular infrastructure is not cheap. With multiple service providers, there is a need for a common framework.

\subsubsection{Applications of V2V Communication}

The automotive industry is rapidly moving towards automation aided by advancements in Advanced Driver Assistance Systems (ADAS) features. Five distinct levels of automation, from Level 0 (no automation) to Level 4 (full automation) have been specified by the National Highway Traffic Safety Administration (NHTSA)[31]. ADAS features currently available to drivers include Adaptive Cruise Control (ACC), blind-spot warning, lane departure warning, pre-crash warning, and automatic braking[32]. These systems rely heavily on a sensor suite to map the surrounding and take control decisions. The sensors used are the camera, radar, and LIDAR. The limitation of these sensors is that they can only map the surrounding in 
the line of sight.

Let us consider collision avoidance systems. Research has shown how the availability of information and network latency affect collision avoidance systems[33]. Also, Liu and Watta in their research about pre-crash detection and warning using V2V communication [34] stresses the importance of the context of the driving environment. They propose an algorithm to compute the relative position of all vehicles with respect to the host vehicle. Geo distance formulae were used for distance estimation from GPS coordinates. The crash detection system worked particularly well considering minimal information used as inputs to the algorithm.

Platooning is seen as an eco-driving strategy, especially for heavy-duty vehicles. Cooperative Adaptive Cruise Control (CACC) along with V2V communication can be used to enable platooning. Collaborative driving has to follow a rigorously planned architecture. Different levels of abstractions like vehicle control, traffic control and management will be needed as cited in [35]. This was also demonstrated with a five-vehicle platooning[36]. The architecture in [37] has a hierarchical architecture, with guidance, management, and traffic control layers. Studies in [38] and [39] have shown that platooning can have a significant impact on efficiency and emissions. Up to $14 \%$ fuel can be saved and $\mathrm{CO}_{2}$ emission can be reduced by up to $2.1 \%$ when $40 \%$ of heavy trucks have the platooning capability.

String stability is a key factor for vehicle platooning[40]. In a string stable platoon, disturbances must be suppressed. Although some disturbances will propagate through the platoon, they must not grow unbounded. Besselink and Johansson [41] recommend a delay based spacing policy for platoons subject to disturbances. Instability can arise from unfavorable vehicle dynamics or packet loss in connected vehicles.

Availability of information from long range (up to 10kms) can help hybrid electrics make intelligent control decisions. Internal combustion engines are less efficient than electric motors[42]. Hadi et al. have shown that real-time powertrain control strategies can be improved to increase performance in terms of fuel efficiency, number of engine transients, and charge sustainability of battery by using predicted driving condition[43]. Alternatively, the information to be predicted can be made available directly via V2V. [44] work on using traffic-light-signal-to-vehicle(TLS2V) information to recommend driving speeds have shown 
to help reduce fuel consumption and emissions.

\subsection{Adaptive Cruise Control \& Car Following Models}

Cruise control is an essential sub-system of automation. It refers to the ability of a vehicle to maintain a constant speed without tipping into the accelerator pedal. An advanced version of cruise control, known as Adaptive Cruise Control (ACC), tries to maintain a constant speed, in the absence of a lead vehicle or when the lead vehicle is far ahead. ACC switches to gap control mode, depending on the relative velocity and distance to the lead vehicle.

Various control algorithms have been proposed for ACC systems. Model predictive control algorithms proposed in [45] and [46], predict the vehicle acceleration and deceleration and uses the predictions to generate control commands. Fuzzy logic based control ideas have also been used to improve ride comfort by minimizing jerk[47]. A more advanced control mechanism called the Co-operative Adaptive Cruise Control(CACC) has been studied in the recent past. It is an extension to the ACC and makes it more efficient by using information about the preceding vehicle which can be obtained via V2V. CACC can help achieve time gaps of 0.6-1.1 seconds as compared to 1.1-2.2 seconds for ACC [48] and also has the potential to improve fuel use up to $25 \%$ and reducing delays by up to $91 \%$ [49].

A similar concept in traffic flow model is called Car Following Models (CFM). CFMs are an essential part of microscopic traffic modeling. They define how individual units, cars, in this case, behave in traffic[50] [51]. The models may include behavioral models for acceleration, cruising, slow-moving and stationary situations. CFMs take sensor information about the leading vehicle and use model-based algorithms to predict the following speed, acceleration and de-acceleration of the vehicle. Various car following models have been proposed since the early 1970s.

Assuming that only change in velocity is performed, the simplest dynamics can be described by

$$
\frac{d v_{i}(t)}{d t}=\frac{v_{i+1}(t)-v_{i}(t)}{\tau}
$$

To avoid collision it is obvious that the speed of vehicle $i$ should be at most equal to the speed of the leading vehicle $i+1$. This was first proposed by pipes [52]. Gipps first considered 
modeling the braking distance to CFMs [53]. The braking capability of the vehicle was underestimated so that the model can describe the overreaction of drivers is when suddenly faced with hazardous conditions.

Krauss proposed that for traffic flow to be modeled, two factors should be considered. Firstly, the free motion of the vehicle and secondly, its motion when it interacts with other vehicle [54]. He also stressed on the importance of discrete models, which would be easier to implement in a simulation environment. In discrete simulations, the next speed of a vehicle can be determined by

$$
v_{\text {next }}=\min \left[v+a_{\max } \times \Delta t, v_{\max }\right]
$$

Where $\mathrm{v}$ is the current vehicle speed and $\Delta t$ is a simulation time step. $a_{\max }$ is the maximum acceleration of the vehicle until it reaches the velocity $c_{\max }$. Adding the lane speed limit factor gives us

$$
v_{\text {next }}=\min \left[v_{\text {next }}, v_{\max }^{A}\right]
$$

The discrete event simulator, SUMO, described in the next section uses a similar approach for calculating the vehicle's speed and position[55]. 


\section{Chapter 3}

\section{RoAdNet Protocol Design}

In this chapter, we will dissect RoAdNet to understand its working and highlight various salient features. RoAdNet is designed to have the following features.

1. Broadcast single hop information at high frequency, with high precision

2. Send Multi-hop information, up to $1500 \mathrm{~m}$, with a distance based reduction in transmission frequency

3. Provide aggregate information beyond $1500 \mathrm{~m}$ and up to $10000 \mathrm{~m}$ with low latency

4. Seamlessly transition between the three modes of working

For ease of understanding, we will divide the protocol into four main modules as seen in Fig. 3.1. First, the Transmit Engine(TE), which is responsible for creating and transmitting source packets at high frequency. Second, the Receive Engine(RE) which handles packet receptions. Third, the Forward Engine(FE), which takes care of forwarding multi-hop packet. Fourth, the Aggregate and Adapt Engine (AAE), is responsible for sending aggregate information over long distances. RoAdNet runs all the engines as independent and parallel threads. In the rest of this section, each of these engines is described in detail.

\subsection{Transmit Engine}

The Transmit Engine (TE) is the simplest module of the protocol. It is invoked at a pre-defined high frequency of $5 \mathrm{~Hz}$ or $10 \mathrm{~Hz}$. The tx_beacon_event_timer is responsible is used 


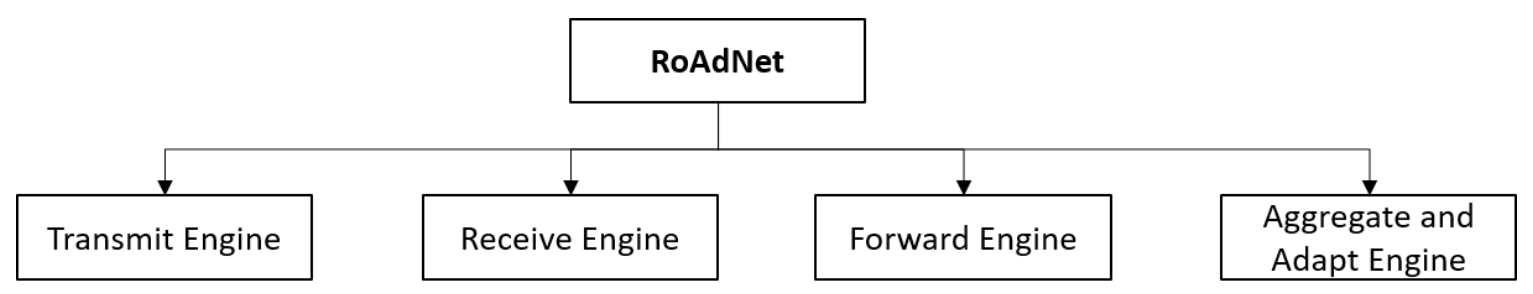

Figure 3.1: Modules in RoAdNet

to define the frequency of the transmit engine. The main task of the TE is to create a data packet, populate it with relevant information and schedule it for transmission. Transmissions are scheduled with a certain randomness to avoid packet collisions. The data packet contains essential information that can be used by other vehicles for safety and navigation purpose. Table 3.1 lists the content of the data packet.

The cell_speed, cell_density, cell_id contain aggregate information about a geographic region or a particular street.

$$
\begin{aligned}
& \text { transmitEngine }(\text { data_packet }) \\
& \text { packet }=\operatorname{createDataPacket}() \\
& \text { delay_ms }=\text { random }\left(0, \zeta_{t x}\right) \\
& \text { scheduleForTransmission(packet, delay_ms) }
\end{aligned}
$$

\section{Algorithm 1: Transmit Engine}

Packet collision is a major concern in shared transmission medium. Collisions occur when two nodes transmit at the same time. In order to minimize the chances of collision the TE uses Randomized Transmission Schedules (RTxS). RoAdNet defines a transmission spread $\left(\zeta_{t x}\right)$, which typically ranges between 100-200ms depending on the desired base transmission frequency. When a packet is ready to be transmitted, it is scheduled at a time-stamp randomly picked within the next $\left(\zeta_{t x}\right)$ spread. Every scheduled packet is put in a transmission queue. Once the timer for each packet expires, it is sent out in the network. 


\begin{tabular}{|c|c|l|}
\hline Content & Data Type & Description \\
\hline \hline sender & string & Name of the sender \\
\hline recipient & string & Name of the receiver \\
\hline pos & TraCICoord & (x,y,z) position of the sender \\
\hline speed & double & Speed of the sender \\
\hline accel & double & Acceleration of the sender \\
\hline maxDecel & double & Maximum deceleration of the sender \\
\hline lane & String & Current lane of the sender \\
\hline angle & double & Current angle of the vehicle \\
\hline brakes & unsigned int & Brake status of the vehicle \\
\hline message_id & string & $\begin{array}{l}\text { A message ID unique to every mes- } \\
\text { sage in the network. Consists of VE- } \\
\text { HICLEID_MESSAGECOUNTER }\end{array}$ \\
\hline hop & double & $\begin{array}{l}\text { Number of hops the message has trav- } \\
\text { elled so far }\end{array}$ \\
\hline cell_speed & double & Average speed of all vehicles in cell \\
\hline cell_density & double & Number of vehicles in cell \\
\hline cell_id & String & $\begin{array}{l}\text { Type of cell. e.g. Street, Geographical } \\
\text { region, etc. }\end{array}$ \\
\hline
\end{tabular}

Table 3.1: Contents of data packet

\subsection{Receive Engine}

The Receive Engine(RE) receives and processes all the packet it receives. The pseudoalgorithm for the RE is given below. 


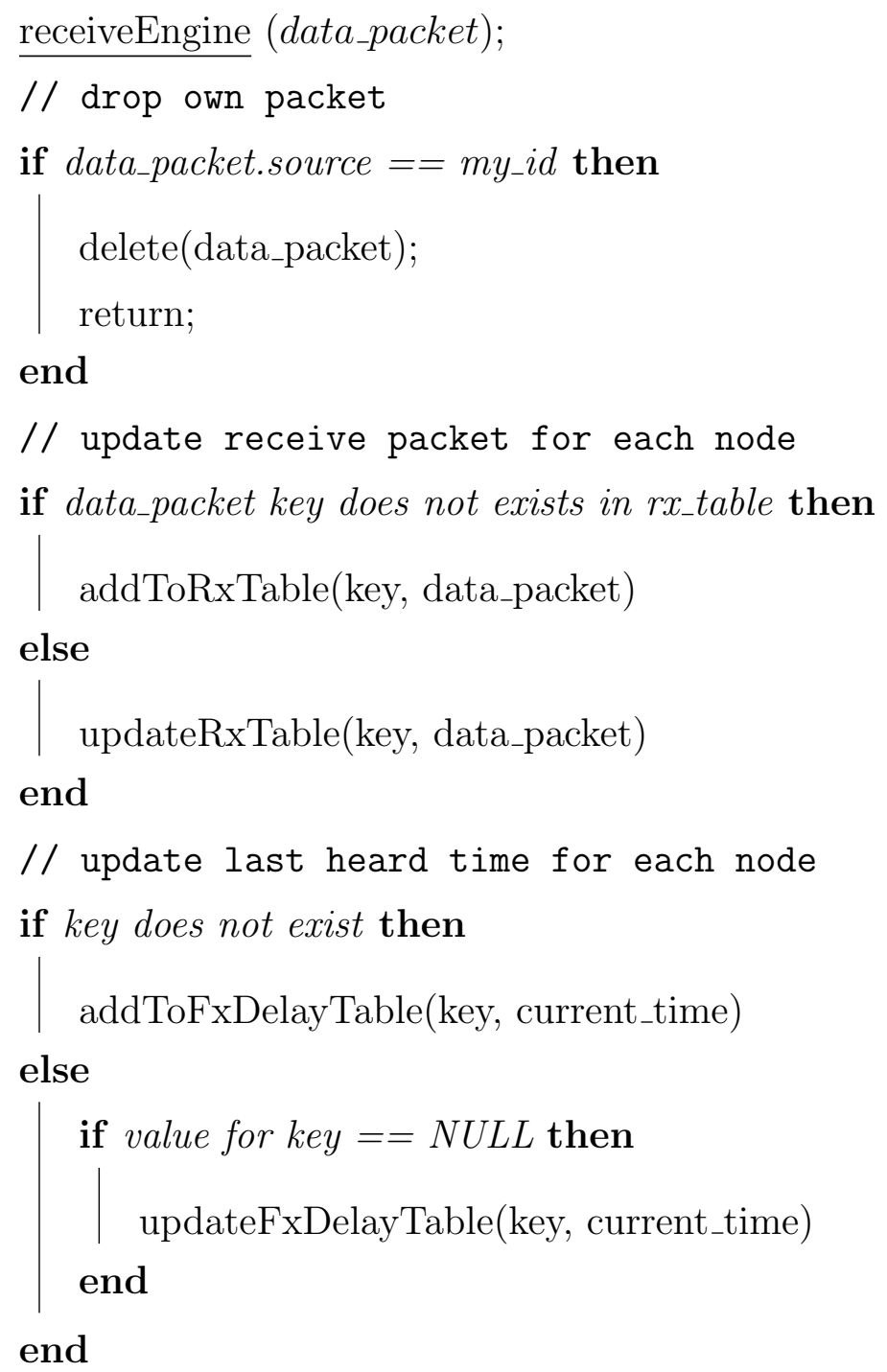

\section{Algorithm 2: Receive Engine}

The RE is evoked every time a vehicle receives a data packet. The packet is dropped/ignored if it originated from the same vehicle. If the packet is from a different vehicle it is processed further. If the packet is received from a vehicle for the first time, an entry is created in the rx_table. At any point in time, the rx_table contains the latest information about all the nodes. The time when the last information was heard from a node is stored in the fx_delay_table. This table is later used by the Forward Engine to induce distance based latency in the messages. An entry is created in this table when information about a vehicle is received for the first time. For subsequent messages from the same vehicle, the last heard time is updated every time. 


\subsection{Forward Engine}

The Forward Engine (FE) is the most critical module of RoAdNet. It is responsible for forwarding messages beyond the single hop communication range. The frequency at which FE is invoked is a trade-off between network usage and latency.

\subsubsection{Traditional Forwarding Engine}

A traditional FE, when invoked, forwards all the packets in the database one after another in a single shot. This works very well when the base transmission frequency is low, which means, packets come in at large intervals and hence there is less number of packets to forward. After a certain threshold, we start losing messages due to the collision of packets. Hence the throughput of the network goes down.

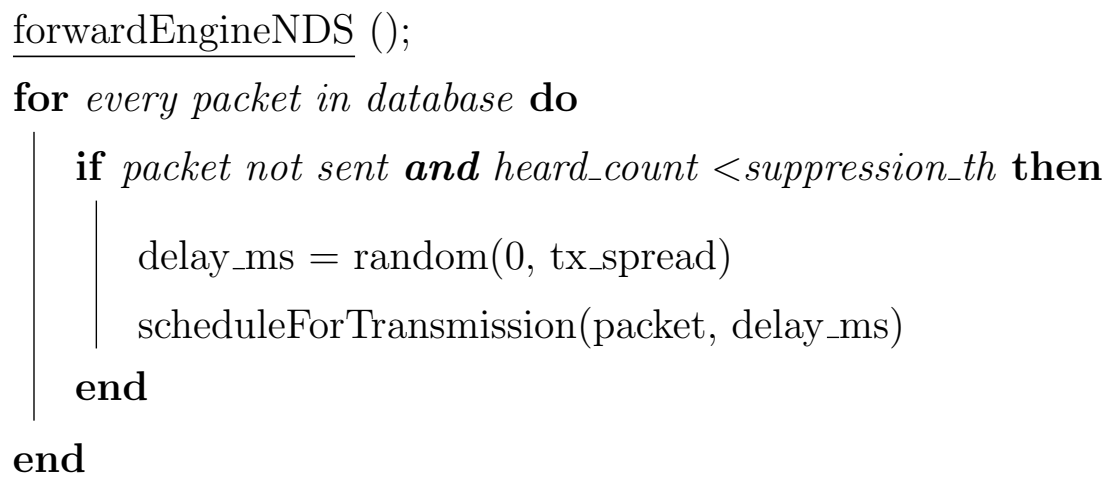

Algorithm 3: Forward Engine - Non Distance Sensitive

The FE iterates through the receive queue $\left(Q_{j}\right)$, where all received packets are stored and wait for forwarding. It forwards the packets if the time lapsed since last transmission about a node is greater than the Distance Sensitive Delay $\left(\delta_{p}\right)$ for the packet. $\delta_{p}$ is proportional to the distance of originating node form the current node. The staggering rate can be varied by changing the sensitivity factor $(\alpha)$.

$$
\delta_{p}=\frac{d_{i j}}{\alpha}
$$

where,

$$
d_{i j}=\text { distance between node } i \text { and } j
$$




$$
\alpha=\text { sensitivity factor }
$$

To utilize the available bandwidth more efficiently, RoAdnet draws inspiration from distance sensitive forwarding mechanisms.

\subsubsection{Distance Sensitive Forward Engine}

The distance sensitive forward engine forwards data packets at a rate proportional to its distance from the point of origin. To do so, for every packet to be transmitted the time_elapsed_for_node is calculated, which is the time since the last packet was forwarded from the node. Next, its distance to origin is calculated as dist_to_origin. This is used to find the distance sensitivity factor $(\alpha)$ which is a time penalty in forwarding the packet. The information about the node is only transmitted if time_elapsed_for_node is greater than $\alpha$. The sequence in which the packets will be sent out can be seen in Fig.3.4. It can be seen that when Vehicle 3's forwarding function is triggered, it first sends out the messages from vehicle 4 and 2 only, as they are in they are within the first bucket/bin for vehicle 3 . On subsequent trigger of the forwarding function the messages from vehicle 1 and 5 are transmitted along with messages from vehicle 2 and 4 .

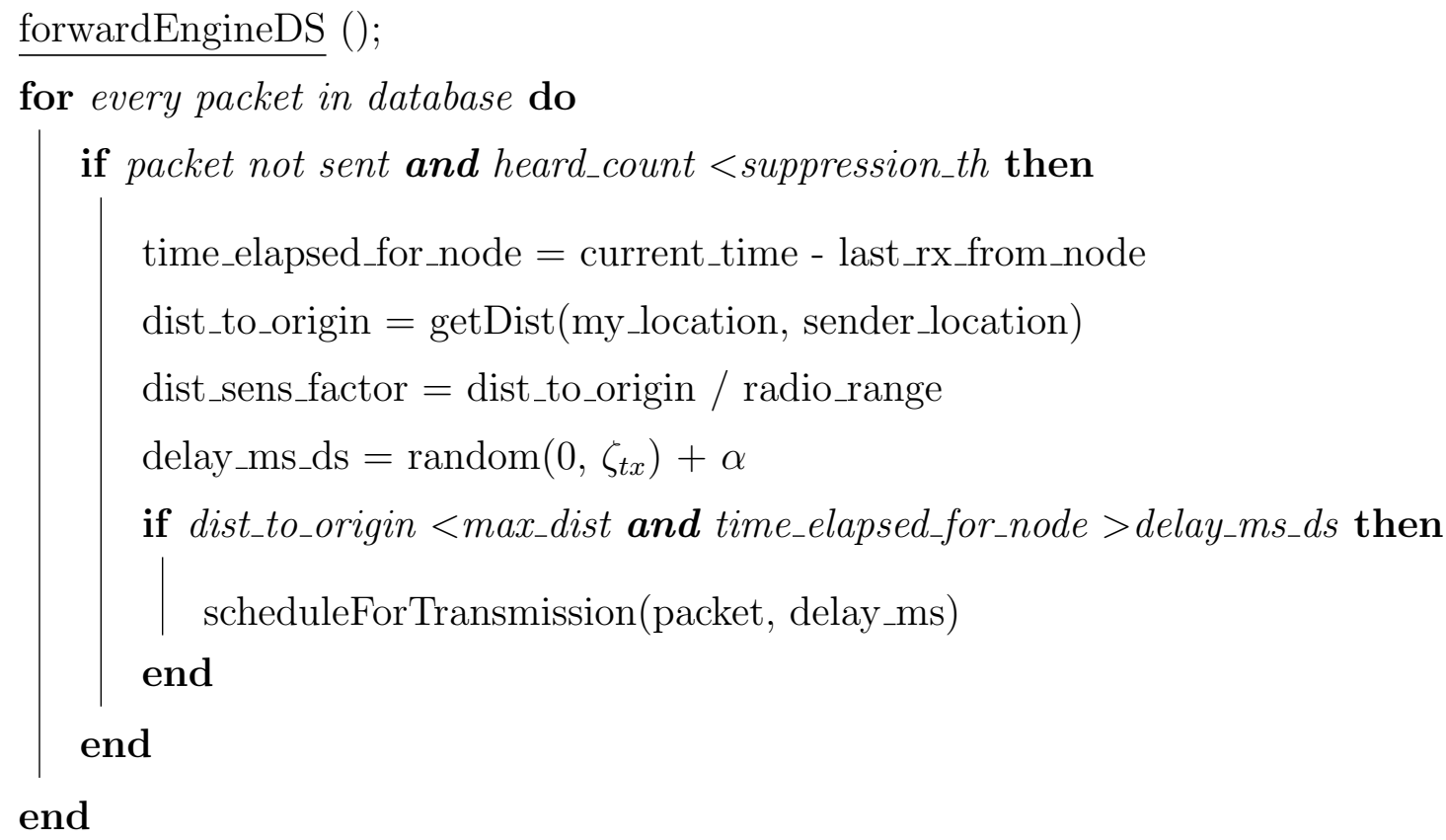

Algorithm 4: Forward Engine - Distance Sensitive 
To better understand distance sensitive forwarding let us consider five nodes with uniform radio ranges, as shown in Fig 3.2.

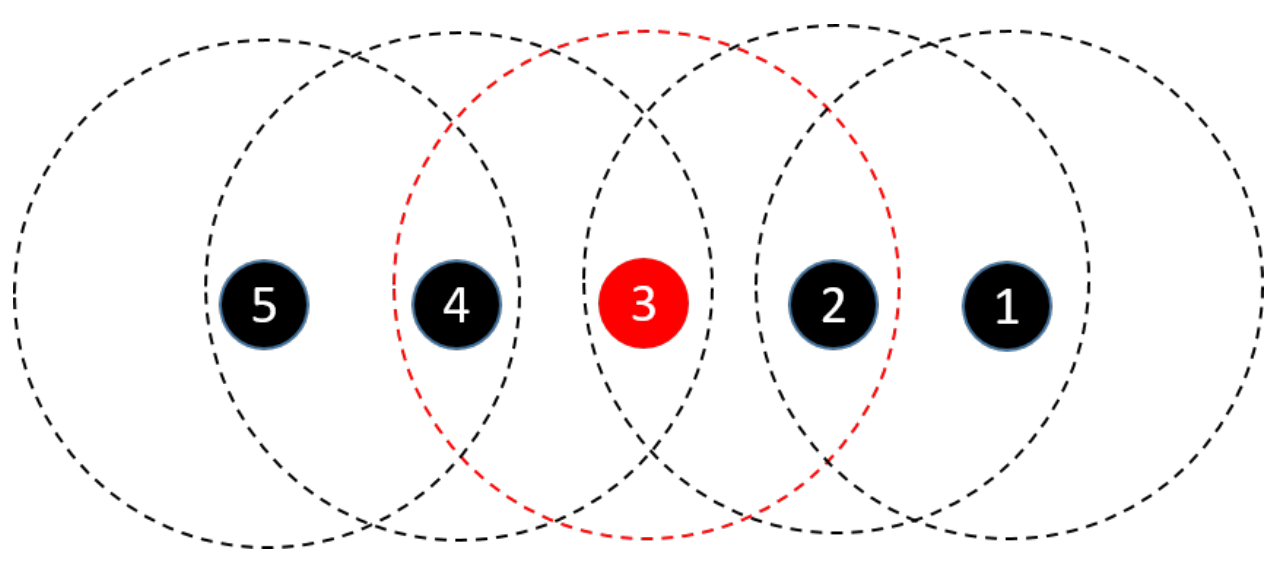

Figure 3.2: Position and radio range of vehicles

Let us consider node 3 as our node of interest. When the forward engine (FE) is triggered on the node it schedules forwarded messages from node 1,2,4 and 5. It can be seen in Fig. 3.3, for a Non Distance sensitive forwarding scheme, Node 3 schedules messages from 1,2,4 and 5 on a single FE trigger.

The Distance Sensitive mechanism used by RoAdNet, schedules the messages depending on the distance of the origin node from Node 3. It can be seen in Fig. 3.4 that node 3 schedules messages from node 4 and 2 on the first trigger as there are only 1 hop away from node 3 . On the successive trigger it sends out information about nodes 1 and 5 as they are farther away.

\subsection{Aggregate and Adapt Engine}

The Aggregate and Adapt Engine (AAE) is a low frequency periodic process which compares the state of each cell to its previous states and flags an event if a considerable change in cell state has occurred. In case of an event, an aggregation event packet $\left(E_{k}\right)$ is transmitted. AE uses a suppression technique similar to FE. Aggregation packets are 
$t_{0}$

\begin{tabular}{|l|l|l|l|}
\hline 5 & 4 & 2 & 1 \\
\hline
\end{tabular}

On Next

Fx

Fx

Fx

Fx

FE Call at $\mathrm{t}_{1} \quad(\mathrm{t} 1+$ rand $) \quad(\mathrm{t} 1+$ rand $) \quad(\mathrm{t} 1+$ rand $) \quad(\mathrm{t} 1+$ rand $)$

Figure 3.3: Transmission sequence of packets in traditional forwarding

\section{3}

$t_{0}$

\begin{tabular}{|l|l|l|l|}
\hline 5 & 4 & 2 & 1 \\
\hline
\end{tabular}

On Next

Fx

FX

FE Call at $t_{1}$

(t1 + rand $) \quad(\mathrm{t} 1+$ rand $)$

$\begin{array}{rc}\text { On Next } & \text { Fx } \\ \text { FE Call at } t_{2} & \text { (t2 }+ \text { rand })\end{array}$

Fx
$(\mathrm{t} 2+$ rand $)$

Figure 3.4: Transmission sequence of packets in Distance Sensitive Forwarding

transmitted up to 10,000 meters. The AE transmits cell state packets at low frequency. Occasionally, it transmits event packets at high frequency in short bursts.

The aggregation of information starts in the Receive Engine (RE). The RE on receiving a packet, scans through the contents and decodes all the information about the transmitting node. It uses the location information to map the vehicle to a cell number $k$. RoAdNet maintains the status of all cells in the geographical region and updates the cell state on reception of every packet. Cell state includes average vehicle speed and average travel time in a cell. A change in cell state over a certain period of time can trigger an event in the network.

Events in the network can be triggered in two different ways. (1) A vehicle can trigger its own event, when it is involved in an accident or a hazardous situation that poses potential 
danger to other vehicles on the street. (2) The AAE can also generate an event when it senses a considerable change in cell state. A change in cell state can be a change in the average speed of vehicles in the cell, beyond a certain threshold. The threshold would define how frequently events are triggered.

In a stable state, the AAE transmits cell state information at a very low frequency of $1 / 10$ Hz. On sensing an event (unstable cell), the transmission frequency switches to transmitting event packets at a higher frequency of $10 \mathrm{~Hz}$. The event packet is an extension of the cell state packet with a event byte, which contains information about the type of event. As the cell regains its stability the transmission frequency goes back to low. This is important as most of the cells are stable over long periods of time and occasionally trigger event. The cell state message being low frequency should not affect the staleness of base transmissions. The event message, is high frequency, but it is a burst only for a very shot time. This also has minimal affect on the base transmissions.

The contents of the Aggregate Cell State packet is shown in Table 3.2

\begin{tabular}{|c|c|l|}
\hline Content & Data Type & Description \\
\hline \hline cell_id & string & Unique cell identifier \\
\hline avg_speed & float & Average vehicle speed in cell \\
\hline avg_travel_time & float & Average travel time in cell \\
\hline event & Bool & Event Flag \\
\hline ev_type & chat & Type of event \\
\hline
\end{tabular}

Table 3.2: Contents of Aggrecation/Cell State packet

\subsection{Division of Cells for Aggregation}

The shape and size of the cells can be defined depending on the geographical region and density of the road network. For simplicity of RoAdNet evaluations the cells are defined as rectangular regions of size $0.25 \mathrm{~km}^{2}$ (squares of size $500 \mathrm{~m}$ each) as shown in Fig 3.5 . Information about all the vehicles in a cell is averaged to generate aggregate information.

The RoAdNet Protocol is independent of the cell definition and can adapt to different 
types of cells. Cells may be defined as a geographical region, a single street or a group of streets, depending on the application.

\subsection{Handling Packet Collision and Network Through- put}

Packet collisions are a common issue in shared medium. When any two nodes attempt to transmit a packet in the network at the same time, a collision occurs. RoAdNet relies on multiple techniques to minimize collisions and better utilize the available bandwidth.(1) Randomized Transmission Schedules (RTxS) (2) Multiple Packet Suppression (MPS) and (3) Distance Sensitive Forwarding (DSF).

\subsubsection{Randomized Transmission Schedules}

The TE and FE use randomized transmission schedules to transmit packets. RoAdNet defines a transmission spread $\left(\zeta_{t x}\right)$, which typically ranges between $100-200 \mathrm{~ms}$ depending on the desired base transmission frequency. When a packet is ready to be transmitted, it is scheduled at time-stamp randomly picked within the next $\left(\zeta_{t x}\right)$ spread. Every scheduled packet is put in a transmission queue. Implementing RTxS also allows the forward engine enable MPS, which is described below.

\subsubsection{Multiple Packet Suppression}

The FE and AE use Multiple Packet Suppression (MPS) to minimize the number of packets being transmitted resulting in lesser collisions and better bandwidth utilization. Lets say, vehicle $j$ has scheduled a forwarding packet $P$ from vehicle $i$ at time $t$. Vehicle $j$ actively listens to the network and keeps a count of how many times other vehicles within its communication range have transmitted the information about vehicle $i$. If the count hits a threshold before time $t$, the FE drops the packet $P$ from the transmission queue. 


\subsubsection{Distance Sensitive Forwarding}

The FE also used DSF to minimize the number of transmissions in the network. DSF is achieved by staggering packets at a rate proportional to the Distance Sensitive Delay as described in an earlier section of this report.

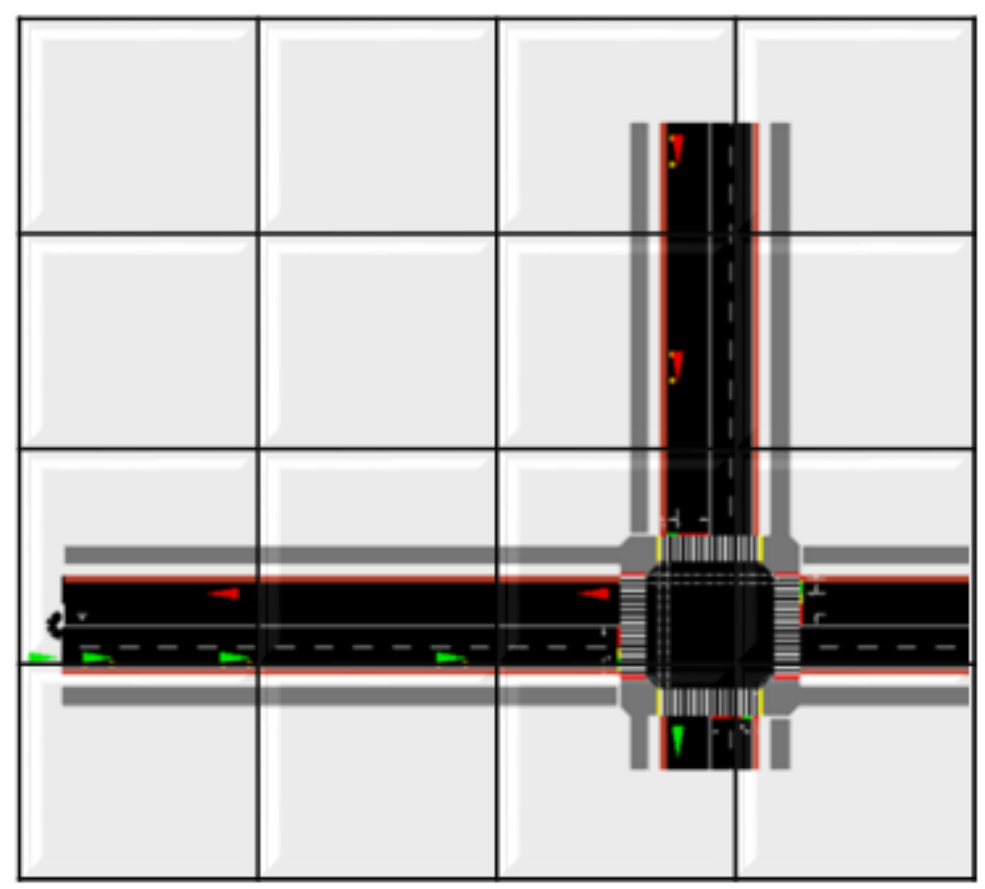

Figure 3.5: Cell design for RoAdNet Evaluation

\subsection{RoAdNet Algorithm}

Algorithm 5 shows the consolidated algorithm for RoAdNet. 


\section{Variables:}

$j$ : Current Node

$i$ : Transmitting Node

$k:$ Cell Id

$P_{i}$ : Data packet from node $i$

$E_{k}$ : Aggregate Event packet about cell $k$

$\tau_{i}$ : Time when packet form node $i$ was received

$\tau_{l h}$ : Time since packet was last heard from node i

$\delta_{P}$ : Distance sensitive delay for packet $P$

$Q_{j}$ : Received messages queue at node $j$

$\gamma_{k}$ : Average vehicle speed in cell $k$

transmitEngine ():

$\operatorname{send}\left(P_{i}\right)$

receiveEngine (data_packet):

if $P_{i}$.source $=j$ then

I return;

else

$Q_{j}$.update();

$\tau_{i}$.update();

$\gamma_{k}$.update();

end

forwardEngineDS ():

for $\forall P \in Q_{j}$ :

if $\tau_{l h}>\delta_{P} A N D \mathrm{n} \leq \lambda$ then

$\operatorname{send}\left(P_{i}\right)$;

end

aggregateEngine ():

if $\gamma_{k}$.changed () then

$\operatorname{send}\left(E_{k}\right)$;

end

Algorithm 5: RoAdNet Algorithm 


\section{Chapter 4}

\section{Experimental Setup in VENTOS}

The chapter gives an overview of the tools and software used to implement and study the RoAdNet protocol. As RoAdNet is a protocol targeted at VANETs, it was essential to choose a platform that supports testing of network protocols with mobility that mimic movement of vehicle in the real world. Such a platform should be able to :

1. Simulate road traffic patterns

2. Mimic highways, urban roadways and intersections

3. Interact with real world maps, like Google maps or OpenStreet maps and represent them in a way so that virtual traffic patterns can be simulated.

4. Control vehicle dynamics like speed, acceleration, time gap etc.

5. Implement a V2V wireless data dissemination protocol.

The simulator used in this research is VEhicular NETwork Open Simulator or VENTOS[1]. It is an open-source VANET simulator in $\mathrm{C}++$ which is used for studying traffic flows, V2V and V2X interaction using DSRC based wireless communication. VENTOS harnesses the power of two popular simulators. It integrates Objective Modular Netowrk Testbed in $\mathrm{C}++$ $(\mathrm{OMNeT}++)$, which is a $\mathrm{C}++$ based network simulator with Simulation of Urban Mobility (SUMO), which is an open-source, microscopic, continuous-space, discrete-time road traffic simulator. It harnesses $\mathrm{OMNeT}++$ 's power to simulate network protocols and use vehicle mobility models in SUMO to replicate node mobility in OMNeT++. 


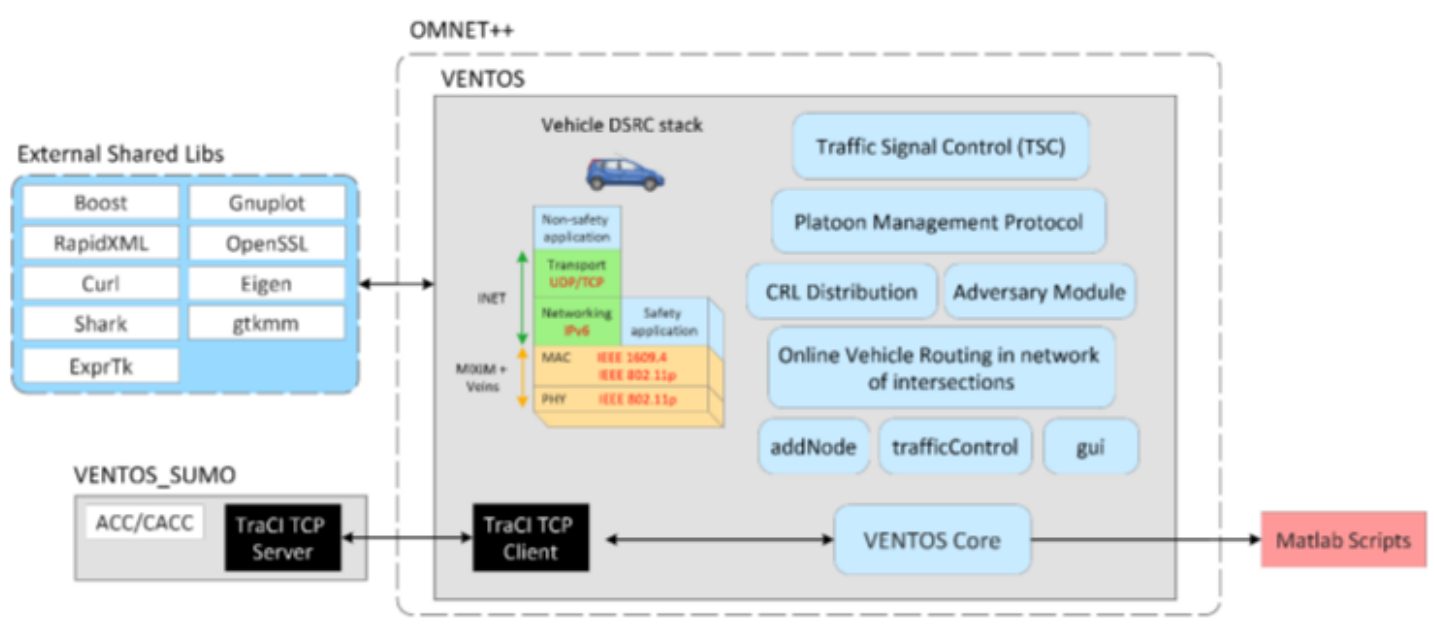

Figure 4.1: VENTOS Architecture[1]

Figure 4.1, shows the architecture of VENTOS. Functionalities of some of the major modules in the figure are:

1. VENTOS Core: It is the container for all internal modules and data logging. It is also responsible for initiating a TraCI (Traffic Control Interface) interface with SUMO.

2. TrafficControl: This module is responsible for controlling vehicle position and speed.

3. Traffic Signal Control: The module provides access to traffic signal control modules used in today's intersections. Some of the available algorithms are fix-time, longest queue first, oldest job first etc.

4. VENTOS SUMO: Has additional car following models for ACC and CACC in SUMO and is also responsible for TraCI communication with VENTOS core.

5. Online Vehicle Routing: Dynamic routing algorithms are implemented in this module.

6. Platoon Management Protocol: Platoon management protocols with platoon maneuvers such as merge, split, entry etc. are implemented in this module.

For studying V2X communication scenarios comprehensively, a dynamically changing microscopic traffic simulator is needed. The Traffic Control Interface or TraCI, allows external applications to retrieve information from SUMO's traffic simulator and use it in their 
simulations. Once VENTOS is started it creates a new process and then starts an instance of SUMO in TraCI mode. SUMO starts the simulation by loading the SUMO configuration file and starts a TCP server. It waits for the external application to connect ot it and request traffic data. When a node is inserted in SUMO it is replicated in OMNeT++. A timer is used by VENTOS to synchronize with SUMO. A TraCI command simulationStep is periodically called to advance SUMO simulation by one step. Hence, the nodes in OMNet ++ communicate via DSRC based radios, but their mobility is changed by SUMO through TraCI.

The first step to simulating a V2X scenario in VENTOS is to create road network and insert traffic flows in it. The following section shows different ways of doing this in SUMO.

\subsection{Creating a road network in SUMO}

In SUMO each street and vehicle can be modelled explicitly. It works with the help of two input files: Network file(.net.xml) and Traffic Demand File(.rou.xml). The network file defines roads and intersections. Every lane is unidirectional an can have a predefined position and speed limit. The traffic demand file contains descriptions for various vehicle types with different parameters like length, acceleration, maximum speed etc. SUMO represents lanes as edges and intersections as nodes. The route of a vehicle is specified in terms of nodes and edges.

\subsubsection{Creating network file}

There are multiple ways of generating a road network file in SUMO. Figure 4.2 shows the different methods and tools that can be used to generate a network file.

XML files can be manually created by defining the coordinated for lanes and intersections. For complex networks NETCONVERT and NETGEN can be used. NETGEN can generate abstract maps with random grids. NETCONVERT can be used to import Non-SUMO networks like OpenStreetMaps. Figure 4.3a and Figure 4.3b show two variants of 4 -way junctions designed using SUMO. A signalled junction has traffic signals to control traffic flow and a multimodal junction allows for multiple modes of transport. The movememt 


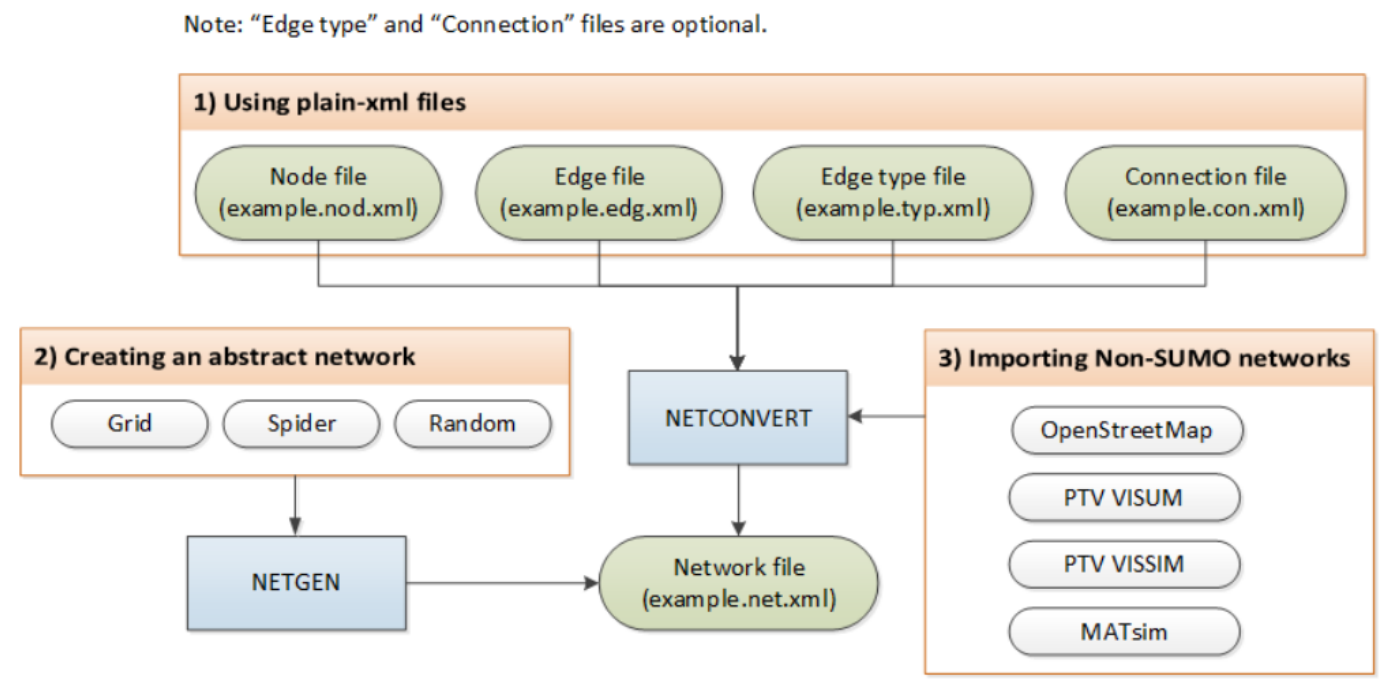

Figure 4.2: Methods of generating SUMO network file

of different modes e.g. vehicles, bicycle, pedestirans etc. are controlled by corresponding mobility models.

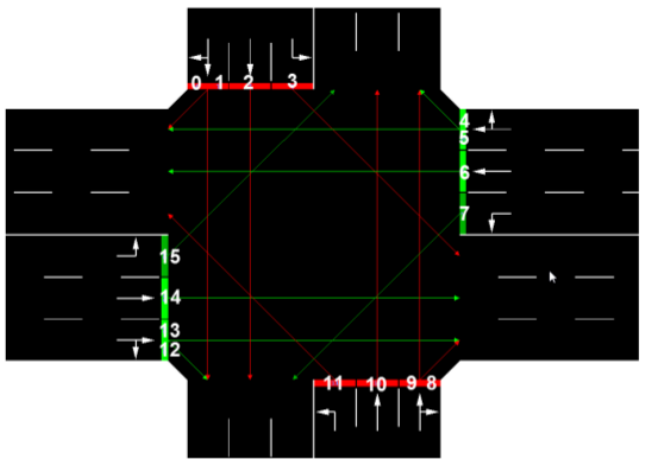

(a) Signalled Four-way

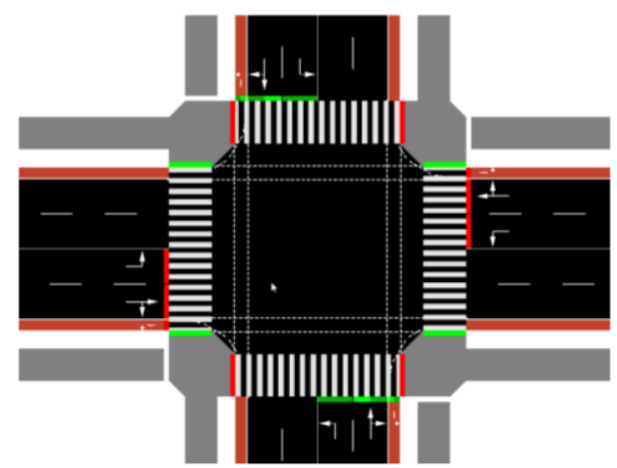

(b) Signalled Multi-modal four-way

Figure 4.3: Four-way junctions designed in SUMO

\subsubsection{Generating traffic demand}

The traffic demand file (.rou.xml) is uded to generate traffic in SUMO. It must be noted that SUMO used the metric scale for representing speed in distance. Speed is represented in meters/sec and distance in meters. The minGap parameter, is the time to collision and is denoted in seconds. For example, passenger vehicle will be denoted as shown below: 


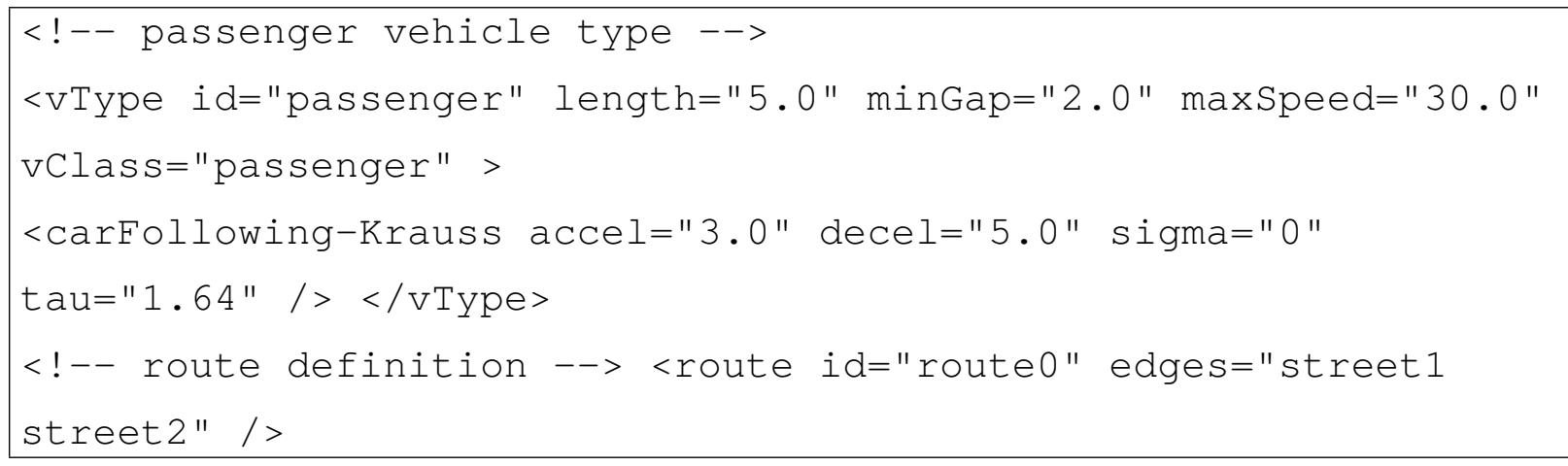

A passenger vehicle with a model defined above will have a maximum speed of $30 \mathrm{~m} / \mathrm{s}$ and maintain a minimum gap of 2 seconds to the leading vehicle before applying breaks. It follows a Krauss car following model with user-defined parameters. Alternatively, a vehicle trip may be created by defining the origin and the destination edges as shown below. The routing engine calculates and assigns the shortest path to the vehicle. Intermediate nodes can be assigned using the via parameter.

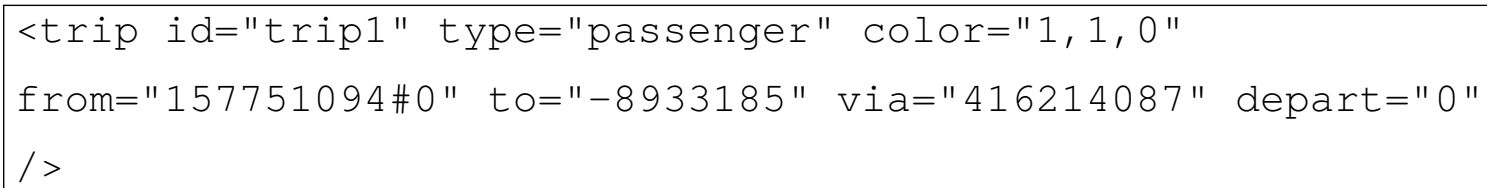

A vehicle flow consisting of multiple vehicles on the same starting and ending edge can also be created. Vehicles in a flow have the same vehicle type but unique ID to identify each vehicle. The rate of vehicle flow can be defined by either specifying the vehicles Per Hour or the probability of emitting a vehicle every second.

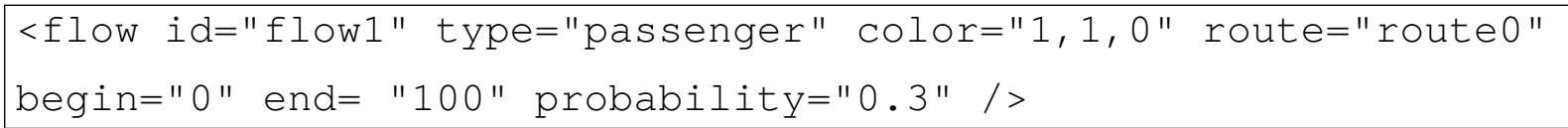

Figure 4.4 shows a four way junction with multiple vehicle flows. Vehicles of the same color belong to the same flow.

At this point we have a network of roads and traffic flow. All vehicles on the road have DSRC radios for wireless communication. In the following section we will see how V2X communication can be setup using $\mathrm{OMNeT}++$. 


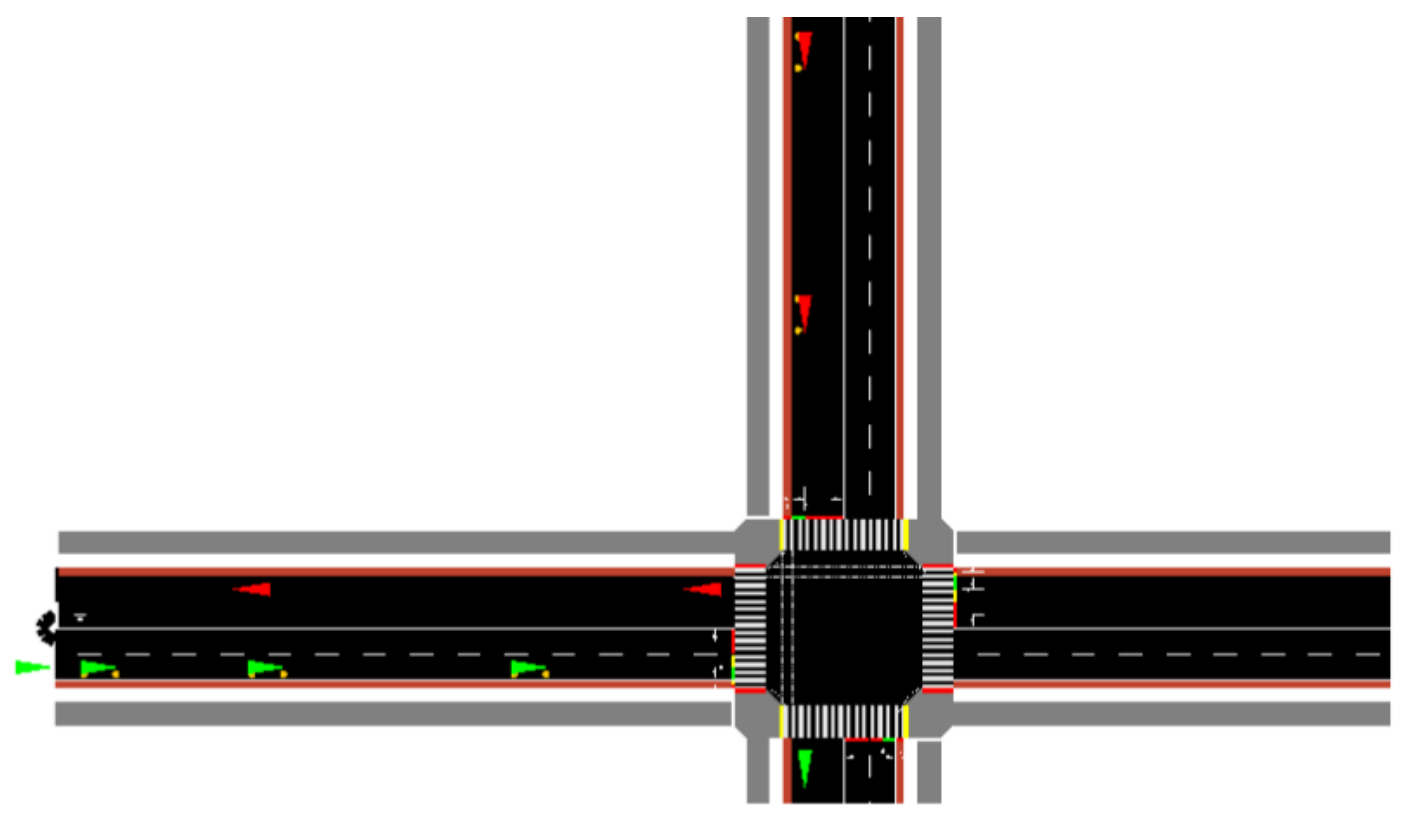

Figure 4.4: A junction with two vehicle flows

\subsection{V2V Communication in VENTOS}

In VENTOS vehicles can communicate with other vehicles using on-board DSRC radios. The radios follow the IEEE $802.11 p$ wireless standards. The standard has two tpes of channels : (i) 1 Control Channel (CCH) and (ii) 6 Service Channels (SCH). Figure 4.5 shows the channel distribution. $\mathrm{CCH}$ is used to broadcast control messages where as $\mathrm{SCH}$ is used for non-safety data transfer. Each of the channels has a bandwidth of 20-MHz. RoAdNet transmit all messages over the $\mathrm{SCH}$.

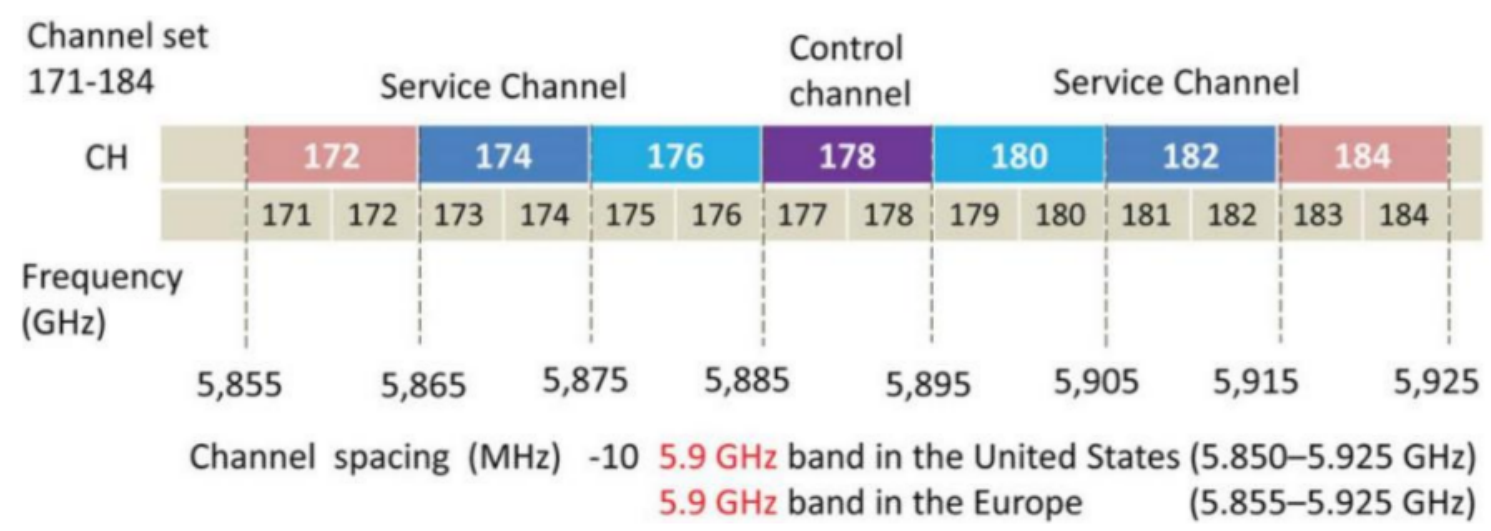

Figure 4.5: Channel distribution for $\mathrm{CCH}$ and $\mathrm{SCH}$ 


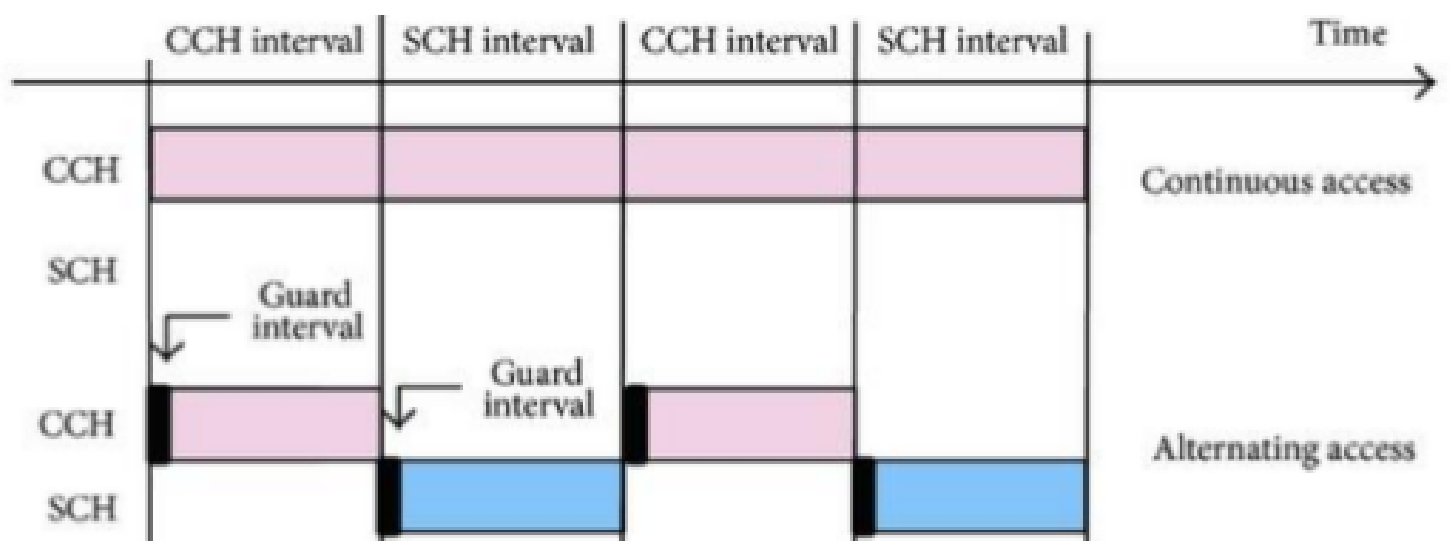

Figure 4.6: Timing diagram for $\mathrm{CCH}$ and $\mathrm{SCH}$

The IEEE 1609.4 provides enhancements to the IEEE 802.11 MAC to support WAVE operations. VENTOS provides Continuous Access and Alternating Access schemes. It can be seen in Figure 4.6, that in continuous access mode, the channel stays tuned to $\mathrm{CCH}$ all the time. In alternate access mode the channel alternates between $\mathrm{CCH}$ and $\mathrm{SCH}$ interval. One of the six SCH channels is chosen, as negotiated during the $\mathrm{CCH}$ interval.

The MAC layer also implements an EDCA (Enhanced Distributed Channel Access) mechanism for sharing the channel. There are separate EDCA ques for $\mathrm{CCH}$ and SCH. By using EDCA high priority data is sent faster than low priority data.

\subsection{Scenario Design for Testing RoAdNet}

The vehicular network is modeled as a long stretch of highway $(10000 \mathrm{~m})$ with multiple lanes and and multiple traffic flows in all possible lanes and directions. Different vehicle densities are considered to replicate free flowing, medium congestion and high congestion scenarios. This is achieved by varying the vehicle insertion rate and the maximum vehicle speed in the simulation. Let $\delta$ be the desired density in the region (or the number of vehicles per unit area at any given time) and $R$ denote the maximum radio range for each vehicle. The unit area is the region covered by the communication range of a single vehicle i.e. $\pi R^{2}$. For a 4 lane highway, with 2 lanes in each direction the desired vehicle density in each lane $\delta_{L}$ is $\delta / 4$. Let $G$ denote the gap between two consecutive vehicles. Thus $G=R / \delta_{L}$ is the desired separation between the vehicles. For a given insertion rate of $I \mathrm{~Hz}$, the maximum 
speed the vehicle $V_{(\max )}$ will be $I * G$. 


\section{Chapter 5}

\section{Results}

The chapter presents the results and discussion of multiple aspects of RoAdNet's design and its overall performance evaluation. The first part focuses on the comparison of distance sensitive and non-distance sensitive forwarding approaches. Then we discuss about the trade-offs of using different radio ranges for communication and select the best range for this application. The various optimization parameters in the protocol are then tested to find a suitable working combination. The performance of multi-resolution forwarding is then discussed. Then, the overall performance of RoAdNet is discussed. The last part presents a real-time application showing how availability of information in advance can affect the energy efficiency of a vehicle.

The four performance matrices that are used to analyze RoAdNet are:

1. Staleness (S): Staleness defines the the quality of information from a node. It says how old or recent the current information about a particular node is.

2. Packet Delivery Ratio (PDR): It is the ratio of number of packets delivered to the number of packets transmitted in the network.

3. Distance Estimation Error Ratio (DEER): DEER is the the ratio of error in distance estimation to the actual distance

4. Latency (L): Latency is the time between origin and reception of a message.

Details about the performance metrics can be found in Chapter 1. 


\subsection{Distance Sensitive vs Non Distance Sensitive For- warding}

In Chapter 3, we discussed about the differences between the distance sensitive (DS) and non distance sensitive (NDS) approaches of forwarding messages in a multi-hop network. Now we will compare the performance of these approaches based on the three performance metrics discussed in Chapter 1, i.e staleness, packet delivery ratio and distance estimation error ratio. The results presented are for a radio range on $500 \mathrm{~m}$ at a speed of $12 \mathrm{~m} / \mathrm{s}$. The results for other radio ranges and speeds show a similar trend. Also, the base transmission frequency and forwarding frequency are $1 \mathrm{~Hz}$. Multiple traffic flow densities were considered. Low, medium and High density correspond to 25,50 and 75 vehicles/1000m.

\subsubsection{Effect on Staleness}

Figure 5.1, shows the effect of distance sensitivity on staleness for different traffic densities. In Figure 5.1a, it can be seen that at low density, DS maintains the staleness within the 1-hop range. Beyond 1-hop range, it is higher than NDS. For medium and high density as shown in Figure 5.1b and 5.1c, DS significantly reduces the staleness at smaller distances. 


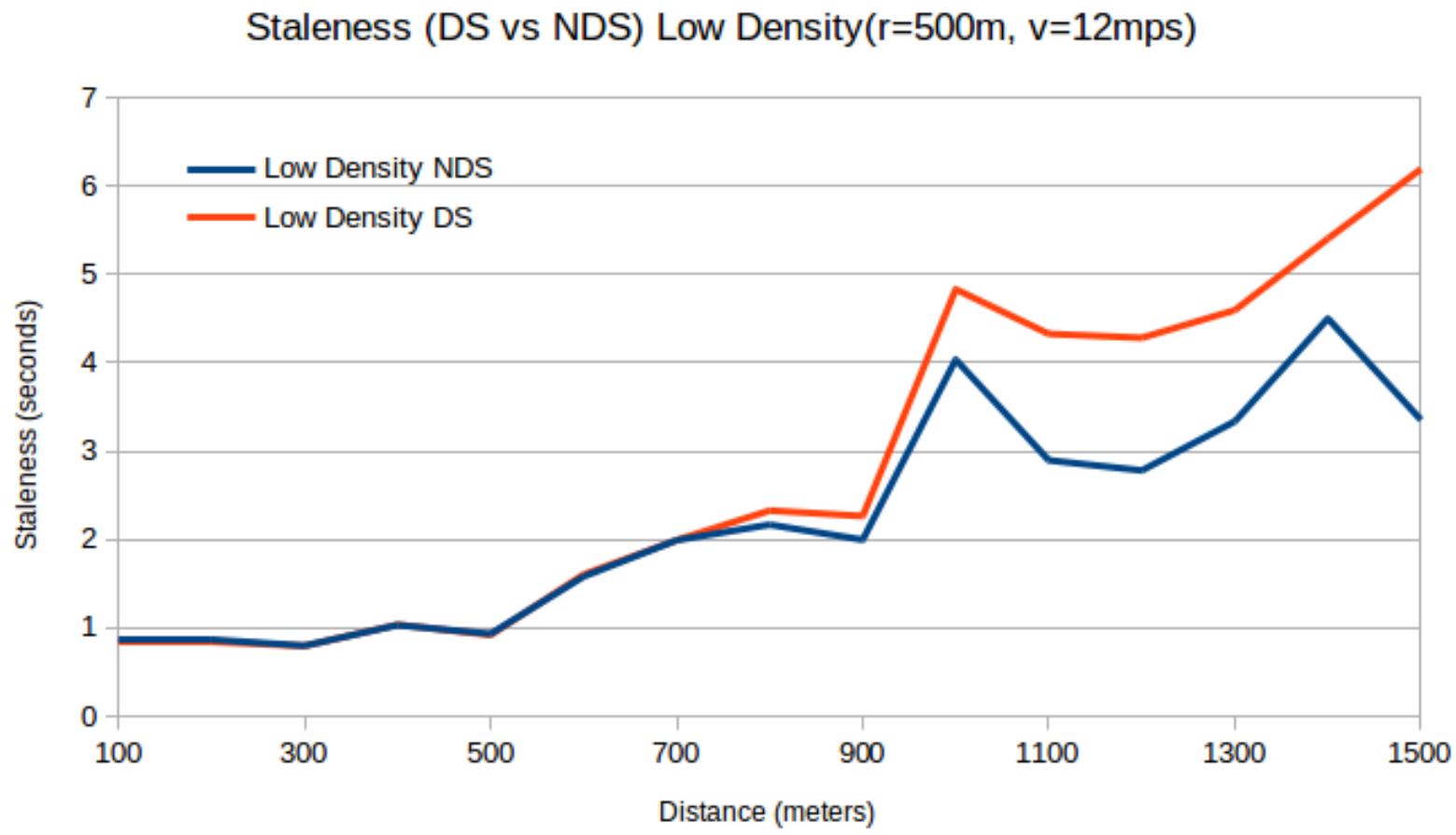

(a) Low Density

Staleness (DS vs NDS) Medium Density $(r=500 m, v=12 \mathrm{mps})$

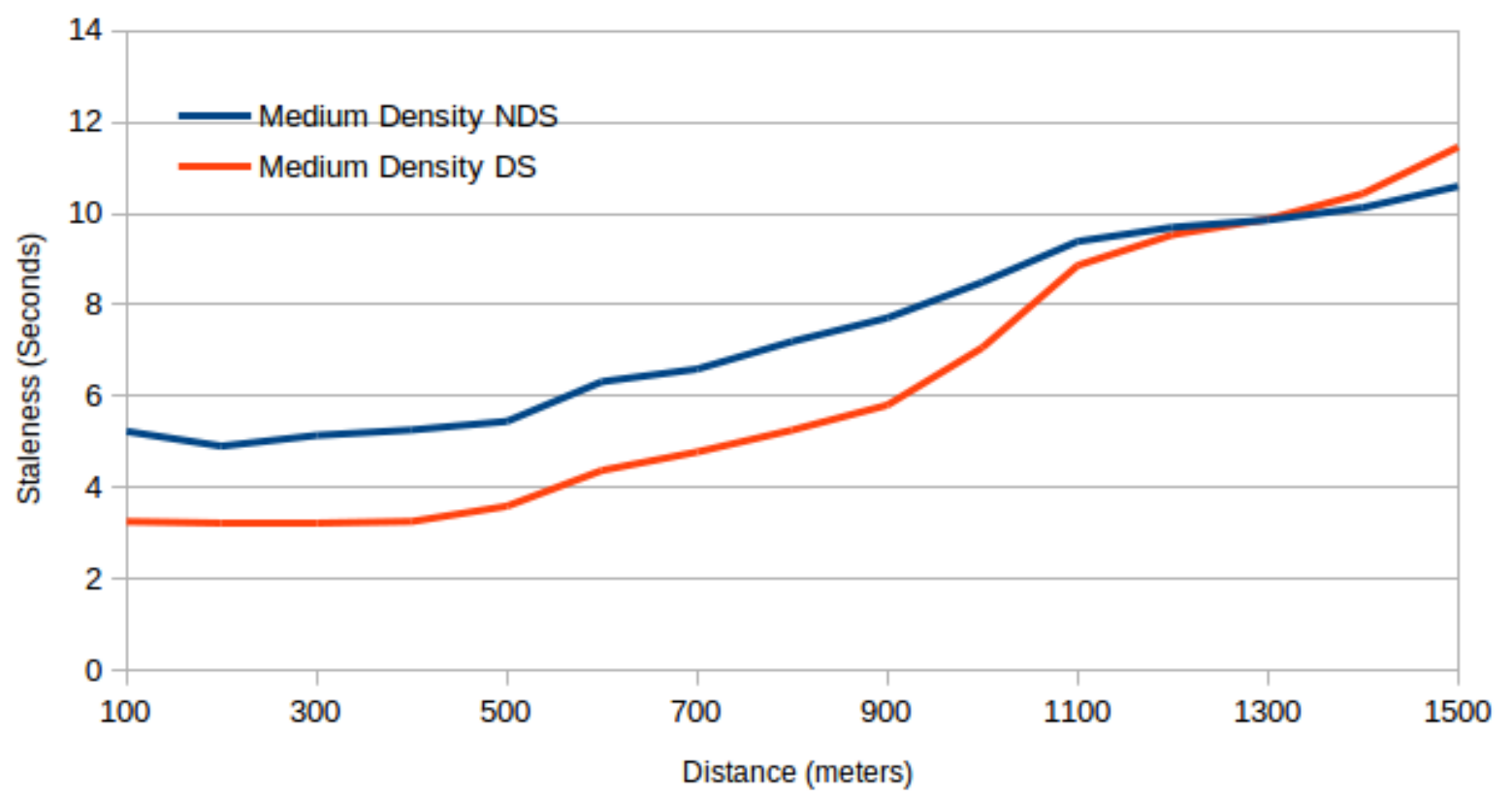

(b) Medium density

taleness at different density of traffic flow 


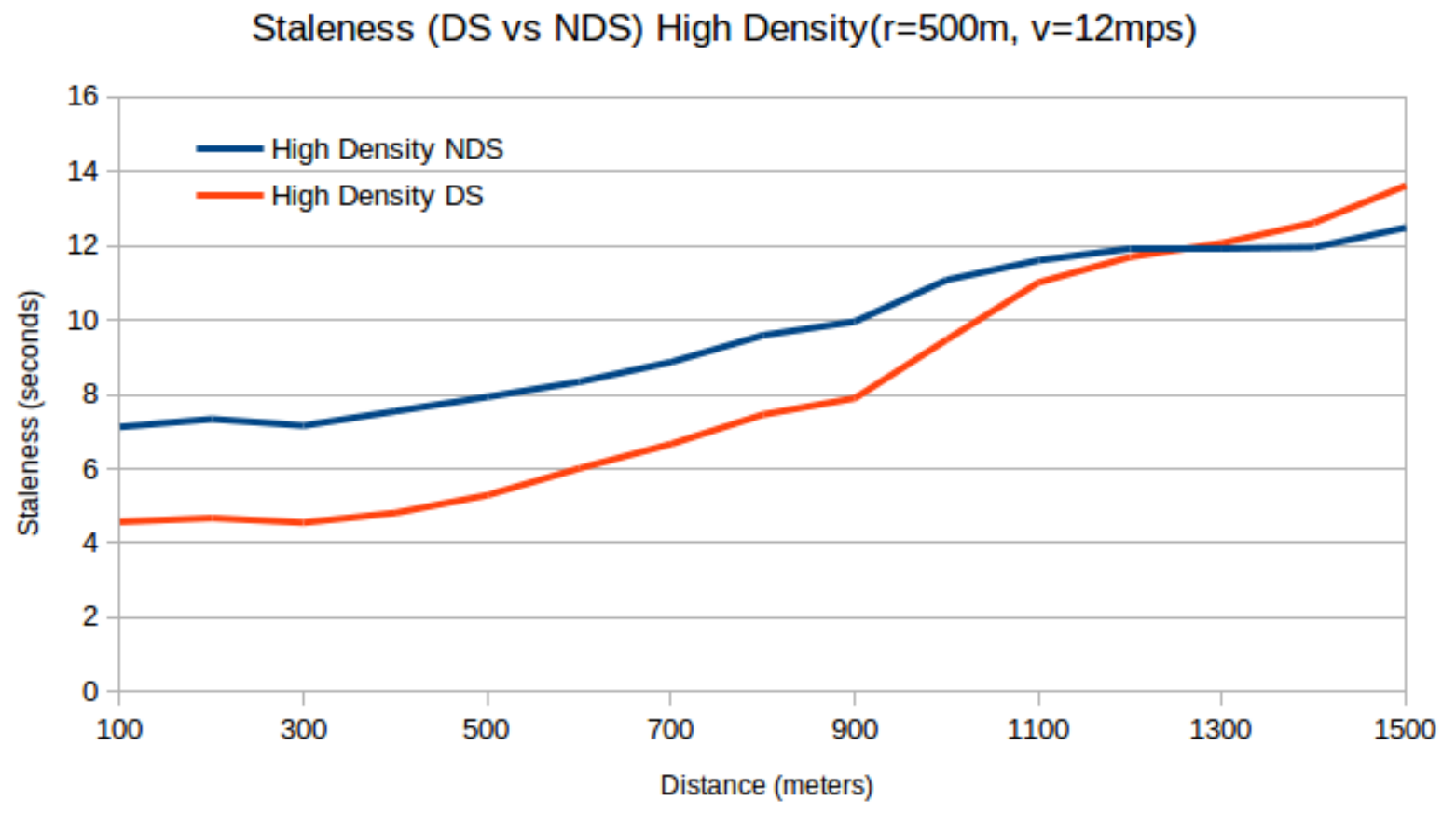

(c) High Density

Figure 5.1: Effect of distance sensitivity on staleness for different densities

\subsubsection{Effect on Distance Estimation Error}

Figure 5.2, shows the variation in distance estimation when using DS and NDS forwarding approaches. It can be seen that at low density (Figure 5.2a, the error in maintained by DS up to the second hop after which a marginal increase in seen. At medium and high densities, Figure $5.2 \mathrm{~b}$ and $5.2 \mathrm{c}$, a significant improvement is error is observed, specially within the 1-hop range.

\subsubsection{Effect on Packet Delivery Ratio}

Figure 5.3, shows the packet delivery ratios for different communication ranges and at different densities. Distance sensitive forwarding out performs non distance irrespective of density. 
Distance Error Ratio (DS vs NDS) Low Density( $r=500 m$, v=12mps)

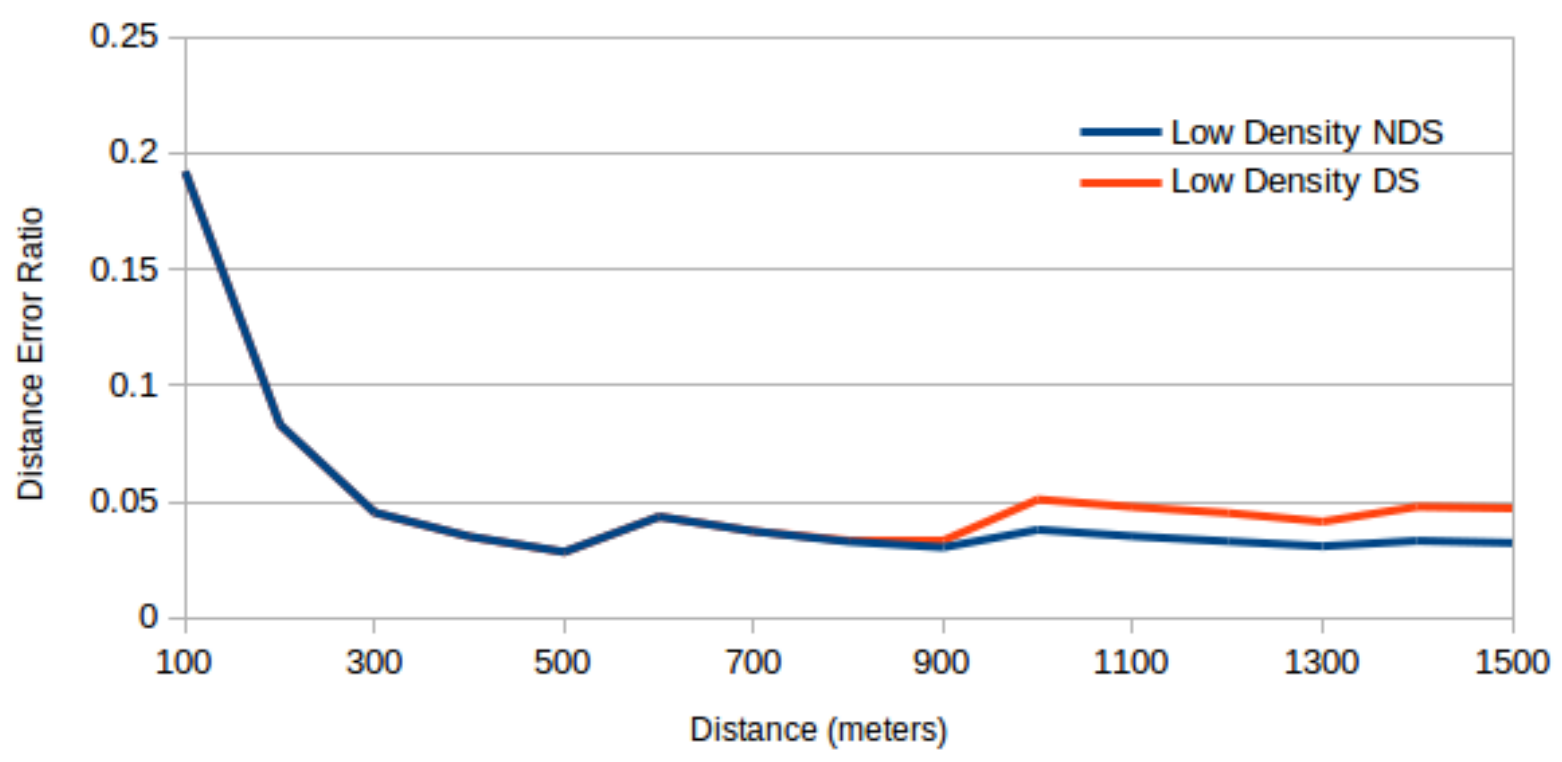

(a) Low Density

\section{Distance Error Ratio (DS vs NDS) Medium Density(r=500m, v=12mps)}

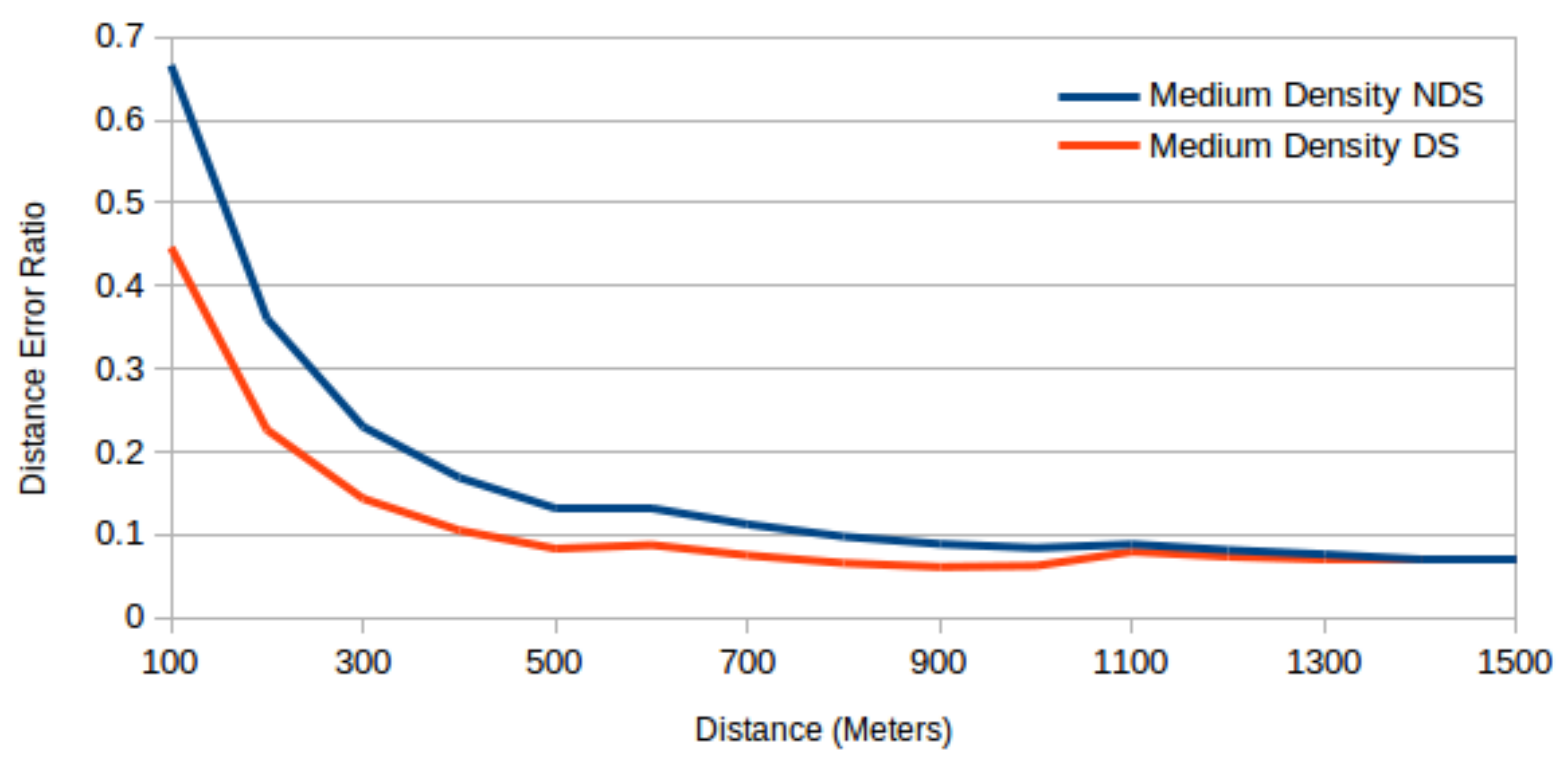

(b) Medium density

\subsubsection{Discussion}

It can seen that distance sensitive approach performs better than non-distance sensitive approach for all the three parameters. This is because DS suppresses a lot of information 


\section{Distance Error Ratio (DS vs NDS) High Density(r=500m, v=12mps)}

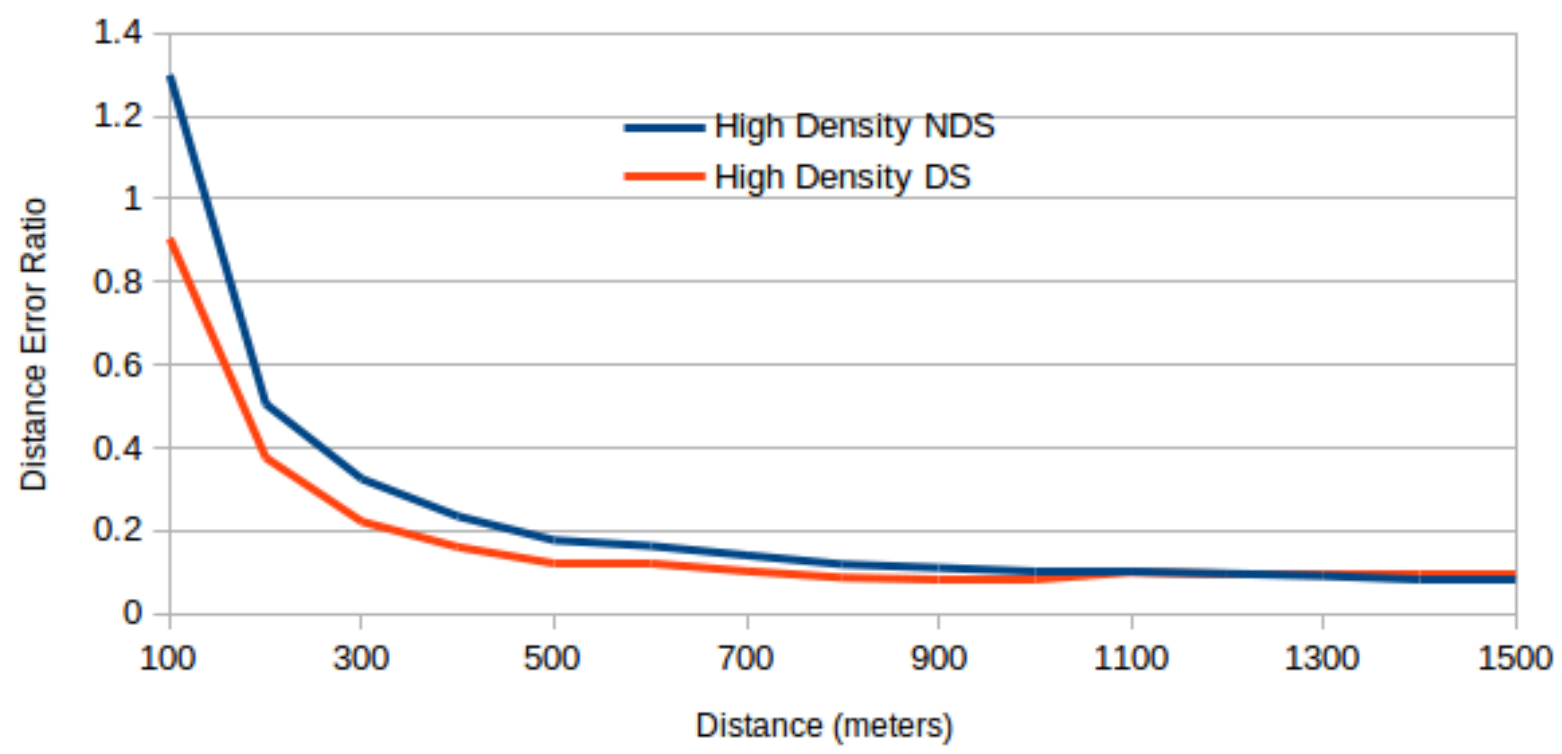

(c) High Density

Figure 5.2: Effect of distance sensitivity on Distance Estimation Error for different densities

based on distance and those packets do not go out to the network. This frees up network bandwidth, resulting in higher throughput and effective bandwidth usage. Delivery of more packets, corresponds to more frequent information about a node, which results lower staleness and better distance estimation.

The results support our analogy that information from longer distances can be transmitted sparsely. We are able to do so because as we move away from the point of origin on the message the more time a vehicle has to react to the situation. This will enable the use of V2V information for applications like highway merging and rerouting.

\subsection{Study of variation in radio range}

The choice of radio range is critical to the working of RoAdNet. A very small range would result in large number of hops to reach longer distances where as a long radio range can have too many nodes within communication range resulting in network congestion. This section 


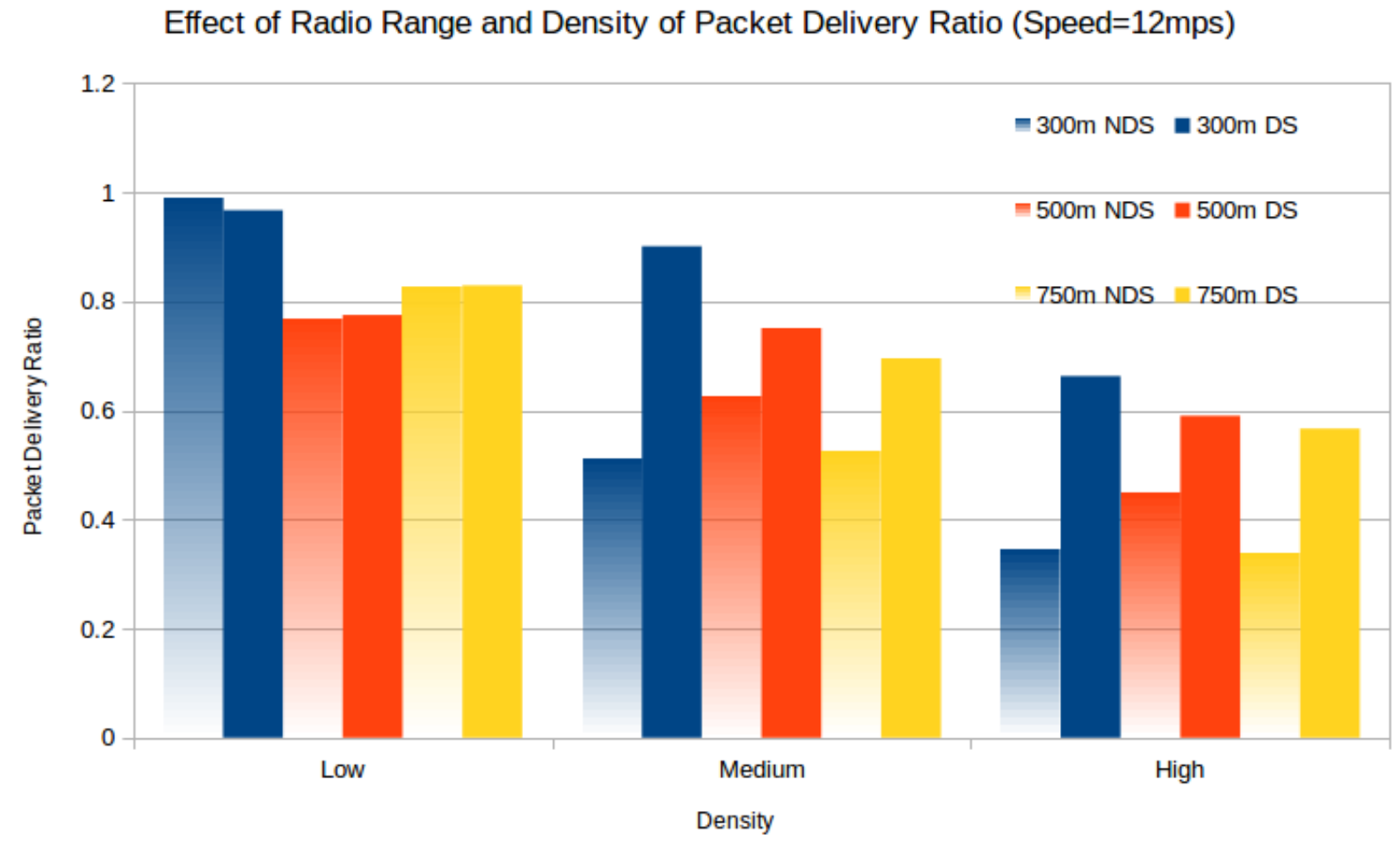

Figure 5.3: Effect of distance sensitivity on Packet Delivery Ratio

compares the performance of three different communication ranges, i.e. $300 \mathrm{~m}, 500 \mathrm{~m}$ and $750 \mathrm{~m}$ based on staleness, distance estimation error and packet delivery ratio. Additionally, we take a look at the number of packets per bin to get some useful insights about their effect.

\subsubsection{Effect on Staleness}

Figure 5.4, shows the variation in staleness for different communication ranges for both distance sensitive and on distance sensitive approaches. Smaller communication ranges result in lower staleness within the first hop but as the number of hops increases the staleness increases rapidly. On the other hand large communication range has very high staleness within the first hop, which is undesirable for safety critical applications. A medium communication range, $500 \mathrm{~m}$, blends the performance of the short and long ranges very well. It has acceptable latency within a single hop and has the least latency at $1500 \mathrm{~m}$. 


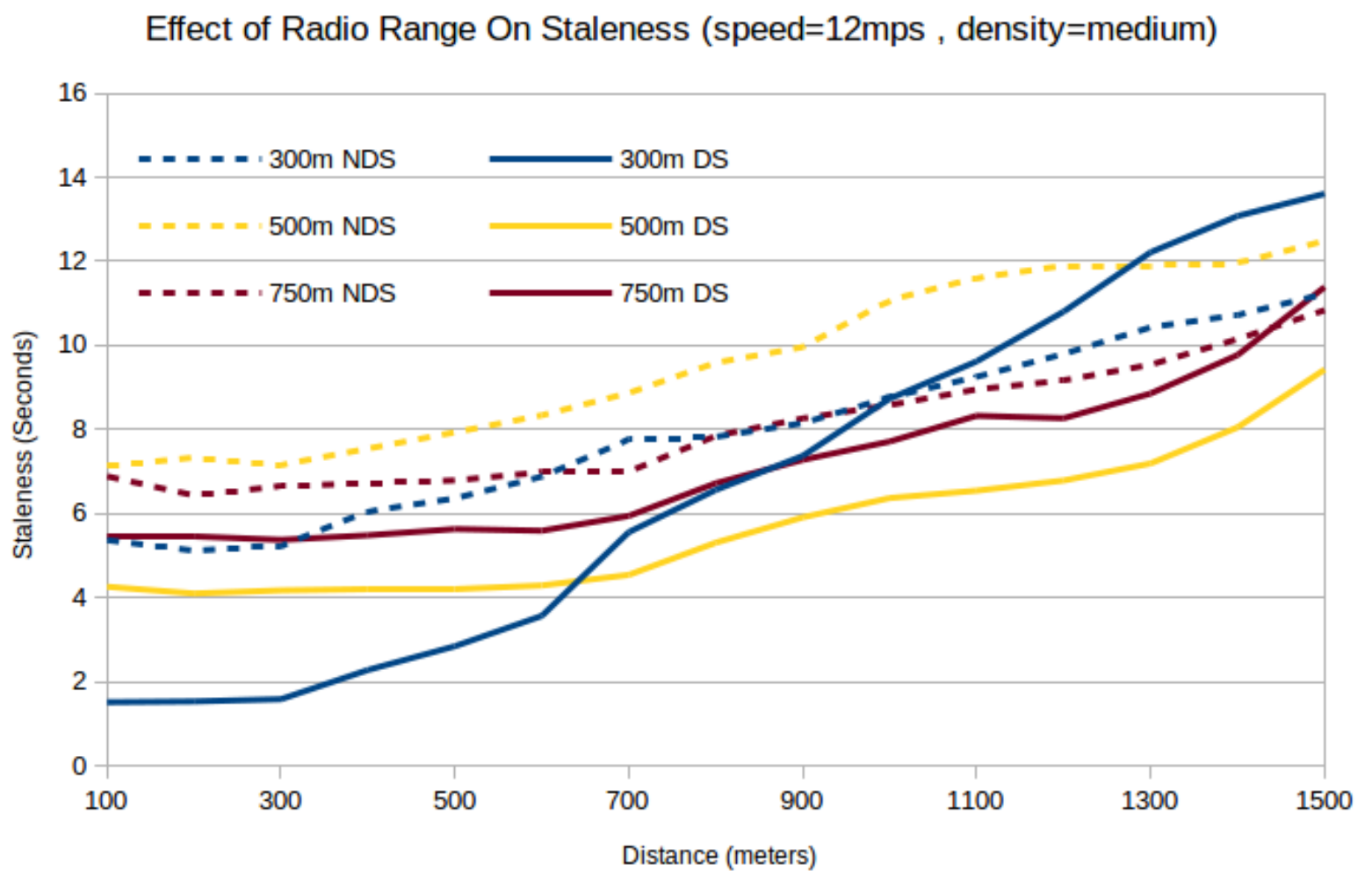

Figure 5.4: Effect of radio range on staleness

\subsubsection{Effect on distance error estimation}

It can be seen in Figure 5.5 that long range has the maximum error where as short range has the minimum error in distance estimation, within the one hop range. At longer distances the performance is similar for differnt radio ranges.

\subsubsection{Effect on packet delivery ratio}

It was expected that a small radio range will have higher deliver ratio as the number of nodes within the range will be less. Figure 5.6 shows approval of the same.

\subsubsection{Effect on number of packets delivered}

It is critical to have enough packets at the end of $1500 \mathrm{~m}$ to enable sufficient information delivery at $10000 \mathrm{~m}$. To analyze this aspect, we take a look at an extra parameter, the 


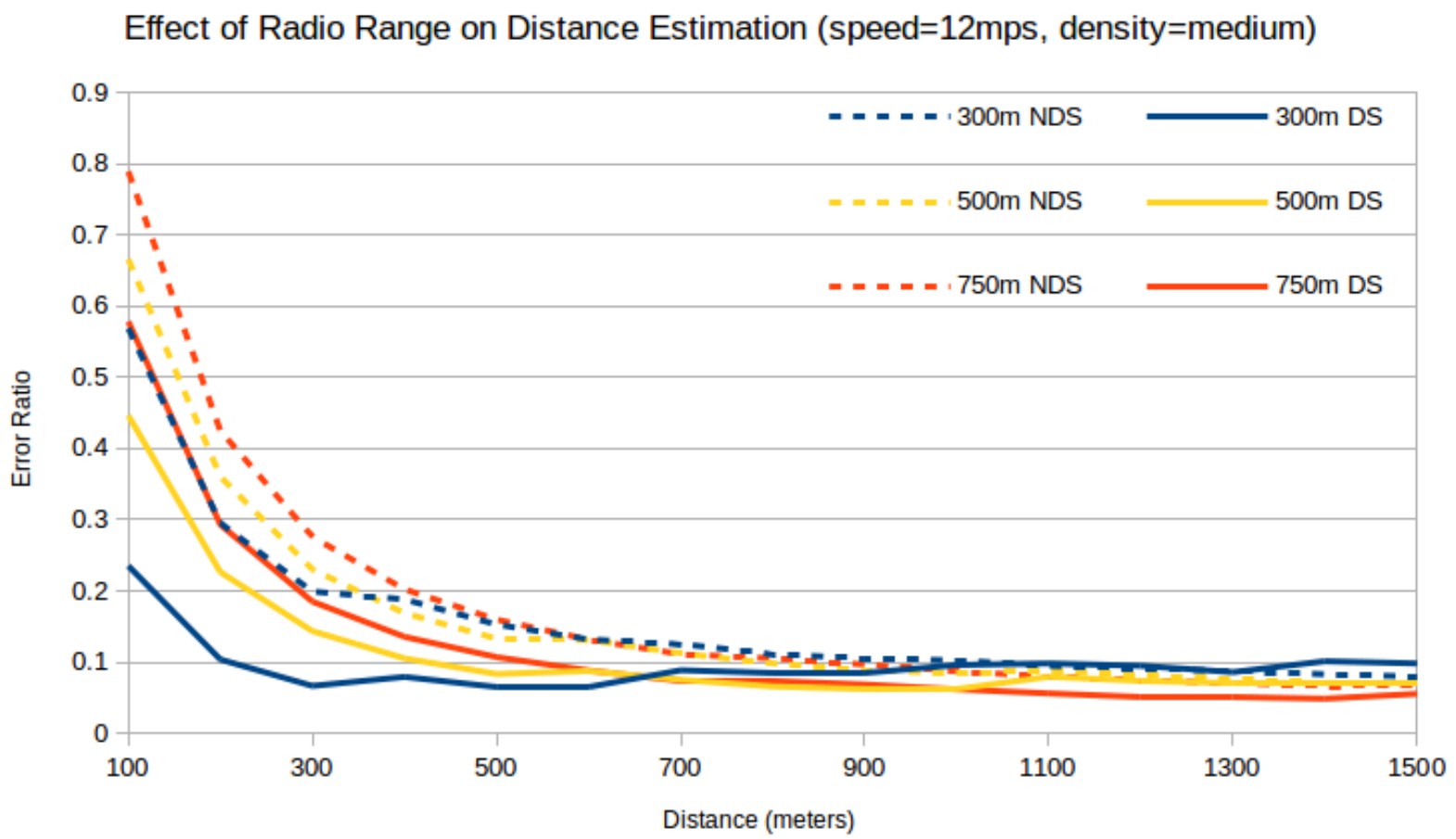

Figure 5.5: Effect of radio range on distance estimation error

number of packets delivered at a given distance. It can be seen in Figure 5.7 that long radio range delivers most packets at $1500 \mathrm{~m}$ and short radio range barely manages to deliver any.

\subsubsection{Discussion}

Discussions earlier in this section show that short and long communication ranges have their own advantages and disadvantages. While small radio ranges have superior staleness and packet delivery ratios, they simply do not deliver enough packets over multiple hops. Long communication range looks more promising for delivering packets at very long distances.

For the rest of the discussion we choose a medium communication range of 500 meters with Distance Sensitivity, as it inherits certain desirable properties of both short and long range radios. The reliability of the RoAdNet can be further improved by tuning various parameters in the protocol. 


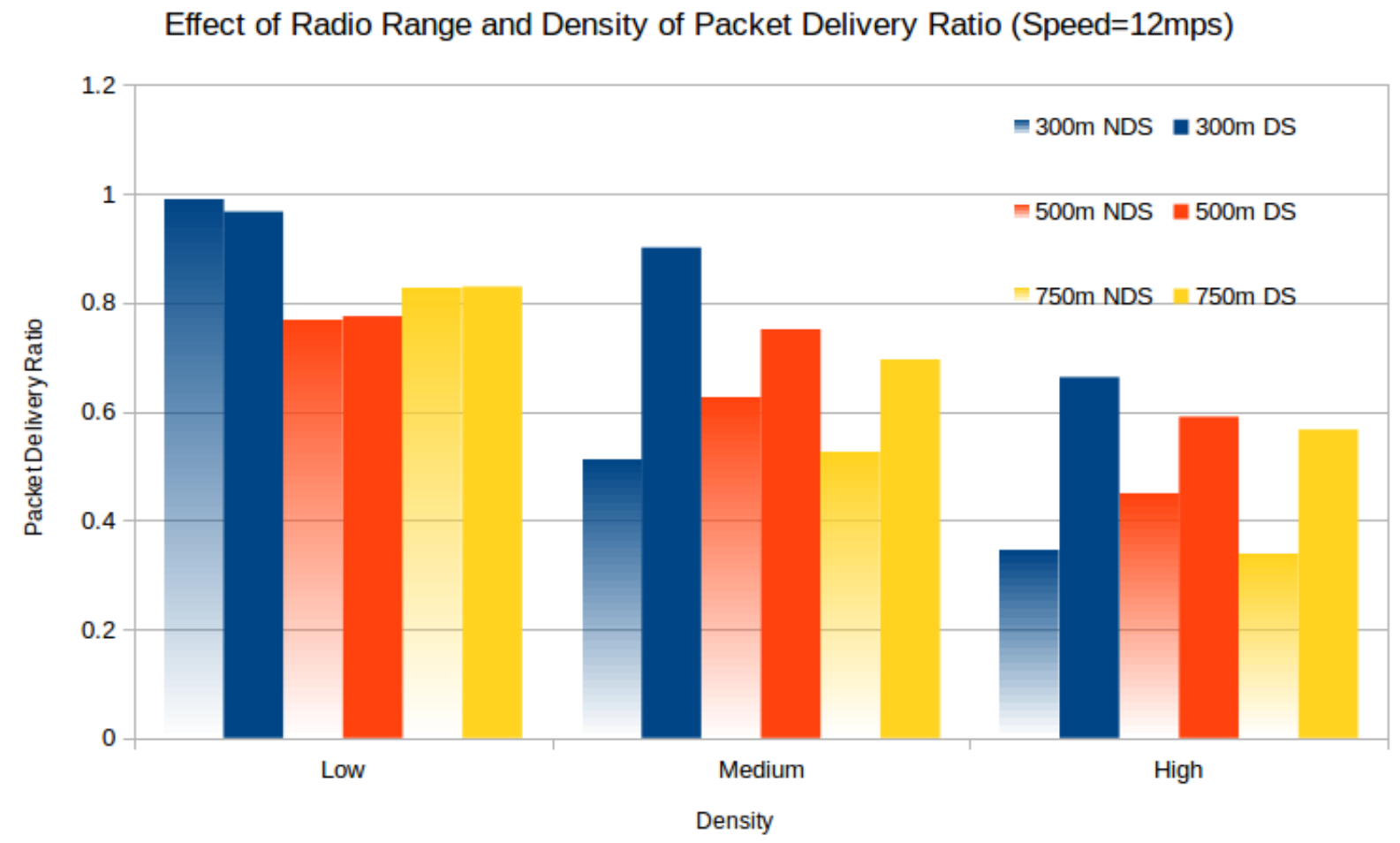

Figure 5.6: Effect of radio range on packet delivery ratio

\subsection{Parameter optimization in RoAdNet}

In this section we will see the effect of Suppression Threshold and the EDCA/Transmissoin Queue Length on various network parameters.

\subsubsection{Suppression Threshold}

\begin{tabular}{|c|c|l|}
\hline Threshold & Packet Delivery Ratio & Staleness \\
\hline \hline 0 & 0.4 & High \\
\hline 1 & 0.74 & Moderate \\
\hline 2 & 0.6 & Least \\
\hline
\end{tabular}

Table 5.1: Network performance for different thresholds

Figure 5.8 and Figure 5.9 show the variation in packet deliver ratio and staleness for different values of suppressing threshold. $t h=0$, implies no packets were suppressed. Table 


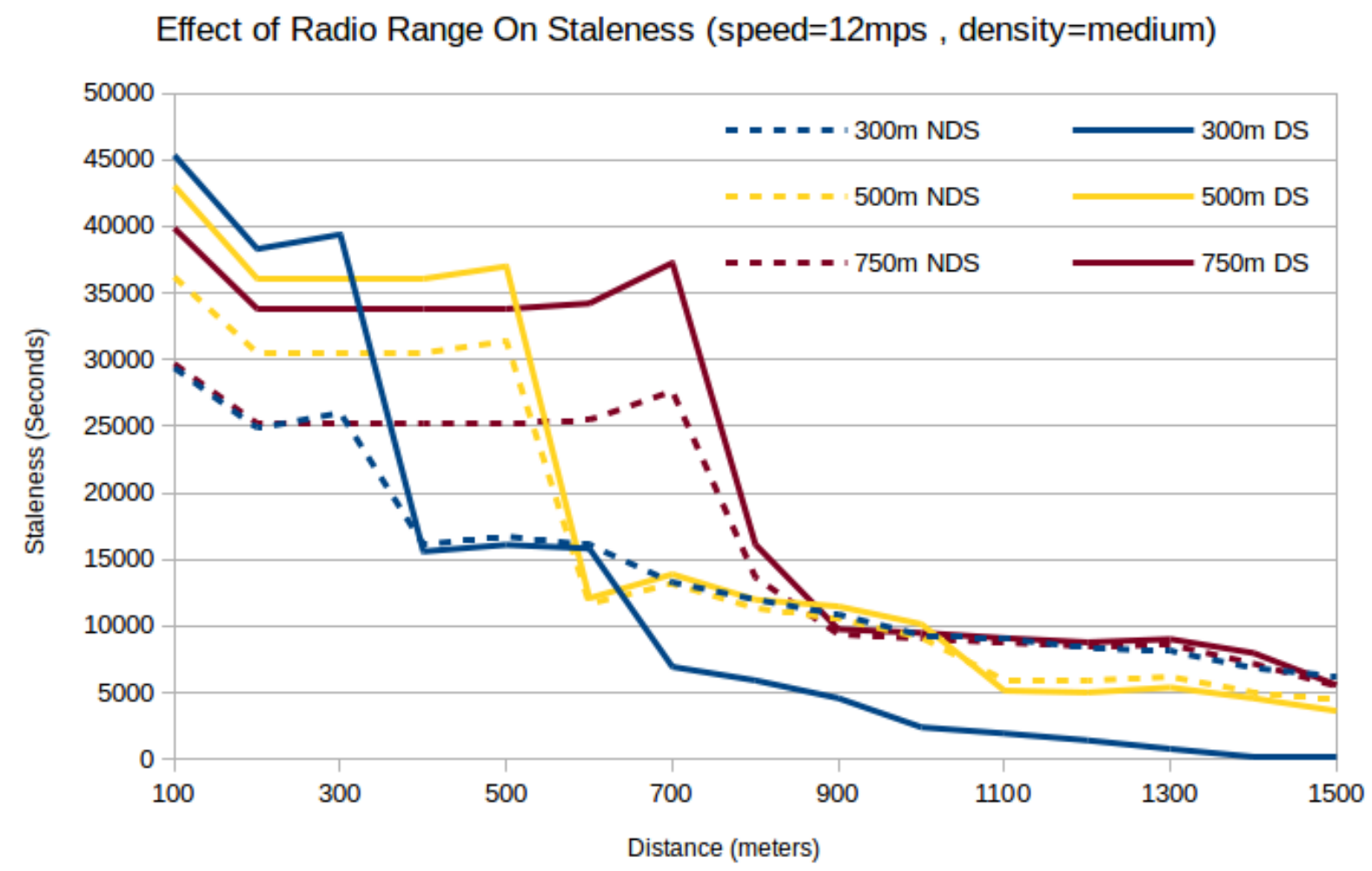

Figure 5.7: Effect of radio range on packets delivered per bin

5.1 summarizes the effect of suppression threshold on various network parameters. The selection of threshold is a trade off between packet deliver ratio and staleness.

\subsubsection{Transmission Queue Length}

All the packets that are to be send out of a node are queued up in the transmission queue. A longer queue can increase the staleness in the network as the packets will have to wait longer before they get out of the queue, where as a smaller queue will result in packets being dropped at the node itself. A queue length of Zero(0) implies an infinite queue.

It is seen in Figure 5.10, that an infinite queue results in higher delivery ratios. Lower queue sizes have lower delivery ratios but staleness is also decreased, as seen in Figure 5.11. With increasing queue size delivery ratio increases ultimately approaching the numbers of an infinite queue. Also, a look a the number of packets delivered at long distances suggests that more packets are delivered for a medium queue size. This is essential when aggregate 
Effect of Suppression Threshold on Packet Delivery Ratio $(500 \mathrm{~m}$, speed $=12 \mathrm{mps}$, density=medium $)$

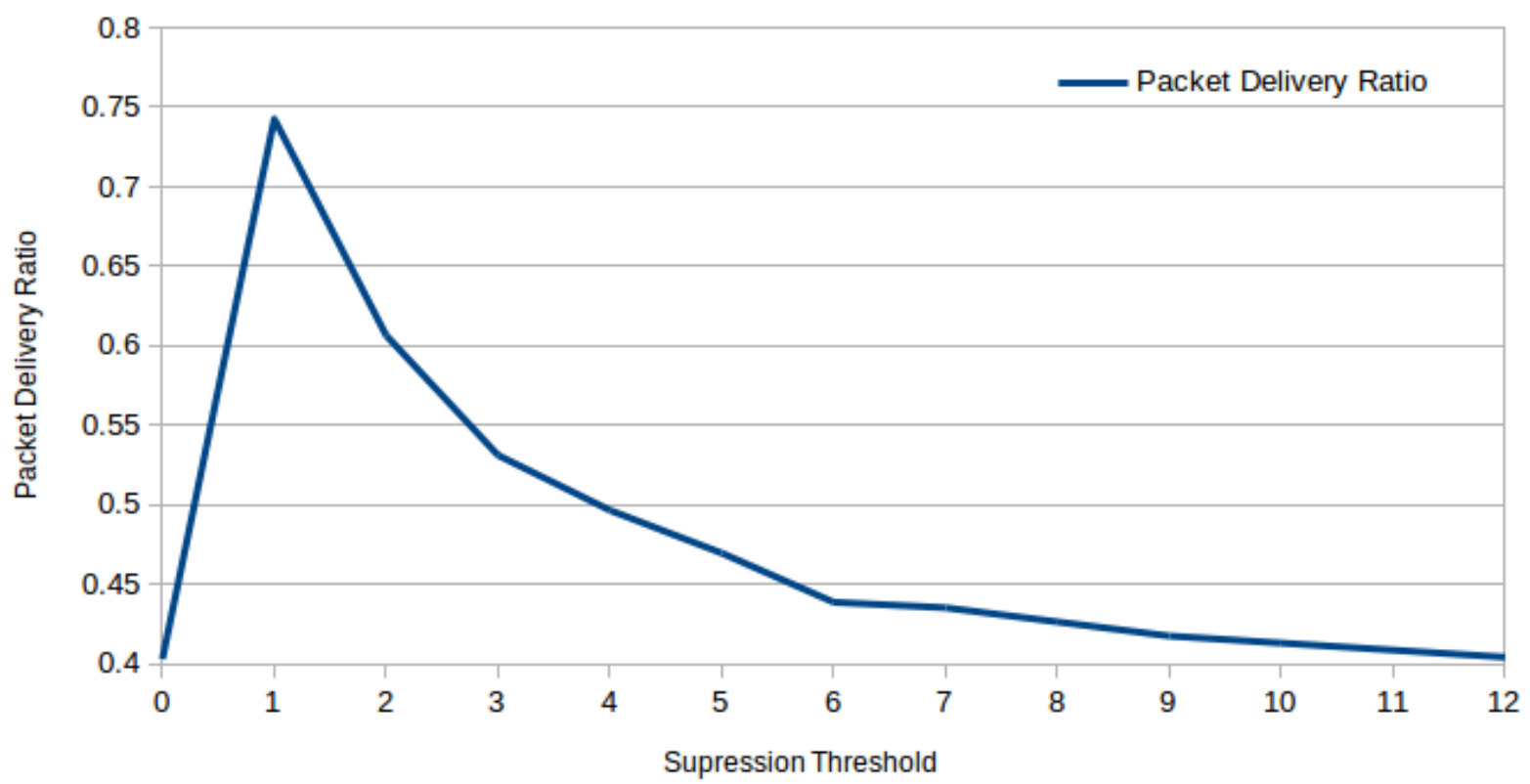

Figure 5.8: Effect of suppression threshold on packet delivery ratio

Effect of Supression Threshold on Staleness $(r=500 \mathrm{~m}-\mathrm{DS}$, density=medium, speed=12mps)

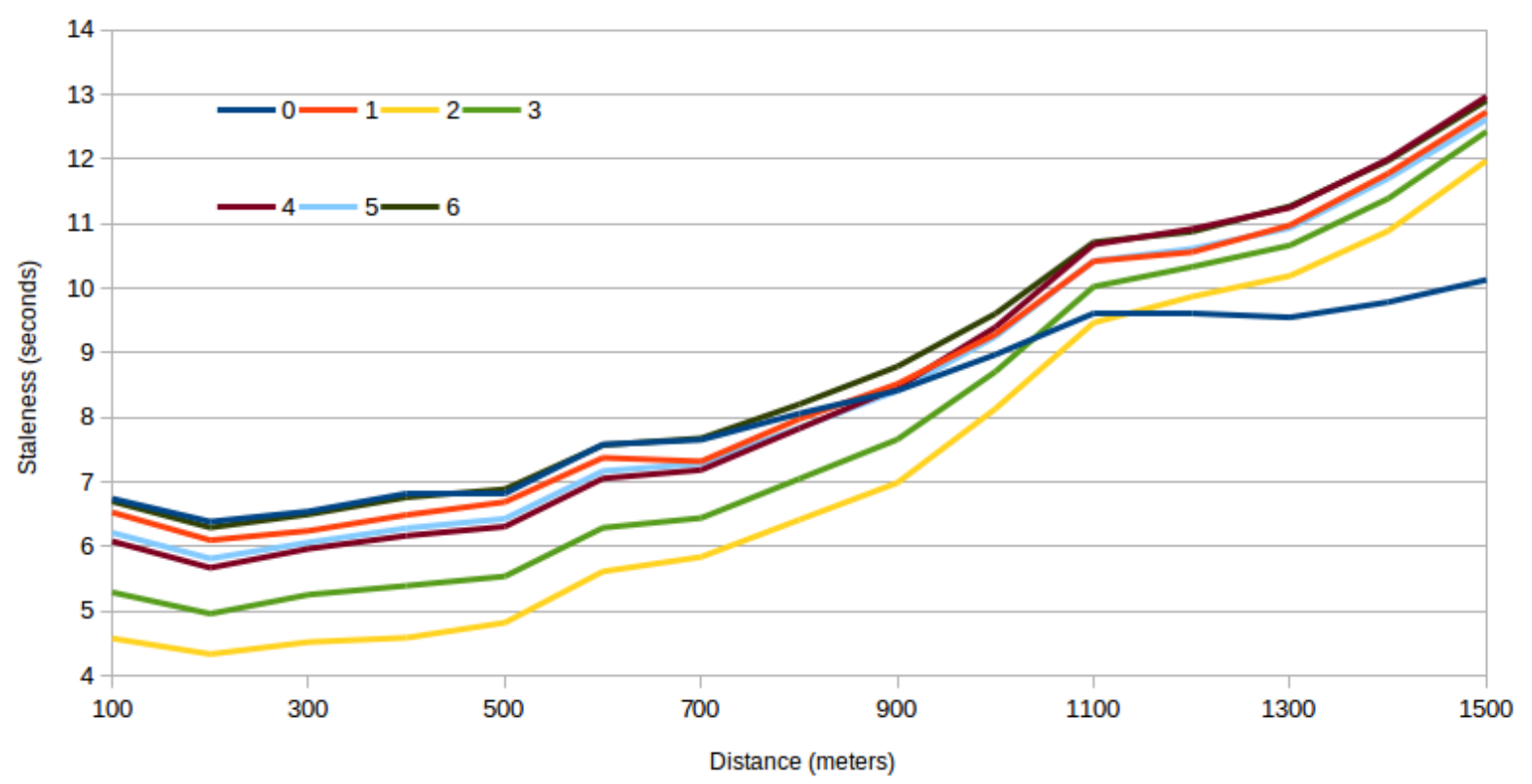

Figure 5.9: Effect of suppression threshold on staleness 


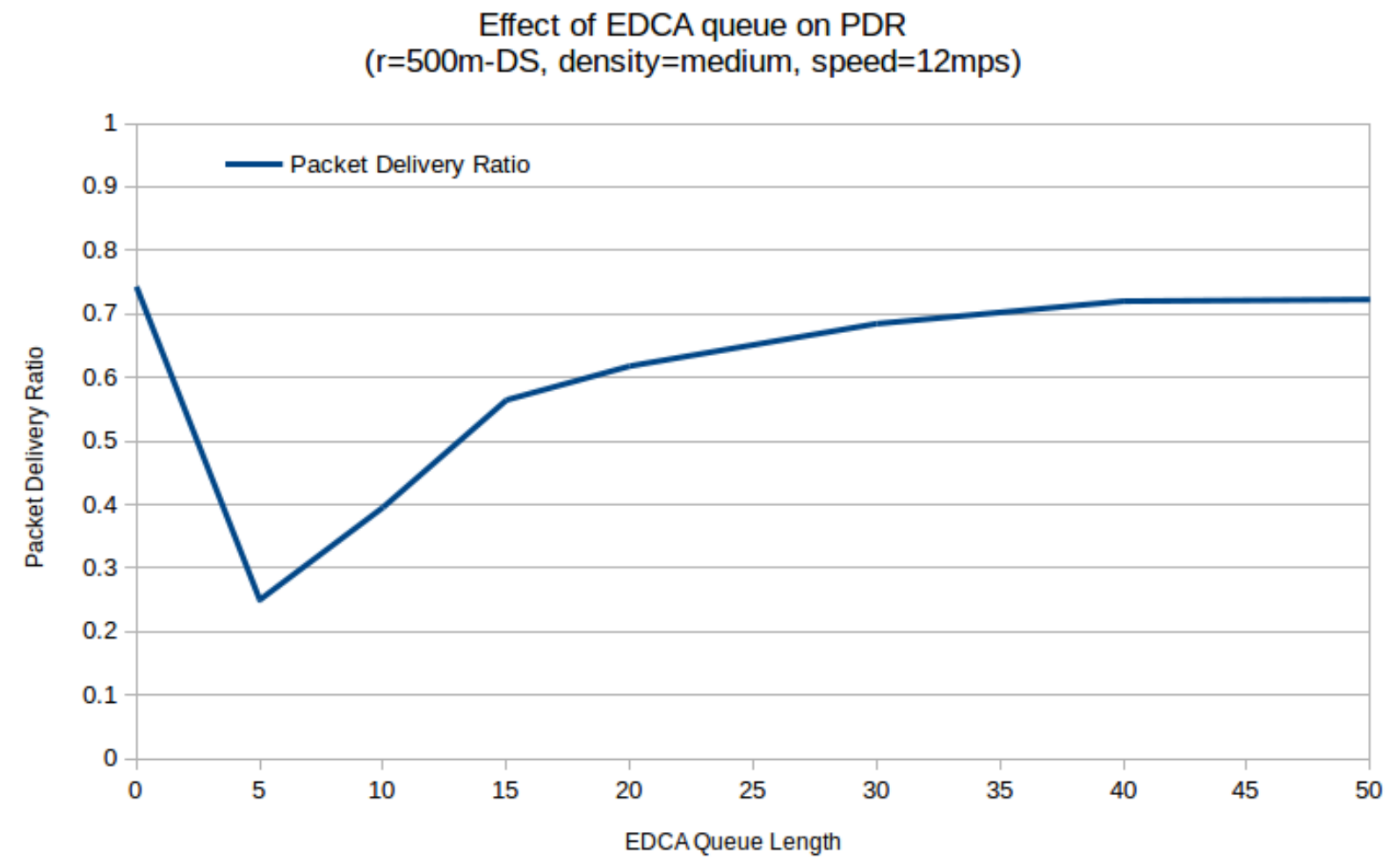

Figure 5.10: Effect of queue length on packet delivery ratio

packets need to be transmitted over long distances $(10000 \mathrm{~m})$. So the selection of queue length is a trade-off between latency and packet delivery ratio.

\subsection{Effect of multi-resolution transmission}

The results discussed so far were based on single resolution transmission. The rate of base transmission and forwarding were the same at $1 \mathrm{~Hz}$. In multi-resoltion transmission the base transmission frequency will be much higher at $5 \mathrm{~Hz}$ and the forwarding frequency is much lower. We consider $1 \mathrm{~Hz}, 0.5 \mathrm{~Hz}$, and $0.25 \mathrm{~Hz}$ forwarding frequencies along with the $5 \mathrm{~Hz}$ base transmission frequency. By doing this we expect to reduce the distance estimation error rate within the one hop range.

Figure 5.13 shows the effect of multiple resolutions, it can be seen that by increasing the base frequency the error in distance estimation at single hop improved significantly while maintaining low error rates at long distances. Further reduction in forwarding frequency 
Effect of EDCA queue on Staleness( $r=500 \mathrm{~m}-\mathrm{DS}$, density=medium, speed=12mps)

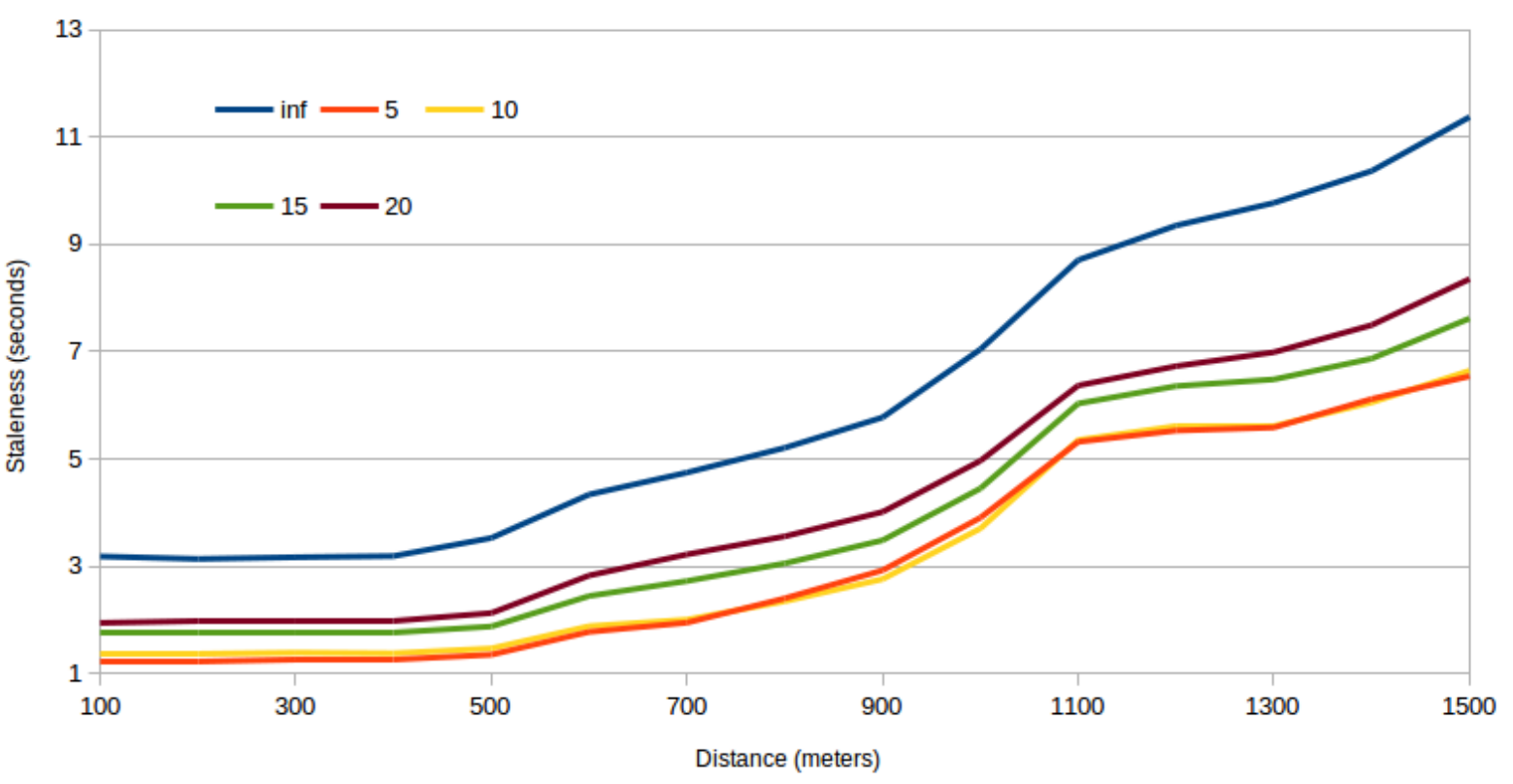

Figure 5.11: Effect of queue length on staleness

\section{Effect of EDCA Queue Size on Total Packets Delivered $(r=500 \mathrm{~m}$, speed $=12 \mathrm{mps}$, density=medium $)$}

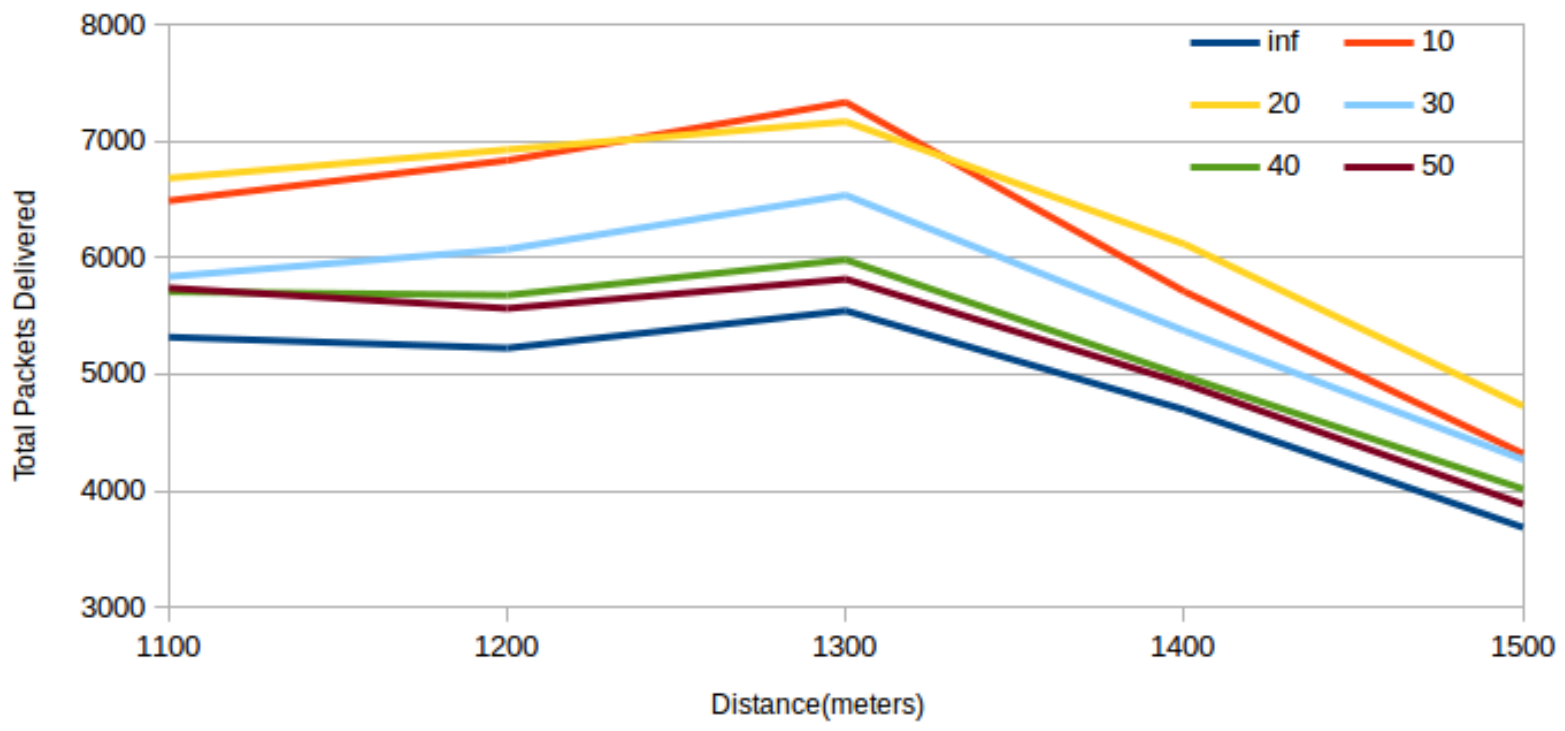

Figure 5.12: Packets delivery count at long distances 


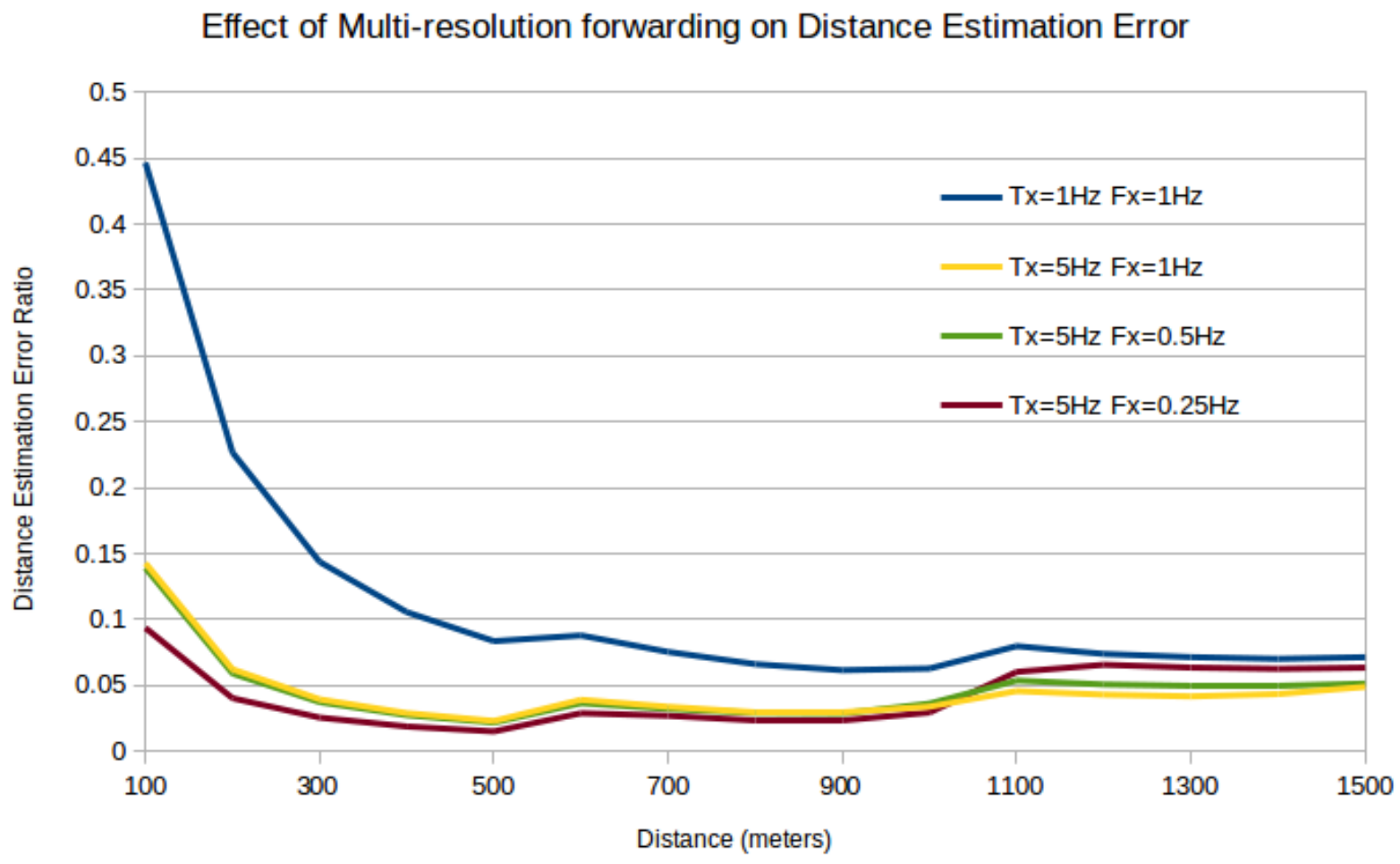

Figure 5.13: Effect of multi-resolution forwarding on distance estimation error

showed more improvement in the distance estimation. This can be attributed to the act that by increasing base frequency we expect to receive more information about vehicles in one hop. This also increases the network congestion increasing chances of collision and packet drops. This problem is countered by reducing the forwarding frequency, which results in higher bandwidth availability and better packet delivery rates within the single hop range.

\subsection{RoAdNet Overall Performance Evaluation}

\subsubsection{Effect of Distance Sensitive Forwarding}

Distance sensitive forwarding is expected to improve the staleness of messages within a single hop range, i.e. 500 meters communication range. Fig. 5.14 shows a box plot of staleness within the one hop range. It can be seen that DS forwarding results in lower staleness values in the network, including lower mean staleness and less outliers. This can be attributed to the fact the DS forwarding reduces the total number of packets being 
transmitted in the network. This is likely to result in lower packet collision, lesser packet drop and higher packet delivery rates. Hence, vehicles listen about other vehicles more frequently.

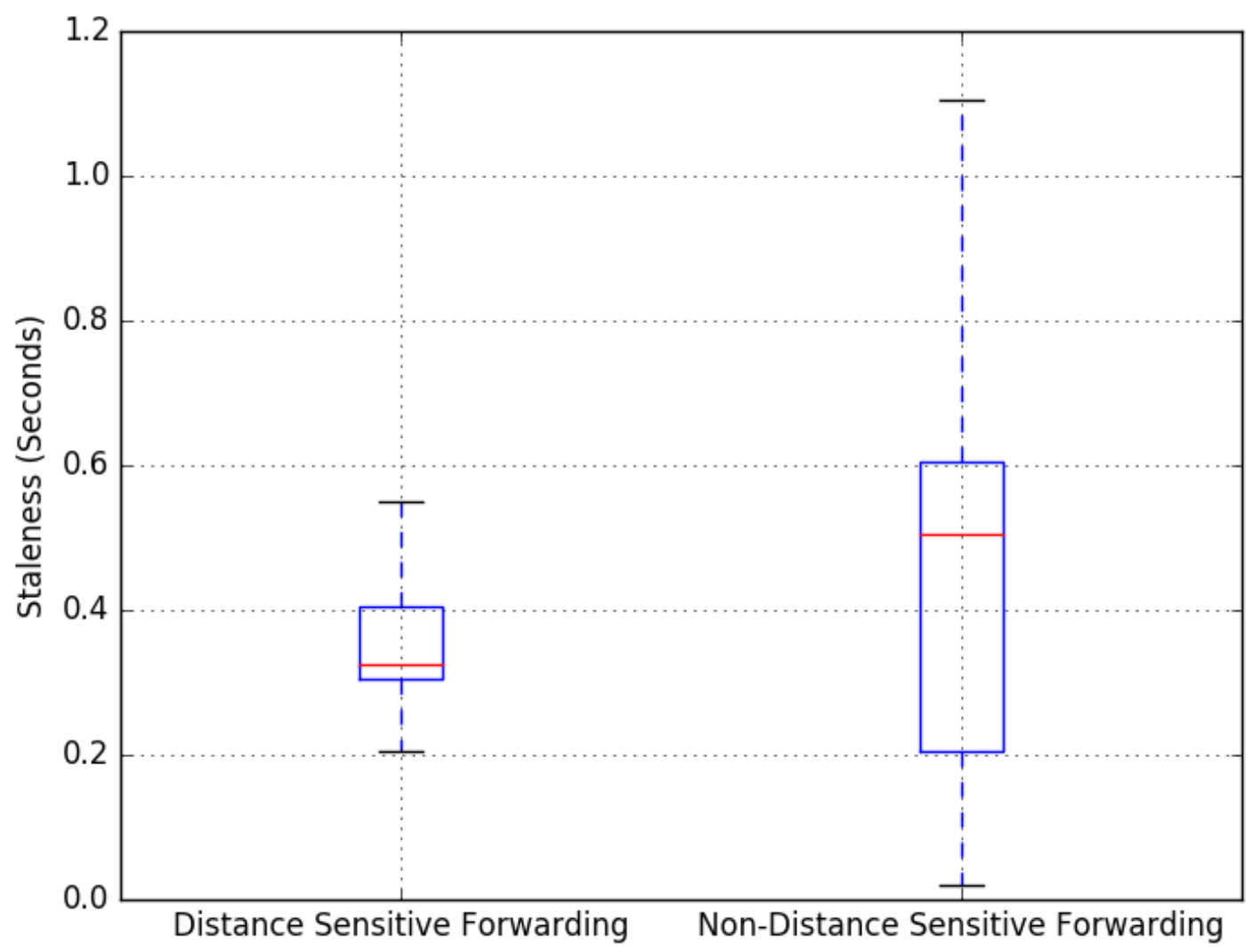

Figure 5.14: Effect of distance sensitive forwarding on staleness of messages within single hop.

\subsubsection{Effect of Aggregation and Forward Packets on Base Trans- mission}

Fig.5.15 shows how staleness of Short range messages $(T x)$ is affected by simultaneously introducing medium range $(F x)$ and Long range $(A g)$ in the network. It can be seen that introducing medium range messages marginally increases the staleness of the single hop messages. Further on addition of long range aggregate messages the staleness in the network 
is maintained.

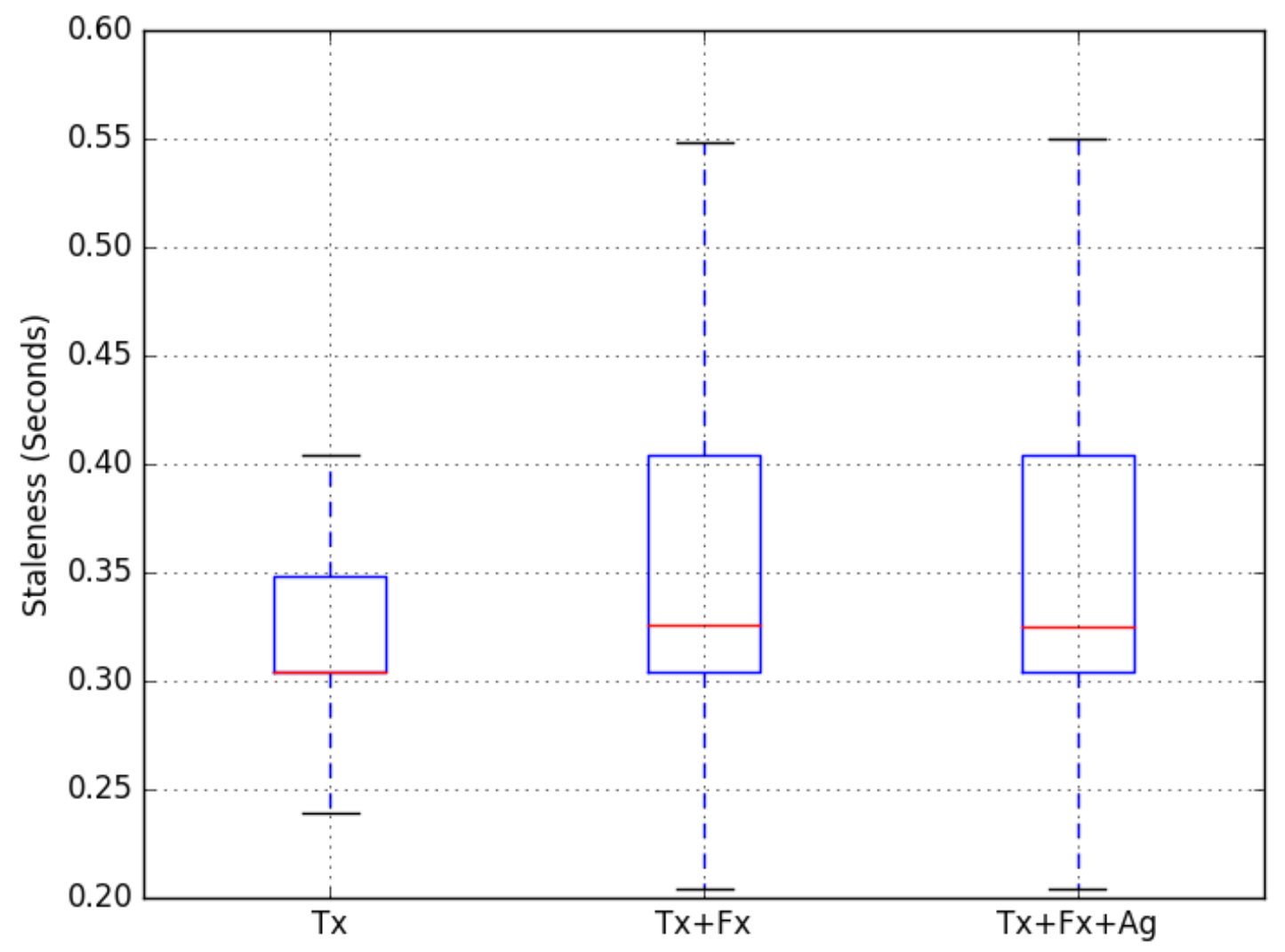

Figure 5.15: Effect of distance sensitive forwarding on staleness of messages within single hop.

\subsubsection{Latency in Aggregate Packets}

The long range aggregate messages should be delivered at very long distances in the shortest time. It can be seen in Fig.5.16 that at very long distance the event messages are delivered within a few seconds. The performance of RoAdNet meets expectations, which is seen by the fact that the staleness of the message within one hop stays unaffected. Higher latency is acceptable at long distances as, vehicle at longer distances have more time to react to an event. Also, the long range information is not safety critical and is meant for rerouting and drive cycle improvement applications. 


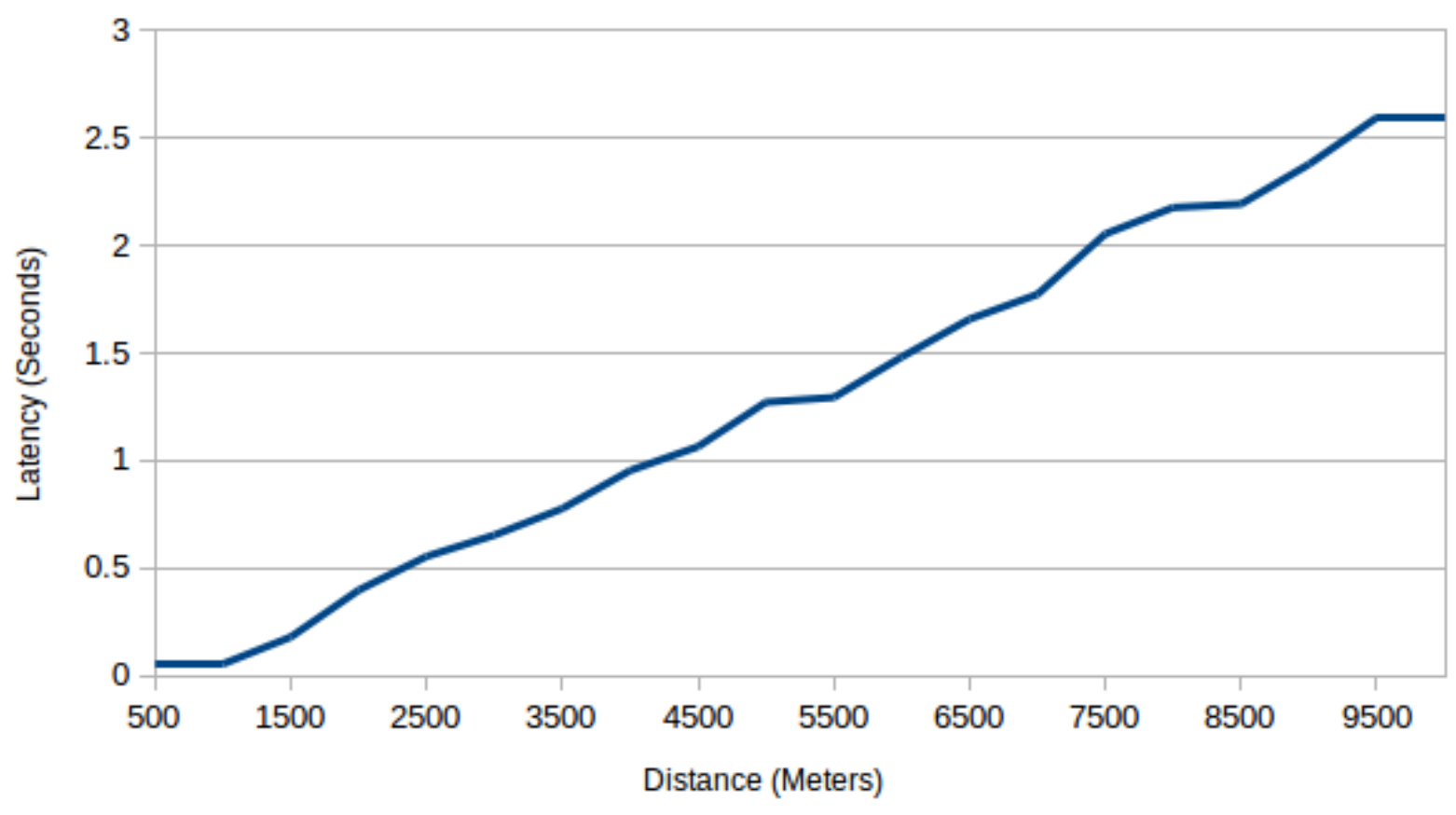

Figure 5.16: Latency of long range messages in RoAdNet

\subsection{A real-world test case for improving efficiency by using prior knowledge of upcoming driving scenar- ios}

In this section we present a real-world scenario to show how prior knowledge of upcoming driving situations can be beneficial in improving vehicle efficiency. This study is based on experiments done with the WVU's EcoCAR3 program. The test cases developed for this study are based on a scenario where an ego vehicle is moving at $30 \mathrm{mph}$ is approaching a stopped/target vehicle. The vehicle is equipped with a Human Machine Interface(HMI) that gives visual feedback to the driver. The HMI displays the distance to the stopped vehicle and suggests the diver to start applying brakes at an appropriate distance.

One of the major applications of RoAdNet is to enable information availability at long range. Availability of such information before the vehicle reaches the region of origin of the message can be used to design energy efficient control algorithms for hybrid vehicles. This experiment is a proof of concept for the belief that, "Availability of prior information can 
be used to improve fuel efficiency of vehicles". Here the information available on the HMI can be treated synonymous to the long range information delivered by RoAdNet.

\subsubsection{Experiment Setup}

The experimental setup has three major components, (1) Ego Vehicle and sensors used, and (2) Hardware and Software architecture of sensors and processors (3) test scenario setup at a test facility.

\section{Ego Vehicle and Sensors}

The West Virginia University's EcoCAR3 Chevy Camaro was used as the 'Ego Vehicle'. The WVU Camaro is a P4 Parallel Hybrid. In a P4 Parallel hybrid the electric motor is mounted post transmission. The Camaro uses E85 ethanol (Flex-Fuel) and Electricity as fuel. The vehicle architecture is given below.

\section{Engine:}

GM 2.4L I4 LEA E85

Peak Power: 136 kW

Peak Torque: $233 \mathrm{Nm}$

Motor:

Parker GVM 210-200S

Peak Intermittent Power: $128 \mathrm{~kW}$

Peak Intermittent Torque: 294 Nm

Peak Continuous Power: $81 \mathrm{~kW}$

Peak Continuous Torque: 195 Nm

ESS: A123 Systems 7x15s2p

Power Output: $40 \mathrm{~kW}$

Peak Power: 118 kW (10 sec)

Energy Storage: $12.6 \mathrm{~kW}-\mathrm{hr}$ 


\section{Hardware and Software Architecture}

The vehicle was equipped with a front facing Bosch radar and a front facing HD camera and two corner radars for recording raw sensor data. For the purpose of this experiment only the front facing radar and camera were used. The data was processed by two processors, a Freescale S32v ADAS Processor and a Nvidia Jetson Tx2. The hardware architecture is shown in Fig.5.17. A 7-inch LCD display driven by a Raspberry Pi processor was used as the Human Machine Interface (HMI) for providing feedback to the driver.

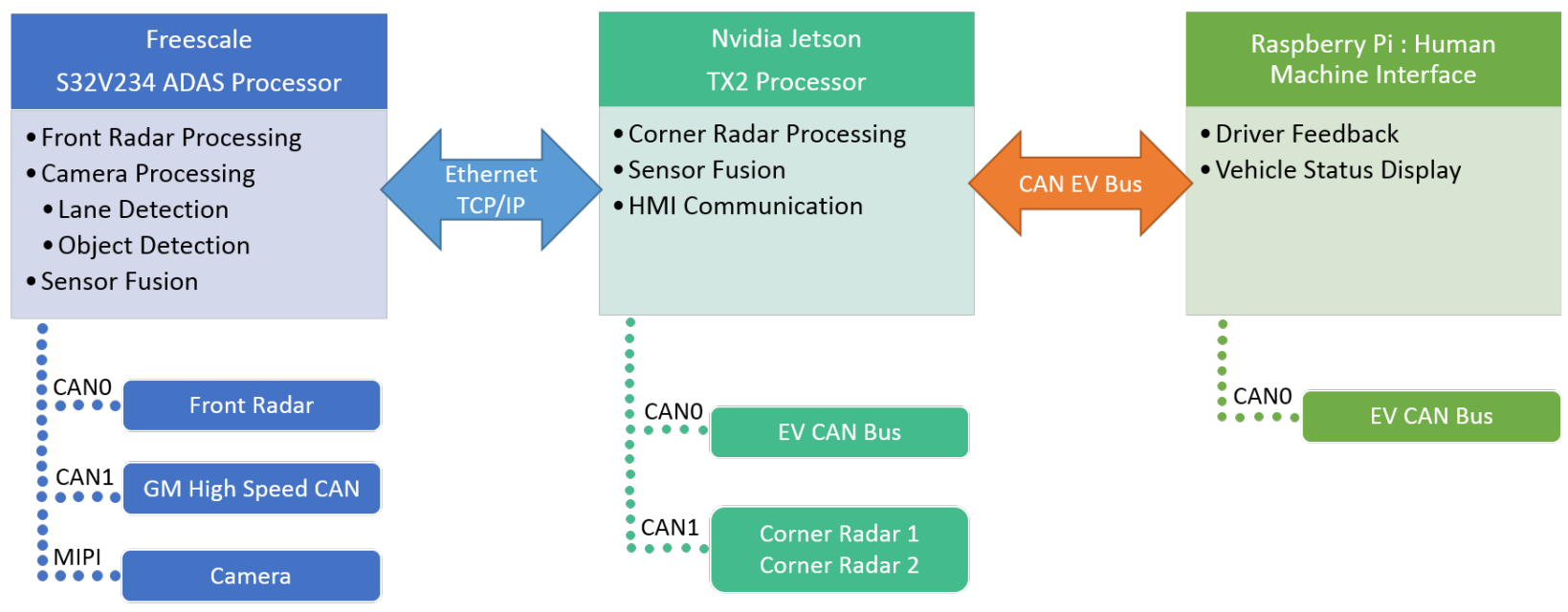

Figure 5.17: EcoCAR Hardware Architecture

The software architecture is show in Fig.5.18. The S32 processor was responsible for reading data from the sensors and the TX2 was used to run a sensor fusion algorithm which would combine the readings from the radar and the camera to give the distance to the targer vehicle in front of th ego vehicle.

The information given by the HMI can be treated as the prior information that the driver received and performed some action based on it. The HMI is shown in Fig.5.19. The HMI was mounted on the front panel of the vehicle to the right of the driver and the stock infotainment system as shown in Fig.5.20. The driver applies brakes when the "Start Braking" button starts to flash. 


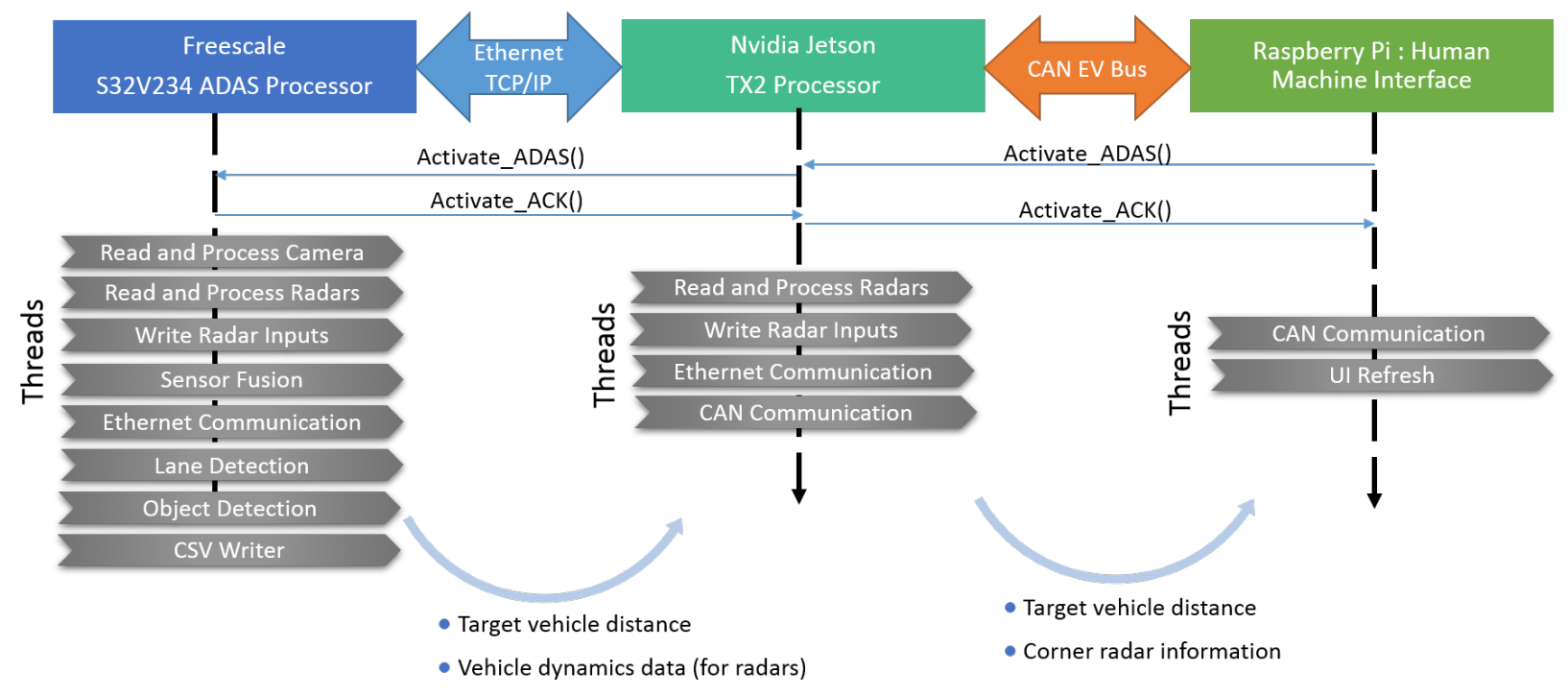

Figure 5.18: EcoCAR Software Architecture

\section{Test Scenario Setup}

The tests were conduction at the University's Band practice field, which is the size of a football field, as shown in Fig.5.21. The target vehicle was parked at one end of the field. Marker cones were laid down at 10 meters gap from the target vehicle. A safety come was placed at 3 meter as a precautionary measure. The Camaro was stationed about 100m behind the target vehicle. For all the three test cases mentioned below, the Camaro accelerated to $30 \mathrm{mph}$, travelling towards the target vehicle and applied brakes at the $10 \mathrm{~m}$ cone for Case 1. Case 1 does not use HMI feedback. For case 2 and 3, brakes were applied when the HMI suggested the user to do so. Manual brakes were applied for case 2 and regenerative braking was used for case 3 .

\subsubsection{Test Cases}

Three test cases were developed for this study:

1. CASE 1: The ego car tips in to reach $30 \mathrm{mph}$ and used mechanical only, hard brake at the $10 \mathrm{~m}$ safety come. This replicates an aggressive, inattentive driver behaviour.

2. CASE 2: The ego car tips in to reach 30mph and brakes, using mechanical brakes when the feedback system says to brake. 


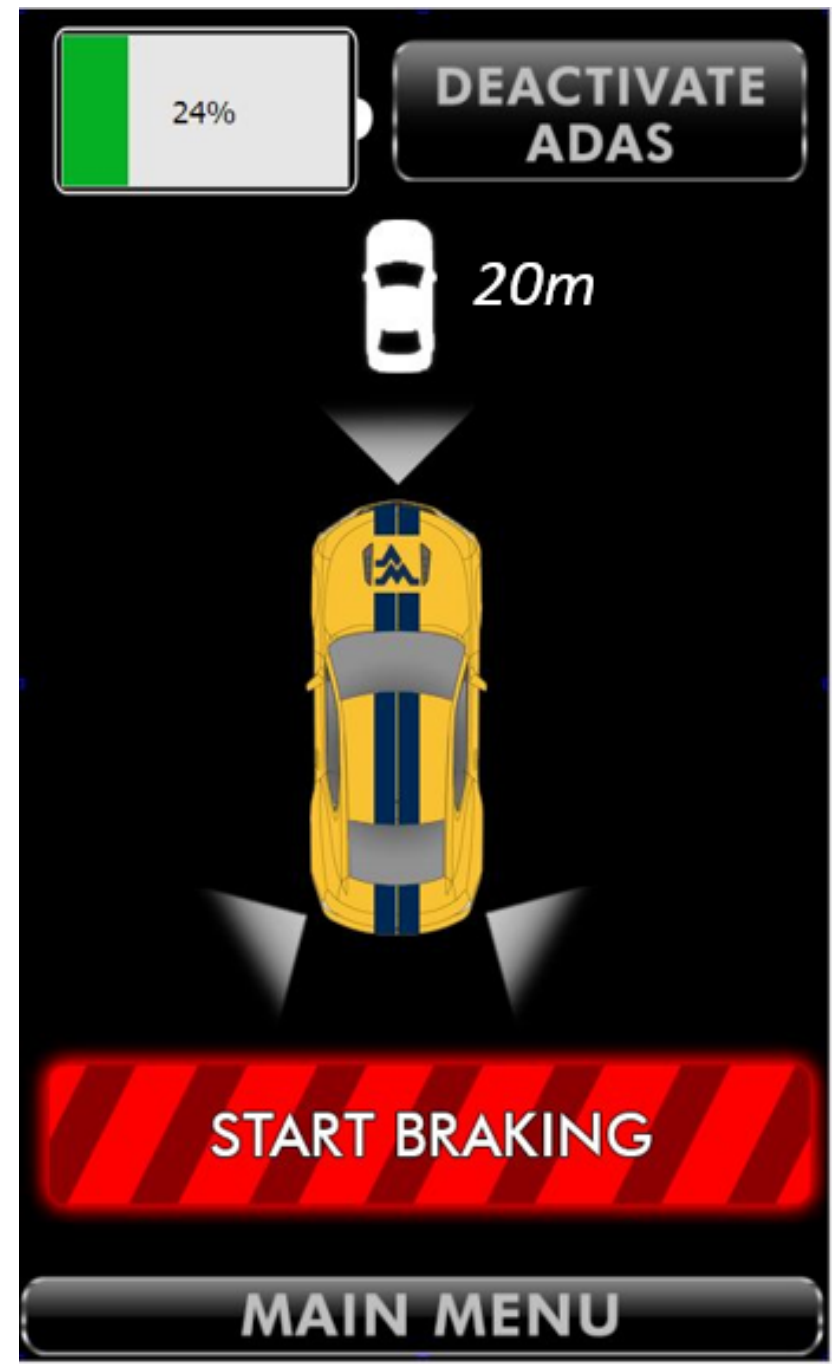

Figure 5.19: EcoCAR HMI Display

3. CASE 3: The ego car tips in to reach 30mph and brakes using ECO-Select mode(Regenerative Braking), when the feedback system says to brake.

Case 1 emulates a distracted driver. It is similar to a scenario where the driver did not see the target vehicle till the very last moment and aggressively brakes to avoid a collision. Case 2 and 3 are scenarios were prior information is available to the driver via the HMI. Each of the test cases were repeated three times and average of the measurements were used for analysis. 


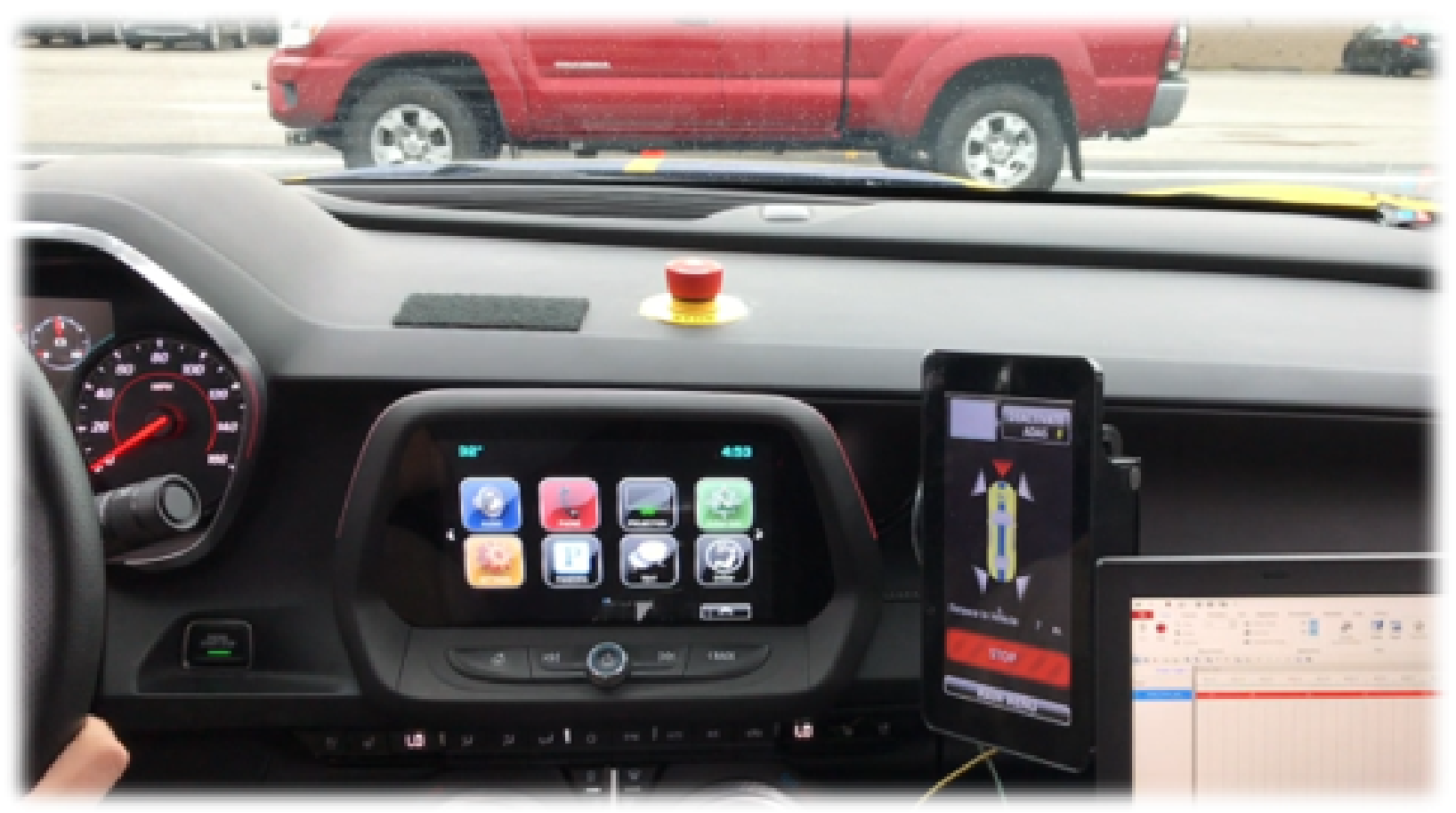

Figure 5.20: EcoCAR HMI mounted in the car

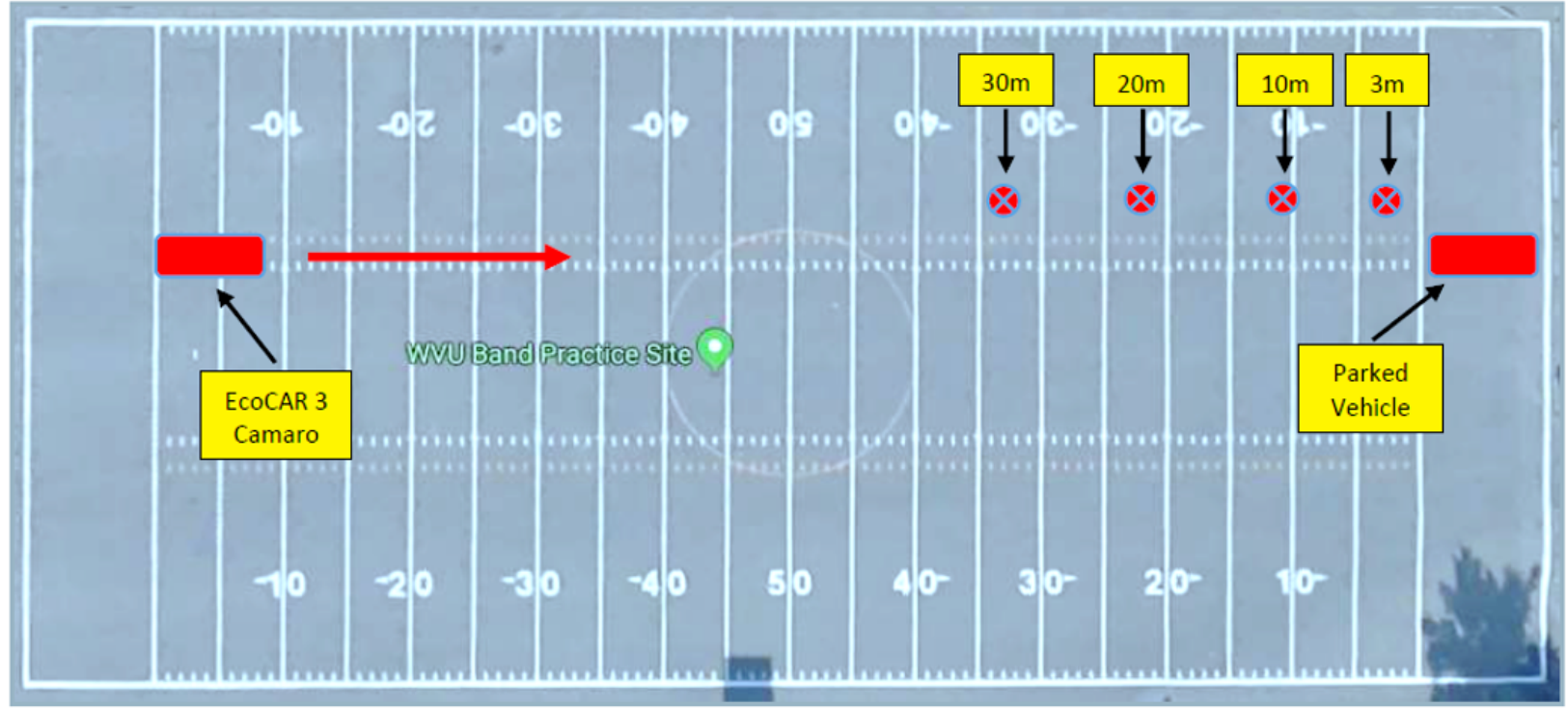

Figure 5.21: The band practice field / Test site

\subsubsection{Results: Regenerative Energy Captured for Different Sce- narios}

The plots in Figure 5.22 and 5.23 show the amount of regenerative energy captured when only mechanical brakes were used and when prior information from the driver feedback 


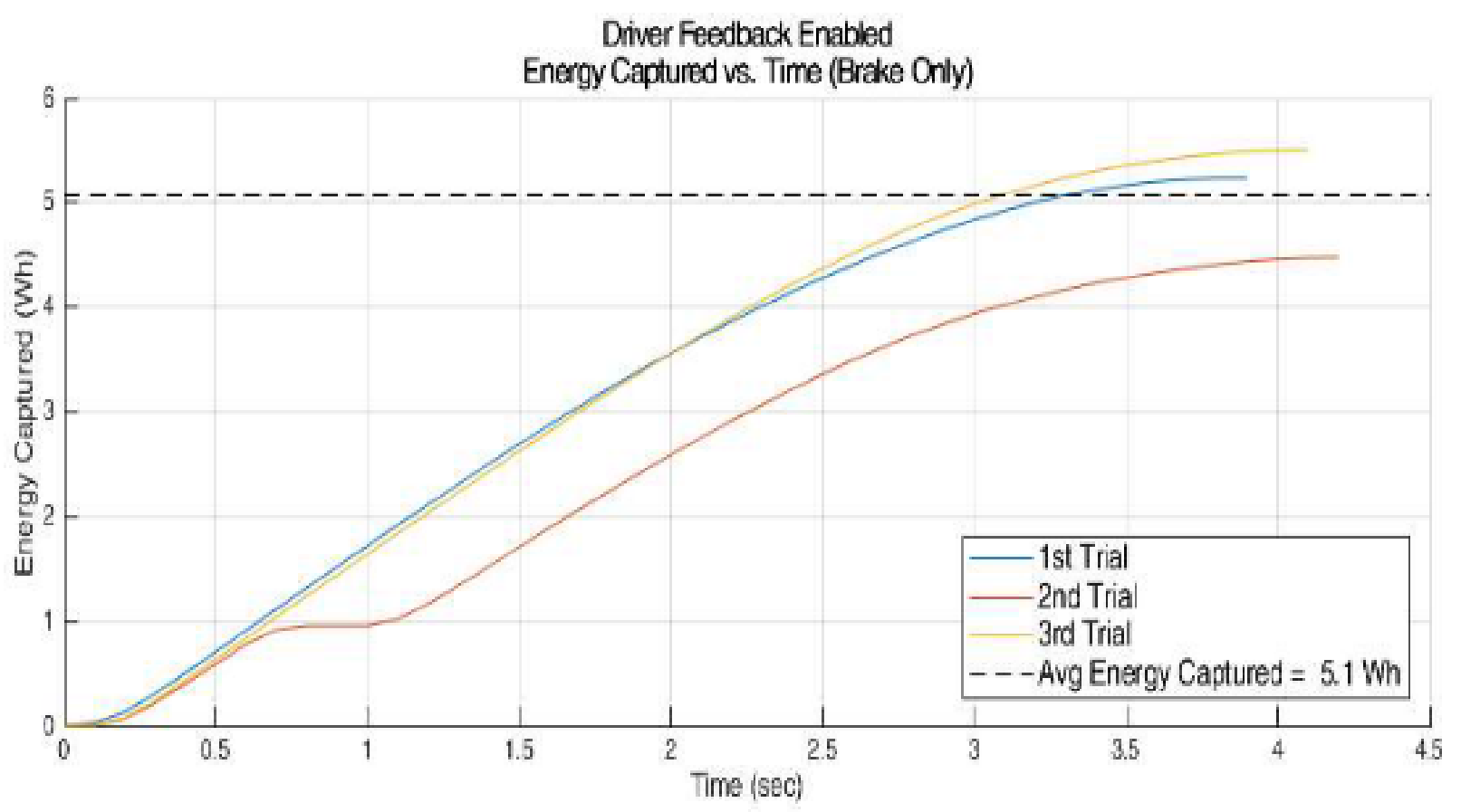

Figure 5.22: Energy captured using mechanical brakes only

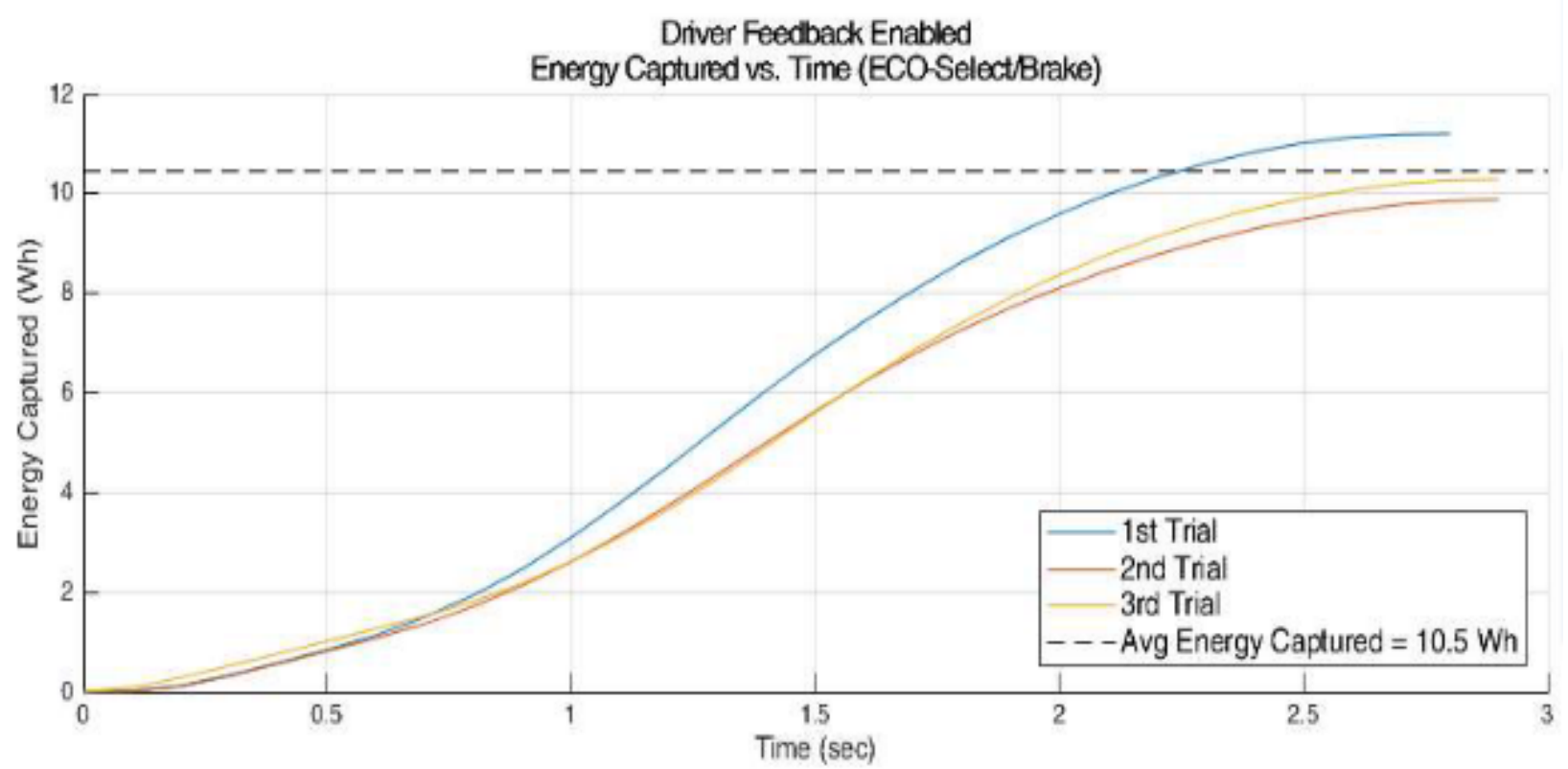

Figure 5.23: Energy captured using generative braking and driver feedback on HMI

system was used to do regenerative braking. Table 5.2 summarizes the results for the various test cases. It can be seen that with prior information about the lead vehicle, the ego vehicle 


\begin{tabular}{|c|c|c|}
\hline $\begin{array}{c}\text { Driver Feedback } \\
\text { Status }\end{array}$ & $\begin{array}{c}\text { Test } \\
\text { Performed }\end{array}$ & $\begin{array}{c}\text { Avg. Total Regen } \\
\text { Energy Captured }\end{array}$ \\
\hline Disabled & $10 \mathrm{~m}$ Hard Brake & 4.0 \\
\hline \multirow{2}{*}{ Enabled } & Brake & 5.1 \\
\cline { 2 - 3 } & Regen + Brake & 10.5 \\
\hline
\end{tabular}

Table 5.2: Average energy captured

captured about twice as much energy using regenerative braking.

\subsubsection{Better fuel efficiency with RoAdNet}

The results obtained from the experiment show that with availability of minimal prior information, we were able to achieve better efficiency in terms of capturing the amount of regenerative energy. Significantly higher efficiency can be expected from a vehicle, especially a hybrid or an electric if better quality of information can be delivered at long distances.

For example, consider a hybrid vehicle driving on a hilly terrain. The torque generating components are an Internal Combustion Engine (ICE) and an Electric Motor (EM) and motors are significantly more efficient than engines. The vehicle's control algorithm uses torque split algorithms to demand torques from ICE and EM to meet the driver's torque demand.

Consider a scenario, where the vehicle is about to climb a hill followed by a long descend. If prior information about the climb and descend is available to the vehicle, it can demand more power from the electric motor as it knows that most of the energy consumed in the climb can be recaptured on the descend by maximizing the regenerative energy captured. The information needed for such an application would come from beyond a single hop communication range.

RoAdNet is capable of providing such information to the vehicle. RoAdNet's medium and long range information can be used to estimate upcoming driving scenarios and run efficient control algorithms to improve the overall efficiency of the vehicle. 


\section{Chapter 6}

\section{Conclusion and Future Work}

\subsection{Conclusion}

In this report, we presented a design and study of multiple factors in the process on building RoAdNet. First, we observed that adding distance sensitivity the forwarding mechanism improves the overall performance of the protocol. The simple approach reduces the bandwidth usage, which results in lesser collisions and better packet delivery ratios. These factors in turn improve staleness and distance estimation error ratio. Then we evaluated the effect of radio range on various network parameters. It was observed that for with increasing radio range, the staleness in the first hop range increased. Longer radio range means more nodes are within the radio range, hence the share of bandwidth available for each node keeps getting smaller. The packet delivery ratio reduced with increase in radio range. After selecting a suitable radio range to aid the distance sensitive forwarding strategy, the effects of some dynamic parameters of RoAdNet were studied.

It was seen that suppression threshold and transmission queue length significantly impact the network performance. We also discussed how ideal combinations of these parameters can be chosen to further improve the performance of RoAdNet.

After understanding the impact of various subsystems and parameters, a multi-resolution transmission strategy was introduced to the protocol. This was the simplest form of RoAdNet. Using this model, we studied the effect of multiple-resolutions. It was seen that by increasing the base transmission frequency and reducing the forwarding frequency, a signif- 
icant improvement occurred across various network parameters. Within single hop range, the staleness of the network decreased and hence the distance estimation improved.

After an extended study of various parameters and subsystems of RoAdNet, its performance as a protocol was evaluated. First it was seen that in Distance Sensitive Forwarding, significantly reduced the staleness of the network. Moreover addition of forwarding and aggregate messages to the network did not significantly affect the staleness of base (single-hop) transmissions.

The studies have shown that RoAdNet can be reliable protocol for addressing multiple concerns in Vehicle to Vehicle Communication. It can be used to transmit short range information for vehicle safety and at the same time send information over long distances to enable efficient rerouting and eco-driving driving features.

\subsection{Future Work}

The work on RoAdNet in this dissertation has established a framework for long range information broadcast in VANET. While RoAdNet is robust and adaptable to dynamic driving conditions, there is scope for improving certain aspects of RoAdNet.

The Randomized Transmission Schedule (RTxS) function uses a random number generator with a Gaussian distribution. Other distribution functions like exponential, log can be tested in the RTxS function to see if there is significant improvement in latency and staleness of messages. The optimization of the RTxS function can help improve the overall latency and staleness of RoAdNet.

RoAdNet has been tested on a simulated highway. It can now be tested in urban scenarios. This will help us understand the effect of highly random traffic movements, constantly changing vehicle speed and cell parameters.

RoAdNet is adaptive to changing cell conditions i.e. average cell speed. It also adapts its beaconing rates by sensing events like accidents and slowdown in the cells. The beaconing can also be made to adapt to changing network conditions. RoAdNet can be programmed to adapt to the density of nodes and messages in the network. 


\section{Appendix A}

\section{List of Abbreviations}

$\begin{array}{ll}\text { AAE } & \text { Aggregate and Adpapt Engine } \\ \text { ADAS } & \text { Advanced Driver Assistance Systems } \\ \text { CAV } & \text { Connected and Automated Vehicles } \\ \text { DEER } & \text { Distance Estimation Error Ratio } \\ \text { DSF } & \text { Distance Sensitive Forwarding } \\ \text { DSRC } & \text { Dedicated Short Range Communication } \\ \text { FE } & \text { Forward Engine } \\ \text { MPS } & \text { Multiple Packet Suppression } \\ \text { PDR } & \text { Packet Delivery Ratio } \\ \text { RE } & \text { Receive Engine } \\ \text { RoAdNet } & \text { Robust Adaptive Networks } \\ \text { RTxS } & \text { Randomized Transmission Schedule } \\ \text { TE } & \text { Transmit Engine } \\ \text { V2V } & \text { Vehicle to Vehicle } \\ \text { V2I } & \text { Vehicel to Infrastructure } \\ \text { V2X } & \text { Vehicle to Everything } \\ \text { VANET } & \text { Vehicular Ad-Hoc Networks }\end{array}$




\section{Appendix B}

\section{List of Symbols}

$\begin{array}{ll}\alpha & \text { Sensitivity factor for distance sensitive forwarding } \\ d_{i j} & \text { Distance between Vehicle } i \text { and } j \\ d & \text { Actual distance to vehicle } \\ \hat{d} & \text { Estimated distance to vehicle } \\ \Delta & \text { Error in distance estimation } \\ \delta_{P} & \text { Distance sensitive delay for packet } P \\ E_{k} & \text { Aggregate Event packet about cell } k \\ \gamma_{k} & \text { Average vehicle speed in cell } k \\ i & \text { Transmitting Vehicle } \\ j & \text { Current Vehicle } \\ k & \text { Cell Id } \\ L(i, j) & \text { Latency of information from Vehicle } i \text { at Vehicle } \mathrm{j} \\ n_{r x} & \text { Total packets received by all vehicles in the network } \\ n_{t x} & \text { Total packets transmitted by all vehicles in the network } \\ Q_{j} & \text { Received messages queue at Vehicle } j \\ P_{i} & \text { Data packet from Vehicle } i \\ \rho & \text { Vehicle density. Vehicles } / 1000 \mathrm{~m} \\ S(i, j) & \text { Staleness of information about vehicle } i \text { at vehicle } j \\ t & \text { Current simulation time } \\ t_{i} & \text { Time when last message was heard from vehicle } i \\ \tau_{l h} & \text { Time since packet was last heard from node } i \\ \zeta_{t x} & \text { Transmission spread (ms) }\end{array}$




\section{References}

[1] Mani Amoozadeh, Hui Deng, Chen-Nee Chuah, H. Michael Zhang, and Dipak Ghosal, "Platoon management with cooperative adaptive cruise control enabled by vanet," Vehicular Communications, vol. 2, no. 2, pp. 110 - 123, 2015.

[2] Vinod Kulathumani Priyashraba Misra, "RoAdNet: A multi-resolution transmission strategy for long range information diffusion in vanets," Vehicular Technology Conference 2019, September 2019.

[3] V. Kulathumani, A. Arora, M. Sridharan, K. Parker, and B. Lemon, "On the repair time scaling wall for manets," IEEE Communications Letters, vol. 20, no. 8, pp. 1623-1626, Aug 2016.

[4] V. Naik, A. Arora, P. Sinha, and H. Zhang, "Sprinkler: A reliable and energy efficient data dissemination service for extreme scale wireless networks of embedded devices," IEEE Transactions on Mobile Computing, vol. 6, no. 7, pp. 777-789, July 2007.

[5] Samuel Madden, Michael J. Franklin, Joseph M. Hellerstein, and Wei Hong, "Tag: A tiny aggregation service for ad-hoc sensor networks," SIGOPS Oper. Syst. Rev., vol. 36, no. SI, pp. 131-146, Dec. 2002.

[6] Omprakash Gnawali, Rodrigo Fonseca, Kyle Jamieson, Maria Kazandjieva, David Moss, and Philip Levis, "CTP: An Efficient, Robust, and Reliable Collection Tree Protocol for Wireless Sensor Networks," ACM Transactions on Sensor Networks (TOSN), vol. 10, no. 3, 2013.

[7] Chalermek Intanagonwiwat, Ramesh Govindan, Deborah Estrin, John Heidemann, and Fabio Silva, "Directed diffusion for wireless sensor networking," IEEE/ACM Trans. Netw., vol. 11, no. 1, pp. 2-16, Feb. 2003.

[8] Samuel Madden, Michael J. Franklin, Joseph M. Hellerstein, and Wei Hong, "Tag: A tiny aggregation service for ad-hoc sensor networks," SIGOPS Oper. Syst. Rev., vol. 36, no. SI, pp. 131-146, Dec. 2002.

[9] Philip Levis, Neil Patel, Scott Shenker, and David Culler, "Trickle: A self-regulating algorithm for code propagation and maintenance in wireless sensor networks," Tech. Rep. UCB/CSD-03-1290, EECS Department, University of California, Berkeley, 2003. 
[10] N. Abramson, "Development of the alohanet," IEEE Transactions on Information Theory, vol. 31, no. 2, pp. 119-123, March 1985.

[11] R. T. B. Ma, V. Misra, and D. Rubenstein, "An analysis of generalized slotted-aloha protocols," IEEE/ACM Transactions on Networking, vol. 17, no. 3, pp. 936-949, June 2009.

[12] Zygmunt J. Haas, Joseph Y. Halpern, and Li Li, "Gossip-based ad hoc routing," IEEE/ACM Trans. Netw., vol. 14, no. 3, pp. 479-491, June 2006.

[13] Roy Friedman, Daniela Gavidia, Luis Rodrigues, Aline Carneiro Viana, and Spyros Voulgaris, "Gossiping on manets: The beauty and the beast," SIGOPS Oper. Syst. Rev., vol. 41, no. 5, pp. 67-74, Oct. 2007.

[14] David Kempe, Jon Kleinberg, and Alan Demers, "Spatial gossip and resource location protocols," J. ACM, vol. 51, no. 6, pp. 943-967, Nov. 2004.

[15] R. Sarkar, X. Zhu, and J. Gao, "Hierarchical spatial gossip for multi-resolution representations in sensor networks," in 2007 6th International Symposium on Information Processing in Sensor Networks, April 2007, pp. 420-429.

[16] Lszl Lovsz, "Random walks on graphs: A survey," 1993.

[17] Uriel Feige, "A tight upper bound on the cover time for random walks on graphs," Random Structures Algorithms, vol. 6, 011995.

[18] Lotker Z Avin C., Kouck M., "How to explore a fast-changing world (cover time of a simple random walk on evolving graphs)," ICALP2008, vol. 5125, 2008.

[19] "Census: Fast, scalable and robust data aggregation in manets," http://arxiv.org/abs/1409.7368.

[20] Nat. Highway Traffic Safety Admin. (NHTSA, "Traffic safety factsresearch note, 2013, usdot," 2018.

[21] T. Kosch, C. Schwingenschlogl, and Li Ai, "Information dissemination in multihop inter-vehicle networks," in Proceedings. The IEEE 5th International Conference on Intelligent Transportation Systems, Sept 2002, pp. 685-690.

[22] C. E. Perkins and E. M. Royer, "Ad-hoc on-demand distance vector routing," in Proceedings WMCSA'99. Second IEEE Workshop on Mobile Computing Systems and Applications, Feb 1999.

[23] David B. Johnson and David A. Maltz, Dynamic Source Routing in Ad Hoc Wireless Networks, pp. 153-181, Springer US, Boston, MA, 1996.

[24] C. A. T. H. Tee and A. Lee, "A novel routing protocol junction based adaptive reactive routing (jarr) for vanet in city environments," in 2010 European Wireless Conference (EW), April 2010, pp. 1-6. 
[25] Brad Karp and H. T. Kung, "Gpsr: Greedy perimeter stateless routing for wireless networks," in Proceedings of the 6th Annual International Conference on Mobile Computing and Networking, New York, NY, USA, 2000, MobiCom '00, pp. 243-254, ACM.

[26] J. Zhao and G. Cao, "Vadd: Vehicle-assisted data delivery in vehicular networks," IEEE Transactions on Vehicular Technology, vol. 57, no. 3, pp. 1910-1922, May 2008.

[27] H. Saleet, R. Langar, K. Naik, R. Boutaba, A. Nayak, and N. Goel, "Intersection-based geographical routing protocol for vanets: A proposal and analysis," IEEE Transactions on Vehicular Technology, vol. 60, no. 9, pp. 4560-4574, Nov 2011.

[28] Z. Cao, K. Shi, Q. Song, and J. Wang, "Analysis of correlation between vehicle density and network congestion in vanets," in 2017 7th IEEE International Conference on Electronics Information and Emergency Communication (ICEIEC), July 2017, pp. 409412.

[29] Yair Allouche and Michael Segal, "Cluster-based beaconing process for vanet," Vehicular Communications, vol. 2, no. 2, pp. 80 - 94, 2015.

[30] Zhihao Zheng, Chengcheng Wang, Pu Wang, Yusha Xiong, Fan Zhang, and Yisheng Lv, "Framework for fusing traffic information from social and physical transportation data," PLOS ONE, vol. 13, no. 8, pp. 1-19, 082018.

[31] Nat. Highway Traffic Safety Admin. (NHTSA, "U.s. department of transportation releases policy on automated vehicle development," 2013.

[32] A. Shaout, D. Colella, and S. Awad, "Advanced driver assistance systems - past, present and future," in 2011 Seventh International Computer Engineering Conference (ICENCO'2011), Dec 2011, pp. 72-82.

[33] Antony Tang and Alice Yip, "Collision avoidance timing analysis of dsrc-based vehicles," Accident Analysis Prevention, vol. 42, no. 1, pp. 182 - 195, 2010.

[34] Y. Liu, P. Watta, B. Jia, and Y. L. Murphey, "Vehicle position and context detection using v2v communication with application to pre-crash detection and warning," in 2016 IEEE Symposium Series on Computational Intelligence (SSCI), Dec 2016, pp. 1-7.

[35] S. Tsugawa, S. Kato, T. Matsui, H. Naganawa, and H. Fujii, "An architecture for cooperative driving of automated vehicles," in ITSC2000. 2000 IEEE Intelligent Transportation Systems. Proceedings (Cat. No.00TH8493), 2000, pp. 422-427.

[36] S. Kato, S. Tsugawa, K. Tokuda, T. Matsui, and H. Fujii, "Vehicle control algorithms for cooperative driving with automated vehicles and intervehicle communications," IEEE Transactions on Intelligent Transportation Systems, vol. 3, no. 3, pp. 155-161, Sep 2002.

[37] Simon Hall and Brahim Chaib-draa, "A collaborative driving system based on multiagent modelling and simulations," Transportation Research Part C: Emerging Technologies, vol. 13, no. 4, pp. 320 - 345, 2005, Agents in Traffic and Transportation: Exploring Autonomy in Logistics, Management, Simulation, and Cooperative Driving. 
[38] S. Tsugawa, S. Jeschke, and S. E. Shladover, "A review of truck platooning projects for energy savings," IEEE Transactions on Intelligent Vehicles, vol. 1, no. 1, pp. 68-77, March 2016.

[39] S. Tsugawa, S. Kato, and K. Aoki, "An automated truck platoon for energy saving," in 2011 IEEE/RSJ International Conference on Intelligent Robots and Systems, Sept 2011, pp. 4109-4114.

[40] C. Wang and H. Nijmeijer, "String stable heterogeneous vehicle platoon using cooperative adaptive cruise control," in 2015 IEEE 18th International Conference on Intelligent Transportation Systems, Sept 2015, pp. 1977-1982.

[41] "String stability and a delay-based spacing policy for vehicle platoons subject to disturbances," https://arxiv.org/abs/1702.01031.

[42] H. C. Righolt and F. G. Rieck, "Energy chain and efficiency in urban traffic for ice and ev," in 2013 World Electric Vehicle Symposium and Exhibition (EVS27), Nov 2013, pp. $1-7$.

[43] H. Kazemi, Y. P. Fallah, A. Nix, and S. Wayne, "Predictive aecms by utilization of intelligent transportation systems for hybrid electric vehicle powertrain control," IEEE Transactions on Intelligent Vehicles, vol. 2, no. 2, pp. 75-84, June 2017.

[44] M. Alsabaan, K. Naik, and T. Khalifa, "Optimization of fuel cost and emissions using v2v communications," IEEE Transactions on Intelligent Transportation Systems, vol. 14, no. 3, pp. 1449-1461, Sept 2013.

[45] G. Naus, R. van den Bleek, J. Ploeg, B. Scheepers, R. van de Molengraft, and M. Steinbuch, "Explicit mpc design and performance evaluation of an acc stop- amp;-go," in 2008 American Control Conference, June 2008, pp. 224-229.

[46] Rajesh Rajamani Jianqiang Wang Shengbo Li, Keqiang Li, "Model predictive multiobjective vehicular adaptive cruise control," in IEEE Transactions on Control Systems Technology, May 2010, pp. 556-566.

[47] R. Muller and G. Nocker, "Intelligent cruise control with fuzzy logic," in Proceedings of the Intelligent Vehicles '92 Symposium, June 1992, pp. 173-178.

[48] et al. Steven E. Shladover, "Effects of cooperative adaptive cruise control on traffic flow: Testing drivers' choices of following distances," Tech. Rep. ISSN 1055-1425, University of California, 2009.

[49] K. J. Malakorn and B. Park, "Assessment of mobility, energy, and environment impacts of intellidrive-based cooperative adaptive cruise control and intelligent traffic signal control," in Proceedings of the 2010 IEEE International Symposium on Sustainable Systems and Technology, May 2010, pp. 1-6.

[50] Richard W. Rothery, Car Following Models. 
[51] M. Treiber and A. Kesting, Traffic Flow Dynamic, 2013.

[52] Louis A. Pipes, "An operational analysis of traffic dynamics," Journal of Applied Physics, vol. 24, no. 3, pp. 274-281, 1953.

[53] P.G. Gipps, "A behavioural car-following model for computer simulation," Transportation Research Part B: Methodological, vol. 15, no. 2, pp. 105 - 111, 1981.

[54] Stefan Krau, "Microscopic modeling of traffic flow : investigation of collision free vehicle dynamics," 1998.

[55] Daniel Krajzewicz, Jakob Erdmann, Michael Behrisch, and Laura Bieker, "Recent development and applications of SUMO - Simulation of Urban MObility," International Journal On Advances in Systems and Measurements, vol. 5, no. 3\&4, pp. 128-138, December 2012. 